\title{
PRECO-D2: Program for Calculating Preequilibrium and Direct Reaction Double Differential Cross Sections
}

\author{
C. Kaibach*
}

\section{DISCLAIMER}

\begin{abstract}
This report was prepared as an account of work smoncorat
Government. Neither the United States Government nored by an agency of the United States bility for the accuracy, warranty, express or implied, or assumes agency thereof, nor any of their process disclosed, or ence herein to any specific conts that its use would not infringe priva, apparatus, product. or - $\quad$ any specific commercial product, process, or service by tely owned rights. Refer.. process, or service by trade name, trademark,
.
\end{abstract}


ABS'TRACT

INTRODUCTION

I. SUMmary OF THE EXCITON MOdel. . . . . . . . . . . . . . . . . . . . 3

I. I Stato Densicies. . . . . . . . . . . . . . . . . . . 3

I. 2 Fesidul [nteraction Rates . . . . . . . . . . . . . . . . 6

I. Particle Erission Ratcs. . . . . . . . . . . . . . . ll

I. 4 Fission Rates. . . . . . . . . . . . . . . . . . . 13

I.5 Closed Form Reactor Equations. . . . . . . . . . . . 14

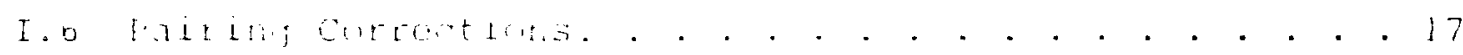

II, DIRECT REACTION MOLELS. . . . . . . . . . . . . . . . . . . . 18

II. 1 Nucieon Transfer . . . . . . . . . . . . . . . . . . . . . 19

II. 2 Knockout and Inelastic Processes with Complex Particle Degrees of Freedom . . . . . . . . . . . . . . . 20

it a . Anglilar distribltions . . . . . . . . . . . . . . . . . . . . . . 23

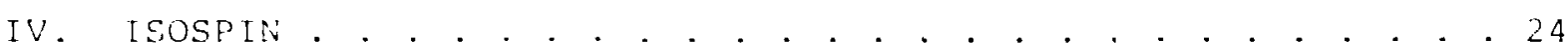

V. DESCRIPTIOR OF TH. PROGRAM. . . . . . . . . . . . . . . . . . . 25

VI. USE OF THE t'ROGRMY. . . . . . . . . . . . . . . . . . . . . . . . . . 28

VI.l Iritial input . . . . . . . . . . . . . . . . . 28

VI. 2 Initial Calculations. . . . . . . . . . . . . . . . 29

VI. 3 Initial Output. . . . . . . . . . . . . . . . . . 30

VI.4 Secondary Input . . . . . . . . . . . . . . . . . . . 32

VI. 5 Secondary Calculations. . . . . . . . . . . . . . . 33

VI. $\overline{0}$ Calculations with Isospin . . . . . . . . . . . . . . . 33

VI.7 Suggested Input . . . . . . . . . . . . . . . . . . 34

REFERENCES . . . . . . . . . . . . . . . . . . . . . . . . . . . . . . 36

FIGURE 1. Flow diagram of PRECO-D2. . . . . . . . . . . . . . . . 38 
TABLE OF CONTENTS (Cont.)

TABLE 1. List nf Variables in EnECO-D2 . . . . . . . . . . . . . 39

TABLE 2. Additional variables in Subroutines . . . . . . . . 44

APPENDIX A. FORTRAN LISTING OF PRECO-D2 . . . . . . . . . . . . . . . . 46

APPENDIX B. SAMPLE INPUT. . . . . . . . . . . . . . . . . . . . 71

AfPendix C. SAMPle output . . . . . . . . . . . . . . . . . . . 72

APPENDIX D. NEW FEATURES IN ERECO-D2. . . . . . . . . . . . . . 107 
PRECO-D2: PRIGEAM FOR CALCLLATING PREEQUILIBRIUM AND DIRECT REACT CON DCLBLE UIFFERENTIAL CROSS SECTIONS

\author{
by: \\ $\therefore$ Fibach
}

\begin{abstract}
The code PRECO-D2 uses the exciton

model for preequilibrium ruclear reactions to describe the emission of particles with mass numbers of $l$ to 4 from an equilibrating composito nucleus. A dis':nction is made between oper and closed configurations in this system and between the multi-step direct (MSD) and multi-step compound (MSC) components of the preequilibrium cross se:tion. Additional MSD components are calculated semi-empirically to account for direct nucleon transfer reactions and direct knockout orocesses involving cluster deyrees of Ereelom. Evaporation from the equiiibrated composite nucleus is ircluded in tile full MSC crose seztion. Output of energy differential and double differentia? cross sections is provided for the Eirst particle emitted frnm the composite system. Multiple particle emission is not considered. This report describes the raction models used in writing PRECO-D2 and explains the organization and utilization of the code.
\end{abstract}




\section{INTROLULTIO:}

PRECO refers to a family of programs of increasing sophistication designed to calculate the energy spectra of particles emitted in nuclear reactions within a statistical model framework which includes non-equilibrium processes. The first series of programs (ending with PRECO-8 aiı PRECOM) ran on a PDP-6 computer with teietype input in the dialogue mode. The present series, whicr began with the code. RECO-A, is suitable for large computing center machines. The model used was first proposed by Griffin [GR66] and is discussed in detail in [KA77] and references therein. The formalism used here has been modicied to include division of the preequiliosium cross section into multi-step direct (MSD) and multi-step compound (MSC) components [KA8I]. Sutroutines have been included to perform sem'-empirical calculations for direct nucleon transfer and for knockout and inelastic processes involving complex particles. Auditional subroutines use the total MSD (including direct) and total MSC (including evaporation) cross sections to calculate the angular distributions for 
the emitted particles. This is done pheno.nenologically $[K A 81 a]$.

\section{SUMMARY OF THE EXCITON MODEL THEORY}

The Griffin or exoiton model is a simple statistical model which totally neglects angular momentum and shell structure. The nucleus is pictured with equally spaced single-particle states, and the interactions responsible for creating and destroying particle/hole pairs are assumed to be two-body, energy conserving and residual in nature. Particle emission rates are calculated from microscopic reversibilit:

\section{I.l State Densities}

The states of the system are enumerated by the number of excited particle, $p$, and hole, $h$, degrees of freedom which they contain. The sum $p+h$ is referred to as the exciton number and is denoted as $\mathrm{n}$. The system is assumed to be formed in a unique particle/hole configuration sfecified by $\left(p_{0}, h_{0}\right)$, and the difference $p-h$ is assumed to remain constant so that $\mathrm{p}-\mathrm{h}=\mathrm{p}_{0}-\mathrm{h}_{0}$. This is not true near equilibrium but is adequate for closed-form preequilibrium calculations. Thus once $p$ is specified for a given class of states, $h$ and $n$ are also specified if the initial 
configuratiou is known. The density of equally spaced single-particle states is denoted as $9_{0}$.

For a system with excitation energy $E$, the overall particle/hole state density is given by

$$
\begin{aligned}
& w(p, h, E)=\frac{g^{n}(p)[E-A(p, h)]^{n-l}}{p ! h !(n-l) !} f(p), \\
& A(p, h)=E_{p a u l i}(p, h)-\left(p^{2}+h^{2}+n\right) / 4 g_{0}, \\
& E_{P a u l i}(p, h)=p_{m}^{2} / g_{0} . \\
& P_{m}=\operatorname{maximum}(p, h) .
\end{aligned}
$$

The quantity $g(p)$ contains corrections to $g_{0}$ assuming that in the real nucleus the single-particle-state density varies as the square root of the energy in the well, with $g_{0}$ representing the average near the Fermi surface. Thus

$$
g(p) \begin{cases}=y_{0}\left[\frac{p}{n}\left(\frac{V+E / n}{v}\right)^{1 / 2}+\frac{h}{n}\left(\frac{V-E / n}{v}\right)^{i / 2}\right] & \text { for } h \leq 2 \\ =g_{0} & \text { for } h>2 .\end{cases}
$$

Here $\mathrm{V}$ is the deptri of the nuclear potential well and is assumed to be $38 \mathrm{MeV}$. The variable $f(p)$ gives corrections to the state density due to the finite depth of the nuclear potential. It has the form (where aiternatives of $V$ may be read in)

$$
E(p) \begin{cases}=1-h\left(\frac{E-V}{V}\right)^{n-1} \theta(E-V)+\frac{h(h-1)}{?}\left(\frac{E-2 V}{V}\right)^{n-1} & \theta(E-V) \\ =1 & \text { for } h<2 \\ & \text { for } h>2 .\end{cases}
$$


Here $\theta$ is the Heaviside Function, rhich is 200 tol it negative argument and unity for a positive one.

In the MSD/MSC formalism, the density of states containing at lecst one unbound particle degree of fre dom is also needed for each class of states. This is referred to simply as the ciensity of unbound atates. In order to specify it, we need to define the effective separation energy, S, which determines the excitation exergy at which particle degrees of Ereedom become unbound. In the present model we assume

$$
S=\operatorname{minimum}\left(\mathrm{B}_{\mathrm{n}}, \mathrm{B}_{\mathrm{p}}+\mathrm{C}_{\mathrm{p}}, \mathrm{B}_{\mathrm{\alpha}}+\mathrm{C}_{\alpha}\right)
$$

where the B's are the bindin: energies and the C's are the Coulomb barriers for the neutron, proton, and alpha particle exit channels. The coulomb barrier for a particle of type $b$ is given in terms of its proton number, $z_{b}$, and the proton umber, $\mathrm{Z}_{\mathrm{B}}$, of the residual nucleus so that

$$
c_{b}=0.75 \frac{z_{b} z_{B}}{A^{1 / 3}} M e V .
$$

The density of unbound states is written as

$$
w^{(u)}(p, h, E)=\frac{g_{p}(p) g_{u}^{n-1}(p)\left[E-A_{1,0}(p, h)-s\right]^{n-1}}{(p-1) ! h !(n-1) !} f_{1}(p) \text {, }
$$

and contains different effective single-particle-state densities, $g_{p}$ and $g_{u}$, for the unbound particle and the remaining degrees of freedom. These are 


$$
\begin{aligned}
& g_{u}(p) \begin{cases}=g_{0}\left[\frac{p-1}{n-1}\left(\frac{v+(E-S) / n}{v}\right)^{1 / 2}+\frac{h}{n-1}\left(\frac{v-(E-S) / n}{v}\right)^{1 / 2}\right] \\
=g_{0} \text { for } h<2\end{cases} \\
& g_{p}(p) \begin{cases}=g_{0}\left(\frac{V+S+(E-S) / n}{V}\right)^{1 / 2} & \text { for } h \leq 2 \\
=g_{0}\left(\frac{V+S}{V}\right)^{1 / 2} & \text { for } h>2 .\end{cases}
\end{aligned}
$$

The finite well depth corrections are contained in the quantity $f_{1}(p)$ which, for $h \subseteq 2$, is given by

$$
\begin{aligned}
& E_{l}(p)=\frac{1}{p} \sum_{i=1}^{k} \sum_{j=0}^{h}(-1)^{i+i+1}\left(\begin{array}{l}
p \\
i
\end{array}\right)\left(\begin{array}{l}
h \\
j
\end{array}\right)\left(\frac{E-i S-j V}{E-S}\right)^{n-1} \\
& \theta(E-i S-j V)
\end{aligned}
$$

while for h>2 only $j=0$ terms are included. The quantity $A_{1,0}(p, h)$ is analogous to $A(p, h)$ and is defined by [KA83]

$$
A_{i, 0}(p, h)=\frac{p_{m}^{2}+\left(p_{m}-i\right)^{2}}{2 g_{0}}-\frac{(p-i)^{2}+h^{2}+n-i}{4 g_{0}} \text {. }
$$

The density of bound states is also needed and is found by a simple difference

$$
\omega^{(b)}(p, h, E)=\omega(p, h, E)-\omega^{(u)}(p, h, E) .
$$

\section{I.2 Residual Interaction Rates}

The residual interactions which are responsible for changing the exciton number of the system are assumed to be energy conserving and two-body in nature so that allowed transitions are those for which $\Delta p=\Delta h=0, \pm 1$. The rates for 
these three yeneral categories of transitions are denoted $1_{1}, i_{+}$and $i_{-}$. In adultion, the superscispts $u$ ard b are used to indicate the unbound or bound character of the initial and Final state in the interaction. Thus $\lambda_{+}^{(u b)}(p, h, E)$ is the average rate for creating a particle, hole pair starting from an unbound state in the class specified by $(p, h)$ and going to a bousd final state in the class $(p+1, h+1)$. Ail of the transition rates have the general form exemplified below:

$$
\lambda_{+}^{(u b)}(p, h, E)=(2 \pi / h) M^{2}(p) \omega_{+}^{(u b)}(p, h, E),
$$

where $M^{2}$ is the mean square two-body matrix element and $\omega_{+}^{(u b)}$ gives the density of accessible final states. The quantity $\mathrm{M}^{2}$ is given by [KA78]

$$
M^{2}(p) \begin{cases}=\frac{k}{A^{3}} \frac{n}{E}\left(\frac{E / n}{7 M e V} \frac{E / n}{2 M e v}\right)^{1 / 2} & \text { for } \quad E / n<2 M e V \\ =\frac{k}{n^{3}} \frac{n}{E}\left(\frac{E / n}{7 M e V}\right)^{1 / 2} & \text { for } 2 M e V \leq E / n<7 M e V \\ =\frac{k}{A^{3}} \frac{n}{E} & \text { for } 7 \mathrm{MeV} \leq E / n \leq 15 \mathrm{MeV} \\ =\frac{k}{A^{3}}-\frac{n}{E}\left(\frac{15 \mathrm{MeV}}{E / n}\right)^{1 / 2} & \text { for } 15 \mathrm{MeV}<E / n\end{cases}
$$

where $A$ is the mass number $O F$ the composite nucleus and $k$ is an empirical constant usiullv taken to be $135 \mathrm{MeV}^{3}$.

Several quantities are useful in describing the densities of accessible final states. We thorefore define

$$
x_{i}(p)=\left[E-A_{i, 0}(p, h)-i S\right] \theta\left(E-A_{i, 0}(p, h)-i S\right),
$$


as well as the correction functions

$$
\begin{aligned}
& f_{+}(P)\left\{\begin{array}{rr}
=1-\left(\frac{E-S-V}{E-S}\right)^{n-1} \theta(E-S-V)-\frac{1}{2}\left(\frac{E-2 S}{E-S}\right)^{n-1} \theta(E-2 S) \\
+\frac{1}{2}\left(\frac{E-2 S-V}{E-S}\right)^{n-1} \theta(E-2 S-V) & \text { for } h \leq 2, \\
=1-\frac{1}{2}\left(\frac{E-2 S}{E-S}\right)^{1 !-1} \theta(E-2 S) & \text { for } h>2,
\end{array}\right. \\
& E_{0}(p)=1-\frac{1}{2}\left(\frac{E-2 S}{E-S}\right)^{n-1} \theta(E-2 S) ;
\end{aligned}
$$

and the effective single-particle-state densities

$$
\begin{array}{ll}
g_{a}(p+1) \begin{cases}=\frac{n-1}{n} g_{u}(p+l)+\frac{1}{n} g_{p}(p+l) & \text { for } h<1, \\
=g_{0} & \text { for } h>1\end{cases} \\
g_{h}=g_{0}[(v-S) / v]^{1 / 2}, &
\end{array}
$$

where we have assumed that $V>S$. The fraction of unbound states in a given class which have more than one unbound particle also enters in and is given by

$$
m(p)=\frac{\sum_{i=2}^{p} \sum_{j=0}^{h}(-1)^{i+j}\left(\begin{array}{l}
p \\
i
\end{array}\right)\left(\begin{array}{l}
h \\
j
\end{array}\right) \theta(E-i S-j V)(E-i S-j V)^{n-1}}{\sum_{i=1}^{p} \sum_{j=0}^{h}(-1)^{i+j+1}\left(\begin{array}{l}
p \\
i
\end{array}\right)\left(\begin{array}{l}
h \\
j
\end{array}\right) \theta(E-i S-j V)(E-i S-j V)^{n-1}}
$$

for $h \leq 2$, while for $h>2$ only $j=0$ is considered. Finally we need the three miscellaneous quantities

$$
E_{u}(p)=\frac{2 f_{1}(p) m(p)}{p-1}\left(\frac{E-S}{E-2 S}\right)^{n-1},
$$


$G_{1}=\operatorname{maximum}(E-V, 0)$,

$G_{2}=\operatorname{maximum}(E-V-S, 0)$.

In terms of these quantities, the necessary densitier of final states for the two-body interactions are given by

$$
\begin{gathered}
w_{+}^{(u u)}(p, h, E)=\frac{g_{a}(p+1) g_{1}^{2}(p+1)}{2 n} \frac{x_{1}^{n+1}(p+1)\left(\frac{1}{n} f+(p+1)+\frac{n-1}{n} r(p+1)\right)}{x_{1}^{n-1}(p)} \\
+\frac{m(p)}{1-m(p)} \omega_{+}^{(u b)}(p, n, E), \quad(25)
\end{gathered}
$$

$w_{+}^{(U b)}(p, h, E)=\frac{1-m(p !}{2 n(n+1)} \mid g^{3}(p+1) f(p+1)$

$$
\begin{aligned}
\left\{n^{2}\left[x_{n}(p+1)-x_{1}(p)\right]^{2}\right. & \left.+\frac{n}{2}\left[x_{0}^{2}(p+1)-x_{1}^{2}(p)\right]+x_{0}(p+1) x_{1}(p)\right\} \\
& \left.-2 g_{p}(p+1) g_{u}^{2}(p+1) E_{+}(p+1) \frac{x_{1}^{n+1}(p+1)}{x_{1}^{n-1}(p)}\right\},(26)
\end{aligned}
$$

$$
\begin{aligned}
& w_{+}^{(b u)}(p, h, E)=\frac{g_{p}(p+1)}{x_{0}^{n-1}(p) f(p)}-\frac{g_{u}^{2}(p+1) h}{p x_{1}^{n-1}(p) f_{1}(p)} \\
& \quad\left\{\frac{x_{1}^{n+1}(p+1)-p x_{2}^{n+1}(p+1)}{2 n(n+1)}-\frac{1}{4 n(n+1)}\right. \\
&\left\{G_{1}^{n-1}\left[n(n-1) G_{1}^{2}-2(n+1)(n-1) G_{1} x_{1}(p+1)+n(n+1) x_{1}^{2}(p+1)\right]\right. \\
&\left.\left.-p G_{2}^{n-1}\left[n(n-1) G_{2}^{2}-2(n+1)(n-1) G_{2} x_{2}(p+1)+n(n+1) x_{2}^{2}(p+1)\right]\right\}\right\},
\end{aligned}
$$




$$
\begin{aligned}
w_{+}^{(b, b)}(p, h, E)= & \frac{1}{2 n(n+1)} \frac{1}{x_{0}^{n-1}(p) F(p)-p x_{1}^{n-1}(p) f_{1}(p)} \\
& \left\{n g^{3}(p+1) x_{0}^{n+1}(p+1) f(p+1)\right. \\
& -p(n-1) g_{u}^{3}(p+1) x_{1}^{n+1}(p+1) f_{1}(p+1) \\
& -p g^{3}(p+1) x_{1}^{n-1}(p) f_{1}(p) \\
& \left.\left\{\frac{n^{2}}{2}\left[x_{0}(p+1)-x_{1}(p)\right]^{2}+\frac{n}{2}\left[x_{0}^{2}(p+1)-x_{1}^{2}(p)\right]+x_{0}(p+1) x_{1}(p)\right\}\right) \\
& -w_{+}^{(b u)}(p, h, E),
\end{aligned}
$$

$$
\begin{aligned}
\omega_{0}^{(u b)}(p, h, E) & =\frac{[1-m(p)] g_{0}^{2}}{n}\left(\frac{p+2 h-1}{2} n\left[x_{0}(p)-x_{1}(p)\right] f(p)\right. \\
& \left.+(p-1) x_{1}(p)\left[f(p)-2 f_{0}(p)\right]\right)
\end{aligned}
$$

$$
\begin{aligned}
& w_{0}^{(b u)}(p, h, E)=\frac{g_{u}(p) g_{p}(p)}{2 n} \frac{1}{x_{0}^{n-1}(p) f(p)-p x_{1}^{n-1}(p) f_{1}(p)} \\
& \left((p+2 h-1)\left\{2 x_{1}^{n}(p)+n\left[x_{0}(p)-x_{1}(p)\right] x_{1}^{n-1}(p)\right\} f(p)\right. \\
& -(p-1)(p+2 h-1)\left\{2 x_{2}^{n}(p)+n\left[x_{1}(p)-x_{2}(p)\right] x_{2}^{n-1}(p)\right\} f_{1}(p) \\
& \left.-4(n-1) x_{1}^{n}(p) f_{1}(p)+4(p-1)(n-2) x_{2}^{n}(p) f_{2}(p)\right)
\end{aligned}
$$$$
\omega_{-}^{(u b)}(p, h, E)=[1-m(p)] g_{h} h(h-1) / 2,
$$ 


$$
\begin{aligned}
& \omega_{-}^{(b u)}(p, h, E)=\frac{g_{p}(p-1) h p(p-1)}{4} \frac{1}{x_{0}^{n-1}(p) E(p)-p x_{1}^{n-1}(p) f_{1}(p)} \\
& \quad\left\{x _ { 1 } ^ { n - 3 } ( p - 1 ) \left[(n-2)(n-3) x_{1}^{2}(p-1)\right.\right. \\
& \left.\quad-2(n-1)(n-3) x_{1}(p-1) x_{0}(p)+(n-1)(n-2) x_{0}^{2}(p)\right] E_{(p)} \\
& -\quad(p-2) x_{2}^{n-3}(p-1)\left[(n-2)(n-3) x_{2}^{2}(p-1)\right. \\
& \left.\quad-2(n-1)(n-3) x_{2}(p-1) x_{1}(p)+(n-1)(n-2) x_{1}^{2}(p)\right] f_{1}(p) \\
& \left.-4\left[x_{1}^{n-1}(p) E,(p)-(p-2) x_{2}^{n-1}(p) E_{u}(p)\right]\right\} .
\end{aligned}
$$

I. 3 Particle Emission Rates

The average rate for emitting a particle of type $b$ and energy $\varepsilon$ from an unbound state specified by $(\mathrm{p}, \mathrm{h})$ is derived from microscopic reversibility to be

$W_{b}^{(u)}(p, h, E) d E=\frac{\left(2 s_{b}+1\right)}{\pi^{2} \hbar^{3}} A_{b} \varepsilon \sigma_{b}(E) d \varepsilon Q_{b}(p) \frac{\omega\left(p-A_{b}, h, U\right)}{\omega^{(u)}(p, h, E)}$,

where $s_{b}$ and $A_{b}$ are the spin and mass numbers of the emitted particle. The quantity $\sigma_{b}$ is the cross section for the inverse process of composite nucleus formation, while U is the excitation energy of the residual nucleus. The quantity $Q_{b}(p)$ takes account of the fact that proton and neutron degrees of freedom are distinguishable. It. assumes that in each pair-creation interaction protons and neutrons 
are excited with the relative Erobabilities $\mathrm{Z} / \mathrm{A}$ and $\mathrm{N} / \mathrm{A}$, and it has the form

$$
Q_{b}(p) \begin{cases}\frac{\left(p-A_{b}\right) !}{p !} \sum_{i=0} \frac{\left(p-A_{a}\right) !}{i !\left(p-A_{a}-i\right) !}\left(\frac{Z_{T}}{A_{T}}\right)^{i-z_{b}}\left(\frac{N_{T}}{A_{T}}\right)^{p-A_{a}-i-N_{b}} \\ \frac{\left(z_{a}+i\right) !}{\left(z_{a}+i-z_{b}\right) !} \frac{\left(p-z_{a}-i\right) !}{\left(p-z_{a}-i-N_{b}\right) !} & \text { for } n \leq \bar{n} \text { and } n \leq 20 \\ =1 & \text { for } n>\bar{n} \text { or } n>20 .\end{cases}
$$

Here $\mathrm{z}_{b}$ and $\mathrm{N}_{\mathrm{b}}$ are the proton and reutron numbers of the emitted particle, and $A_{a}=z_{a}+N a$ is the nucleon number of the projectile. The quantities $\mathrm{Z}_{\mathrm{T}}{ } \mathrm{N}_{\mathrm{T}}$ and $\mathrm{A}_{\mathrm{T}}$ are the proton, neutron and mass numbers of the target. Finaliy $\vec{n}$ is the most probable number of degrees of freedom at equilibrium (i.e., the number for which $\omega(p, h, E)$ is a maximum).

An alternative to $Q_{b}(p)$ has been added in this newer version of PRECO-D. It is assumed that proton and neutron particle/hole pairs are excited in proportion to the state densities of the configurations formed [GA77]. This assumption is more consistent with the assumption made in deriving the emission rates from microscopic reversibility $(\because .$, all configurations of a given $\mathrm{p}, \mathrm{h}$, and $\mathrm{E}$ are equally likely to be populated). It leads to the $Q_{b}$ values of 


$$
\begin{aligned}
& (z) \therefore-\because b, N i^{n,-n !} \quad\left(p-A_{n}\right) ! \quad h !
\end{aligned}
$$

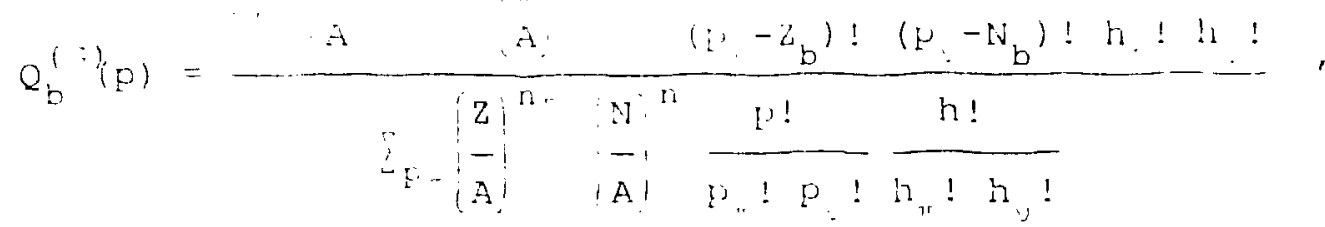

where the subscripts $;$ and rofer to proton and neutron degrees of freedom, and $Z, N$, and $A$ are for the composite nucleus. Either fom for o may be chosun by suacifyina an input parumeter.

Particle amission ratis for the bound states aro, of course, zero.

At equilibrium the emission rates for the full system are calculated from the Weisskopf-Ewing evaporation formula and are given by

$$
W_{b}(E) d \varepsilon=\frac{\left(2 s_{b}+1\right)}{\pi^{2} \hbar^{3}} A_{b} E \sigma_{b}(\varepsilon) d r-\frac{w(U)}{\omega(E)} \text {, }
$$

where in the present case the state densities are assumed to be those for a single Fermi gas,

$$
\omega(E) \approx E^{-1} \exp \left[2(a E)^{1 / 2}\right] \text {, }
$$

and $\mathrm{a}=\pi^{2} g_{0} / 6$.

\section{4 Eission Rates}

In order to facilitatc application of the model to ver: heavy nuclei, fission competition in the equilibrium limit has been considered. Barrier penetration has been neglected 
in order to obtain a closed-form integral over the kinetic energy degree of freedom. Thus one obtains

$$
V_{E}(E)=\frac{1}{2 \pi h} \int_{0}^{E-E_{L}} \frac{\omega\left(E-B_{F}-\varepsilon_{E}\right)}{\omega(E)} d \varepsilon_{f},
$$

where $\varepsilon_{i}$ is the kinetic energy in the fission degree of freedom.

I.5 Closed-Form Reaction Equations

In the closed-form ajproach, the strength of the system is imagined to pass sequentially through configurations of increasing complexity until the most probable class of states at equilibrium (specified by $\bar{n}=\bar{p}+\bar{h}$ ) is reached. Particle emission is allowed at each stage.

In the MSD/MSC formalism it is necessary to know for each class of states how much of the reaction strength passes through unbourd states and how much through bound states. These quantities are denoted $S_{u}(p, h)$ and $S_{b}(p, h)$, respectively. In addition, it is necessary to know the amount of strength which passes through unbound states of the class $(p, h)$ and which was unbound at all previous stages as well. This is denoted $S_{d}(p, h)$ and is the strength responsible for the MSD cross section.

Strengths of the three types arriving at the $(p, h)$ stage of the hierarchy through pair creation are denoted $P_{u}(p, h)$, $P_{b}(p, h)$ and $P_{d}(p, h)$, respectively, and differ from the 
corresponding $S$ iuantities by the offlot of the " and interactions.

Initially it is assumed that thi unbound states are populated in proportion to their relative state densities so that

$$
\begin{aligned}
& P_{u}\left(P_{0}, h_{0}\right)=P_{d}\left(P_{0}, h_{0}\right)=w^{(1)}\left(P_{0}, h_{0}, E\right) / w\left(P_{0}, h_{0}, E\right), \\
& P_{b}\left(P_{0}, h_{0}\right)=1-P_{u}\left(E_{0}, h_{0}\right) .
\end{aligned}
$$

At this exciton number and at each succeeding exciton number it is assumed that there will be at most one excitonscattering interaction which takes the system from a bound to an unbound configuration or lick "esa. Further, it is as jumed that there can be at most one pair-annihilation interactinn whish shanges the bound;unbound character of the state and tiat it will immediately be followed by a paircreation interaction which does not change the bound/anbound character. Thus in effect $\lambda_{0}^{(u b)}$ and $\lambda_{-}^{(u b)}$ are treated together, and similarly for $\lambda_{0}^{(b u)}$ and $\lambda_{-}^{(b u)}$.

Because of these assumptions, strength arriving for the first time at a given hierarchy is assigned a lifetime of

$$
\begin{aligned}
\mathrm{T}_{u}(p, E)=\left[\lambda_{+}^{(u)}(\mathrm{p})+\lambda_{+}^{(u b)}(p)\right. & +\lambda_{0}^{(u b)}(p)+\lambda_{-}^{(u b)}(p) \\
& \left.+\sum_{b} \int w_{b}(p, E) d \varepsilon\right]^{-1}
\end{aligned}
$$

(where the hole label has been suppressed) if i'. is in unbound states or

$$
T_{b}(p, E)=\left[\lambda_{+}^{(b b)}(p)+\lambda_{+}^{(b u)}(p)+\lambda_{0}^{(b u)}(p)+\lambda_{-}^{(b u)}(p)\right]^{-1}
$$

if it is in bound configurations. Strength which has already 
undergone an exciton-scattering or pair-annihilation interaction starting from this hierarchy is assigned a different lifetime, one of the two quantities

$$
\begin{aligned}
& T_{u}^{\prime}(p, E)=\left[\lambda_{+}^{(u u)}(p)+\lambda_{+}^{(u b)}(p)+\sum_{b} \int w_{b}(p, E) d \varepsilon\right]^{-1}, \\
& T_{b}^{\prime}(p, E)=\left[\lambda_{+}^{(b b)}(p)+\lambda_{+}^{(b u)}(p)\right]^{-1}
\end{aligned}
$$

The strengths passing through states of different $p$ values are then found from a set of recursion relations:

$$
\begin{aligned}
& S_{d}(p)=P_{d}(p), \\
& S_{u}(p)=P_{u}(p)+P_{b}(p) \Gamma_{u}^{(b u)}(p) \Gamma_{u}^{\prime}(p) / T_{u}(p), \\
& S_{b}(p)=P_{b}(p)+P_{u}(p) \Gamma_{0}^{(u b)}(p) T_{b}^{\prime}(p) / T_{b}(p),
\end{aligned}
$$

and

$$
\begin{aligned}
& P_{d}(p+1)=s_{d}(p) \Gamma_{+}^{(u u)}(p) \equiv P_{d}(p) \Gamma_{+}^{(u u)}(p), \\
& P_{u}(p+1)=S_{u}(p) \Gamma_{+}^{(u u)}(p)+S_{b}(p) \Gamma_{+}^{(b u)}(p), \\
& P_{b}(p+l)=s_{b}(p) \Gamma_{+}^{(b b)}(p)+s_{u}(p) \Gamma_{+}^{(u b)}(p),
\end{aligned}
$$

with

$$
\begin{aligned}
& \Gamma_{+}^{(u u)}(p)=\lambda_{+}^{(1, u)}(p) T_{u}(p), \\
& \Gamma_{+}^{(u b)}(p)=\lambda_{+}^{(u b)}(p) T_{u}(p), \\
& \Gamma_{0}^{(u b)}(p)=\left[\lambda_{0}^{(1, b)}(p)+\lambda_{-}^{(u b)}(p)\right] \mathbb{T}_{u}(p),
\end{aligned}
$$

and similarly for $\Gamma_{+}^{(b b)}, \Gamma_{+}^{(b u)}$ and $\Gamma_{0}^{(b u)}$ but with the $u$ and b labels interchanged.

The preequilibrium energy differential cross sections are then given in terms of the strength variables. The total MSD and MSC preequilibrium cross sections are 


$$
\begin{aligned}
& \frac{d a}{d \bar{c}}(a, b)_{P R E}=J_{a}\left(\varepsilon_{d}\right) \sum_{\mathrm{i}}^{\bar{p}} \bar{b}_{u} S_{u}(p) \mathrm{T}_{u}(p) W_{\mathrm{b}}^{(u)}(p, \varepsilon), \\
& \frac{\mathrm{d} \sigma}{\mathrm{d} E}(\mathrm{a}, \mathrm{b})_{\mathrm{H} \cdot \mathrm{re}-M S D}=\sigma_{a}\left(\varepsilon_{a}\right) \sum_{\mathrm{p}=E_{0}}^{\bar{p}} S_{\mathrm{d}}(p) \mathrm{T}_{u}(p) \mathrm{w}_{\mathrm{b}}^{(u)}(p, s), \\
& \frac{d \sigma}{d t}(a, b)_{y, r a-M S C}=\frac{d \sigma}{d \sigma}(a, b)_{\text {PRF. }}-\frac{d \sigma}{d \varepsilon}(a, b)_{\text {ere-MSD }},
\end{aligned}
$$

where $J_{a}$ ( $\left.E_{a}\right)$ is the cross section for forming the composite nucleus, when the projectile has energy $\varepsilon_{a}$. The evaporation or equilibrium components are determined by taking all of the input reaction cross section rot used in the preequilibrium phase of the reaction and aistributing it among the various reaction channels in proportion to their phase space. Only evaporation from the original compound nucleus is presori considered in the program. Subsequent emission from the residual nuclei is left out.

The energy differential evaporation cross section for the first emitted particle in a reaction is

$$
\frac{d \sigma}{d \varepsilon}(a, b)_{E V A P}=\left[\sigma_{a}\left(\varepsilon_{a}\right)-\sigma_{P R E}\right] \frac{w_{b}(\varepsilon)}{\sum_{b} \int w_{b}(\varepsilon) d \varepsilon},
$$

where the total preequilibrium cross section, $\sigma_{\text {?RE' }}$ is

$$
\sigma_{\mathrm{RF}}=\sum_{\mathrm{h}} \int \frac{\mathrm{d} \sigma}{\mathrm{dE}}(\mathrm{a}, \mathrm{b})_{\mathrm{PRE}} \mathrm{dE}
$$

\section{I.6 Pairing Corrections}

While preequilibrium pairing corrections are best made and studied in the two-component exciton model, they are 
sometimes included in the one-component model.

In the present code, two sets of pairing corrections are used: $\Delta_{\text {pre }}(Z, N)$ is used for states with $h \leq 2$, while the equilibrium pairing correction $\Delta_{\text {eq }}(2, N)$ is used for $h>2$. The quantity Apre may be zers, the equilibrium vallie or a special preequilibrium pairing correction. perhaps related to the number of broken proton or neutron pairs in states with $n=n_{0}[G R 73]\left[\mathrm{CN}^{-} \cdot\right.$. L Likewise, $\Delta_{\mathrm{cq}}(\mathrm{Z}, \mathrm{N})$ may also be iviken to re zero.

Whenever pairing energy corrections are ised, the quantity $E$ in the state density expressions should be replaced by $\left(E-\Delta_{p r e}\right)$ or $\left(E-\Delta_{e q}\right)$ depending on the value of $h$, and similarly for $U$ in the state densilies for the residual nuclei.

\section{DIRECT REACTION MODELS}

Two classes of direct reactions which are not included in the Griffin model are nucleon transfer (stripping, pickup and exchange) ana knockout or inelastic-scattering processes which involve complex particle degrees of freedom. These are treated semi-empirically in subroutines in PRECO-D. The formulae used are refined somewhat from those in [KA77]. 


\section{II.l Nucleon Transfer}

The basic equation for the eriergy differential cross section for nucleon transfer is

$$
\begin{aligned}
& \frac{d \sigma}{d \varepsilon}(a, b)_{N U}=\frac{\left(2 s_{b}+1\right)}{\left(2 s_{a}+1\right)} \frac{A_{b}}{A_{a}} \frac{E_{b-N E}(s)}{A_{a} \sigma_{a}} k_{\alpha, p}\left(\frac{A_{a}}{E_{a t}+V_{a}}\right)^{2 n}\left(\frac{2860}{A_{b}}\right)^{n}
\end{aligned}
$$

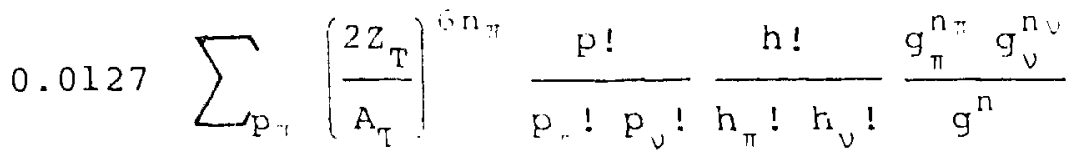

$$
\begin{aligned}
& w(p, h, j),
\end{aligned}
$$

where the subscripts a and $B$ refer to the projectile and residual nucleus. The inverse cross sections, $\sigma_{b}(\varepsilon)$, used in the main program are assumed to be the total composite nucleus formation cross section, while $\sigma_{b-N E}(\varepsilon)$ here is the total nonelastic cross section. The two are assumed to be related by

$$
\sigma_{b}(\varepsilon) \begin{cases}=0.95 \sigma_{b-N E}(E) & \text { for } b=\text { nucleon } \\ =0.85 \sigma_{b-N E}(E) & \text { for } b=\text { complex. }\end{cases}
$$

The quantities $E_{a}$ and $\bar{\varepsilon}_{a}$ are the laboratory and center of mass energies of the projectile, while $\mathrm{V}_{\mathrm{a}}$ is the average potential seen by the projectile in the direct reaction region. It is taken to be one fourth of the real central well depth or

$$
\mathrm{V}_{\mathrm{a}}=\frac{50 \mathrm{MeVA} \mathrm{A}_{\mathrm{a}}}{4}
$$

The constant $K_{\alpha, p}$ gives a factor of 12 enhancement to the 
nucleon transfer whenever the projectile and ejectile are both tightly tousi: (i..., a nucleon or an alpha particle). It is unity for all other reactions. The particle and hole numbers are given by $\mathrm{p}=\mathrm{p}_{\pi}+\mathrm{p}_{1,}$ and $\mathrm{h}=\mathrm{h}_{\pi}+\mathrm{h}_{1 i}$, where $\mathrm{p}_{\pi}, \mathrm{p}_{1,}, \mathrm{~h}_{\pi}$, ar.d $h$ are the numbers of stripped protons and neutrons and picked-up protons and neutrons, respectively. In general, these variables are uniquely determined by the natures of the incoming and outgoing particles. For inelastic channels of weakly bound projectiles ( $d, t$ or ${ }^{3} \mathrm{He}$ ), however, exchange of either a proton or a neutron with the target is allowed. Thus there will be two contributions to the nucleon transfer cross section, one with $\mathrm{P}_{\mathrm{T}}=\mathrm{h}_{-.}=1$ and $\mathrm{P}_{\mathrm{H}}=\mathrm{h}=0$, and the nther having $\mathrm{p}_{\pi}=\mathrm{h}_{\pi}=0$ and $\mathrm{p}_{i}=\mathrm{h}_{y}=\mathrm{l}$. This is the only case where the sum over $P_{\pi}$ is needed. Finally, $g_{\pi}$ and $g_{1}$ are the proton and neutron single-paricicle-state densitıes, assumed to be $q_{T}=g_{n}(Z / A)$ and $g_{0}=g_{0}(N / A)$ evaluated $i n$ the residual r.ucleus.

The nucleon-transfer cross section as given here will be in mb/MeV. All of the energies are assumed to be in MEV and the nonelastic cross sections in mb.

II. 2 Knockout and Inelastic Processes with Complex Particle Degrees of Freedom

In this type of mechanism, a comploy projectile is assumed to be able to excite a proton, neutron or alpha cluster particle/hole pair in its initial interaction and 
to retain its own cluster identity in the interaction. Nucleon pair excitation by a nucleon projectile is already considered in the exciton model, so only alpha cluster excitation is cossidered here for incident neutrons and protons. The basic equations for knockout and inelastic scattering are

$$
\begin{aligned}
& \frac{d \sigma}{d e}(a, b)_{K O}=\frac{\sigma_{a-N E}}{13.5}\left(2 s_{b}+1\right) A_{b} E \sigma_{b-N E}(E) \\
& \frac{P_{b} g_{a} g_{b}\left[U-A\left(A_{a}, A_{b}\right)\right]}{\sum_{c=a, b}\left(2 s_{c}+1\right) A_{c} \bar{\sigma}_{c}\left(E_{m}+2 B_{c}\right)\left(E_{m}-B_{c}\right)^{2}\left(g_{a} g_{b}^{2} / 6 g_{c}\right)}
\end{aligned}
$$

$\frac{d \sigma}{d \varepsilon}\left(a, a^{\prime}\right)_{I N E L}=\frac{\sigma a-N E}{13.5}\left(2 s_{a}+1\right) A_{a} E \sigma_{a-N E}(E)$

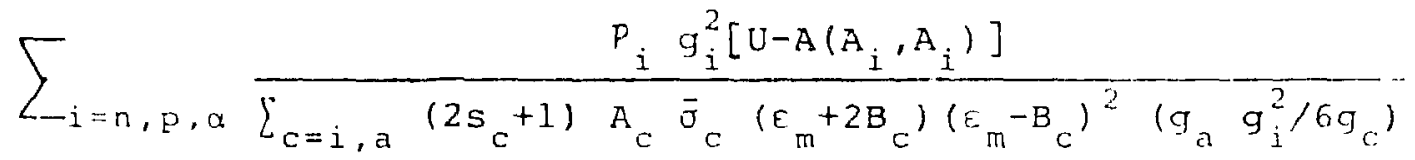$$
\text { for } a=\text { complex, }
$$

$\frac{d \sigma}{d \varepsilon}\left(a, a^{\prime}\right) I N E L_{I N}=\frac{\sigma-N E}{13.5}\left(2 s_{a}+1\right) A_{a} E \sigma_{a-N E}(\varepsilon)$

$$
\begin{aligned}
& \frac{P_{\alpha} g_{\alpha}[U-A(4,4)]}{\sum_{c=a, r}\left(2 s_{c}+1\right) A_{c} \bar{\sigma}_{c}\left(\varepsilon_{m}+2 B_{c}\right)} \frac{\left(\varepsilon_{m}-B_{c}\right)^{2}\left(g_{a} g_{(L}^{2} / 6 g_{c}\right)}{(U)} \\
& \text { for } \mathrm{a}=\text { nucleon }
\end{aligned}
$$

The quantity $\varepsilon_{m}$ is the energy of the ground statc transition for emission of a particle of type $c$, while $\sigma_{a-N E}$ with no energy variable specified is the nonelastic cross section for the entrance channel. The cross section $\bar{\sigma}_{c}$ is the average for all emission energies above the coulomb barrier 
for particles of type c. The probabilities for exciting the different kirids of particle/hole pairs are

$$
\begin{aligned}
& F_{n}=\frac{N_{T}-f Z_{T}}{A_{T}-2 f Z_{T}+f} \simeq \frac{N_{T} / 2}{A_{T}},
\end{aligned}
$$

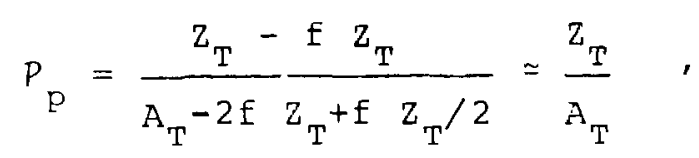

$$
\begin{aligned}
& P_{\alpha}=\frac{\mathrm{f} \mathrm{Z}_{\mathrm{T}^{\prime}}{ }^{\prime 2}}{\mathrm{~A}_{\mathrm{T}}-2 \mathrm{f} \mathrm{Z}_{\mathrm{T}}+\mathrm{f} \mathrm{Z}_{\mathrm{T}} / 2}=\frac{\mathrm{f} \mathrm{Z}_{\mathrm{T}}}{2 \mathrm{~A}_{\mathrm{T}}} .
\end{aligned}
$$

Here the quantity $f$ is defined as the fraction of the time that four nucleons in correlated orbits will "look like" an alpha ciuster or, aiternatively, the fraction of the possible alpha cluste:s that will, on the average, exist at any giver time. It has been assumed that $\mathrm{N} \geq \mathrm{Z}$, so that a maximum of $\mathrm{Z} / 2$ alpha clusters is possible. In general $f<r$. The exact size and systeratics of $f$ are not well known. Here the values obtains 1 from $(p, \alpha)$ reactions by neglecting pickup [NI74] are reiormalized slightly and parameterized to give
$\mathrm{f}\left\{\begin{array}{l}=0.08 \\ =0.02+0.06\left(126-\mathrm{N}_{\mathrm{T}}\right) / 10 \\ =0.02+0.06\left(\mathrm{~N}_{\mathrm{T}}-126\right) / 3 \\ =0.08\end{array}\right.$

$$
\begin{array}{r}
\mathrm{N}_{\mathrm{T}}<116 \\
116<\mathrm{N}_{\mathrm{T}}^{-}<126 \\
126<\mathrm{N}_{\mathrm{T}}<129 \\
1.29<\mathrm{N}_{\mathrm{T}} .
\end{array}
$$

In evaluating the single-particle-state densities for the different types of emitted particles, it is assumed that all of the nucleons of a cluster are in correlated orbits, each holding a maximum of two protons and two neutrons. In addition it should be recognized that a cluster of $A_{b}$ 
nucleons will carry $A_{b}$ times the energy of a single particle in one of these urbits. Thus we get

$$
\begin{aligned}
& g_{\mathrm{n}}=\quad g_{0} \mathrm{~N} / \mathrm{A}=(\mathrm{N} / 13) \mathrm{MeV}^{-1}, \\
& g_{\mathrm{p}}=g_{0} 2 / \mathrm{A}=(2 / 13) \mathrm{MeV}^{-1}, \\
& g_{\mathrm{d}}=g_{0} / 4=(\mathrm{A} / 52) \mathrm{MeV}^{-1}, \\
& g_{t}=g_{\tau}=g_{0} / 12=(\mathrm{A} / 156) \mathrm{MeV}{ }^{-1}, \\
& g_{a}=g_{0} / 16=(\mathrm{A} / 208) \mathrm{MeV}^{-1} .
\end{aligned}
$$

In each case they are evaluated in the appropriate residual nucleus. Once again, the final cross sections are in mb/MeV.

\section{ANGULAR DISTRIBUTIONS}

The angular distributions are calculated phenomenologically in terms of Legendre polynomials using the formula [KA8la]

$$
\begin{aligned}
\frac{d^{2} \sigma}{d \Omega d \varepsilon}(a, b) & =a_{0}(M S D) \sum_{\ell=0}^{6} b_{\ell}(e) p_{\ell}(\cos \theta) \\
& +a_{0}(M S C) \sum_{\substack{\ell=0 \\
\Delta \ell=2}}^{6} b_{\ell}(e) P_{\ell}(\cos \theta),
\end{aligned}
$$

where $e=\varepsilon+I_{a n g} \cdot B_{b}$ and $I_{a n g}$ is either 0 or 1 , and where

$$
a_{O}(M S D)=\frac{1}{4 \pi}\left(\frac{d \sigma}{d \varepsilon}(a, b)_{\text {pre-MSD }}+\frac{d \sigma}{d \varepsilon}(a, b)_{N U}+\frac{d \sigma}{d \varepsilon}(a, b)_{K O}\right) \text {. }
$$

(For the reaction $\left(a, a^{\prime}\right)$ the knockcut contribution is, of course, replaced by an inelastic contribution.)

$$
a_{0}(M S C)=\frac{1}{4 \pi}\left(\frac{d \sigma}{d \varepsilon}(a, b)_{\text {pre-MSC }}+\frac{d \sigma}{d \varepsilon}(a, b)_{\text {EVAP }}\right),
$$




$$
\begin{aligned}
& b_{\ell}(E) \begin{cases}=\frac{(2 \ell+1)}{1+\exp \left[A_{k}\left(B_{k}-e\right)\right]} & \text { for } \ell \geq 1 \\
=1 & \text { for } \ell=1,\end{cases} \\
& A_{\ell}=0.036 \mathrm{MeV}^{-1}+0.0039 \mathrm{MeV}^{-1} \ell(\ell+1) \text {, } \\
& \mathrm{B}_{\ell}=\left[92+6\left(I_{\mathrm{ang}}\right)\right] \mathrm{MeV}-90 \mathrm{MeV}[\ell(l+1)]^{-1 / 2} \text {. }
\end{aligned}
$$

\section{ISOSPIN}

For reactions induced by protons or ${ }^{3}$ He ions with suftıcient energy, states in the composite nucleus with two different total isospins can be populated: $\mathrm{T}_{<}=\mathrm{T}_{\mathrm{z}}$ and $\mathrm{T}_{>}=\mathrm{T}_{\mathrm{z}}+1$. Therefore, it may sometimes be desirable to perform preequilibrium calculations in which the isospin quantum number is conserved [KA74].

To perform such isospin-conserved calculations, it is necessary to know the state densities for both the $\mathrm{T}_{<}$and T, configurations in a given nucleus. At equilibrium it is usually a reasonable approximation to take

$$
\begin{aligned}
& \omega\left(E, T_{<}\right)=w(E), \\
& \omega\left(E, T_{>}\right)=\omega\left(E-E_{\text {sym }}(Z, A)\right),
\end{aligned}
$$

where $E_{\text {sym }}(Z, A)$ is the nuclear symmetry energy and can be obtained from the empirical formula [fN65]

$$
E_{\text {sym }}(Z, A)=1.44 \mathrm{MeV} \frac{Z+1 / 2}{A^{1 / 3}}-1.13 \mathrm{MeV}+Q_{(p, n)}(Z-1, A),
$$

where $Q_{(p, n)}$ is the $Q$-value for the $(p, n)$ reaction on the nucleus with the indicated proton and mass numbers. 
For particle/hole state densities, a similar

approximation has been used [KA74] giving

$$
\begin{aligned}
& w\left(P, h, E, T_{<}\right)=w(p, h, E), \\
& w\left(p, h, E, T_{>}\right)=w\left(p, h, E-E_{s y m}(Z, A)\right.
\end{aligned}
$$

Sinilar ussumptions are assumed to apply for the T-conserving transition-state densities.

While such reiations are the only ones which can currently be used in PRECO-D because isospin is not explicitly considered, they have a serious shortcoming for small values of $n=p+h$ where the state densities increase only as $E^{n-1}$ [KA84]. Thus, isospin-dependent calculations are probably better carried out using the two-component exc,ton model code PRECO-E [KA84a] in which more accurate state density formulae are used.

In either code two calculations are performed, one for each isospin in the composite nucleus. The reaction cross sections are multiplied by the appropriate isospin clebschGordan coefficients and the state densities are evaluated using excitation energies measured relative to the lowest energy state of the isospin in question.

\section{DESCRIPTION OF THE PROGRAM}

The program PrECO-D2 is written in simple FORTRAN designed to be compatible with most computer systems. A general flow diagram is contained in Fig. 1; a list of variables is given 
In Tables 1 and 2; Appendix $A$ is a FORTRAN 1 isting of the code; Appendix B contains sample input; Appendix C gives the corresponding output; and Appendix D lists the differences between PRECO-D2 and its predecessor, PRECU-D.

Input and output device numbers are assigned as IREA and IWRI, respectively, just following the COMMON statements, so they may be easily altered.

The Griffin, or exciton, model calculations are carried out in the main program, while direct reaction cross sections and angular distributions are calculated in subroutines. The subroutines NUTRA, KNOCK, and INEL handle, respectively, nucleon transfer, knockout involving cluster degrees of freedom, and inelastuc scattering involving cluster degrees oi freedom. The subroutine PoLLy calculates a library of Legendre polynomials, while ANGEL generates the angular distributions for the emitted particles.

Reaction cross sections for the projectile and inversereaction cross sections for the emitted particles may, as an option, be calculated internally using an empirical approximation $[\mathrm{NA79}][\mathrm{CH} 81]$. It ras been modified here to zo over to the geometrical cross section

$$
\sigma_{b-G E O}(E)=\pi\left(1.23 \mathrm{~A}^{1 / 3}+\mathrm{R}_{\mathrm{b}}+\pi\right)^{2}
$$

at high energies, where $R_{b}$ is the radius of the particle $(0$ for $b=n, p ; 0.8 \mathrm{~F}$ for $b=d, t, \tau ;$ and $1.2 \mathrm{~F}$ for $b=\alpha$ ). The parameter values are set in subroutine SIGPAR, while the entrance and exit channel cross sections are calculated in subroutine cRoss and the moin program, respectively. 
Initial calculations are always performed for the principal reaction channels of neutron, proton and alpha particle emission. Fission competition j.s included at equilibrium if a fission barrier has been specified. Spectra for other ejeated light ions may be calculated at a later stage in the proqram. In the setup and calling of the direct reaction subroutines, it is assumed that both the incoming and outgoing particles have mass numbers no larger than 4 . The range of exciton numbers considered is determined from the input initial configuration, $\left(\mathrm{p}_{0}, \mathrm{~h}_{0}\right)$, and the state density, $w(P, h, E)$. The difference $p-h$ is assumed to remain constant at $\mathrm{P}_{0}{ }^{-h_{0}}$. The smallest $\mathrm{n}=\mathrm{p}+\mathrm{h}$ considered is the minimum value consistent with there being at least one degree of freedom to carry the excitation energy and with both $\mathrm{p}$ and $\mathrm{h}$ being nonnegative. In must cases $\left(\mathrm{r}_{\mathrm{O}}, \mathrm{h}\right)=$ $\left(A_{a}+1,1\right)$, so the simplest configuration of the system is $\left(A_{a}, 0\right)$. At the other extreme, the most complex configuration considered has its exciton number given by minimum $(\bar{n}, 20)$, where $\bar{n}$ is the exciton number corresponding to the maximum in the $w(p, h, E)$ vs $p$ curve and is therefore the most probable equilibrium value of $n$.

For partisle emission to be allowed from a state, its particle number most ba greater than or equal to the nucleon number of the emitted particle $\left(i . e ., p \geq A_{b}\right)$ and $n$ must be large enough for the residual nucleus to be left with at least one exciton $\left(i . e, n \geq A_{b}+1\right)$. 
VI. USE OF THE PROGRAM

VI.l Initial Input

The input information requested for the first phase of the calculations is given below. Each numbered item corresponds to a new card or record of input, and the appropriate formats are given in parentheses. Default values are also indicated where they exist.

L) Angular distribution parameter, Iang, and Q-factor parameter, IG, (2I3) for all problems in the data file.

i.) Effective well depths $V(h=1)$ and $V(h=2)$ in $M e V(2 F l 0.2)$ for all problems in the data file.

1) Composite nucleus excitation energy and projectile binding energy in $\mathrm{MeV}$ (2F10.2).

2) $\mathrm{Z}$ and $\mathrm{N}$ of the target nucleus (2Fl0.2)

3) $n, p$, and $\alpha$ binding energies and fission barrier height in the composite nucleus, all in $\mathrm{MeV}(4 \mathrm{Fl0.2})$. If no fission barrier is specified, no fission is calculated.

4) Pairing energies for the $n, p$, and $\alpha$ residual nuclei and for the composite nucleus to be used in the preequilibrium phase of the calculation for states with $\mathrm{h} \leq 2 \quad(4 \mathrm{~F} 10.2)$.

5) Pairing energy corrections for states with $h>2$ and for equilibrium calculations. These are the conventional fairing energies that would be used in an evaporation model. (4Fl0.2).

6) Entrance channel nonelastic cross section and estimated average, $\sigma_{a}$, for inelastic scattering (2Fl0.2). If either one is read in as zero, it is calculated internally using a parametric approximation to the optical model potentials indicated in Sect. VI.7 below.

7) Number of channel energies considered, NEPS(1), the lowest channel energy in $\mathrm{MeV}$, and the increment in the channel energy also in $\mathrm{MeV}(1 \mathrm{I3}, 2 \mathrm{Fl0.2)}$. If the lowest channel 
energy is greater than zero, the remaining channel energies and all of the inverse reaction cross sections are calculated internaliy. (IE the channel energy increment is zero, it defaults to $1 \mathrm{MeV}$.) If the lowest channel energy is read in as zero, input according to item $7 a$, below, is read in.

7a) NEPS(1) records, each containing (4Fl0.2) a channel energi in Mer and the corresponding $n, p$ and ix nonelastic cross sections in $\mathrm{mb}$. The energies must be in ascending order.

8) $Z$ and $N$ of the projectile (2I3). If preferential excitation of an unpaired target nucleon is considered, then this nucleon should be counted as if it were part of the projectile.

9) Initial particle and hole numbers, $p$ and $h$ (2I3). If these are read in as zero, po defäults tó $A_{a}+1$ and ho defaults to unity.

10) Singla-particle-state densities, go, in $\mathrm{MeV}^{-1}$ for the composite system; the $n, p$ and $\alpha$ residual nuclei; and the fission saddle point (5Fl0.2). If only the value for the composite nucleus is read in, the others are calculated assuming that $g_{0}$ is proportional to $\mathrm{A}$. If $g_{0}$ for the composite nucleus is read in as zero, it defaults to $\mathrm{A} /(13 \mathrm{MeV})$.

11) Matrix element scalind factor divided by 100 ( $i \cdot c^{2}$, $\mathrm{k} / 100)(\mathrm{Ll0.2)}$. If it is read in as zero, it defaults to $1.35 \quad(k=135 \mathrm{MeV})$.

VI. 2 Initial Calculations

After initial input, the program proceeds to the calculation of state densities, transition rates and particle emission rates. The branching ratios, $\Gamma$, are generated and the closed-form calculations are executed.

At this point, the three principal particle types $(n$, $p$, and $\alpha$ ) are considered in turn. For each, the recessary direct reaction subroutines are called to generate the 
appropriate energy spectra. The results of the closed-form exciton model calculations are used to obtain MSD and MSC preequilibrium energy spectra. A first-chance evaporation spectrum is also calculated. For each emission energy the total MSD and MSC cross sections are used in the subroutine ANGEL to calculate ail angular distribution. Finally, the single and double differential cross sections are printed out.

VI.3 Initial Output

The first quantities to be printed are $s$, the average effective separation energy, and the fraction of the total reaction cross section which goes into preequilibrium particle emission. These are part of a heading labelled OCCUPATION PROBABILITIES. Below the heading is a table with the following columns:

P particle number of the class of states

$\mathrm{H}$ hole number of the class of states

RHOU/RHO ratio $\omega^{(u)}(p, h, E) / \omega(p, h, E)$ of unbound and total state densities

STRU/STR ratio $P_{u}(p) / P(p)$ of strength arriving at states with $p$ particle degrees of freedom which is in unbound states

$S T R D / S T R$ ratio $P_{A}(p) / P(p)$ of MSD strength to total strength arriving at states specified by $p$

Following this are two pages of output for each particle type, one containing the energy differential spectra and the other the angular distributions for the various outgoing energies. The column headings for the angle-integrated 
spectra are

EPS

DIRECT/NUTRA

DIRECT /KNOCK e, the outgoing channel energy

nucleon transfer cross section from Eq. (48)

knockout or inclastic cross section Erom Eq. (51) or (52)

MSD preequilibrium cross section from $\mathrm{Eq} \cdot(45 \mathrm{~b})$

MSC preequilibrium cross section from Eic. $(45 \mathrm{c})$

evaporation cross section from Eq. (46)

"NUTRA+ "KNOCK" + "PREEQUILIBRIUM/MSD"

"PREEQUILIBRIUM/MSC" + "EQUIL/WEISS "

TOTAL/MSC

TOTAL/MSD+MSC grand total

At the bottom of each column is the summed cross section for that componert of the spectrum. If a fission barrier has been specified, the equilibrium fission cross section is printed below the sum of EQUIL/WEISS for the alpha spectra.

The angular distributions are printed in 10-deg increments. Angles from 0 to 90 deg are in one block of the tablc, while angles from 100 to $180 \mathrm{deg}$ are in a second block. The first column of each block gives the channel energy; the remaining columns give the double differential cross section at the angle indicated in the column heading. The last column on the second block gives the angle-integrated cross sections and is identical to TOTAL/MSD+MSC above. 
VI. 4 Secondary Input

After printout of the occupation probabilities and of the neutron, proton and alpha particle spectra, the program reads the secondary input, which contains the following items :

12) $Z$ and $N$ of an additional particle type (2I3). (If $z<0$, input passes to item l3a below.)

13) Spin degeneracy, 2s+1, of the emitted particle (II3)

14) binding energy of emitted particle in MeV and the single-particle-state density in its residual nucleus in $\mathrm{MeV}^{-1}(2 \mathrm{Fl0.2})$. If the single-particle-state density is zero, it defaults to $\left(g_{0} A_{\text {res }} / A_{C n}\right)$.

15) preequilibrium $(h<2)$ and equilibrium pairing corrections for the residual nucleus in $\operatorname{MeV}$ (2F10.2)

16) number of emitted particle energies considered = NEPS(2), the lowest emission channel energy, and the channel energy increment (1I3,2Fl0.2). If the lowest energy is greater than zero, the remaining energies and the inverse cross sections are calculated internally. (A zero chanuel energy increment defaults to $1 \mathrm{MeV}$. ) If the minimum e.sergy is zero (or less), input item $16 \mathrm{a}$ is read in.

$16 a$ NEPS(2) records, each containing a particle energy in $\mathrm{MeV}$ and the corresponding nonelastic ross section in $\mathrm{mb}(2 \mathrm{Fl0.2})$. Energies must be in increasing order. [This ends the secondary input.]

13a) NEWP (1I3)

IE NEWP $=1$, irput passes to item 8 in the initial input and new parameter values are read in.

If iNEW - 0, input passes to item 1 in the initial input for the beginning of a new problem.

If NEWP $=-1$, the job is terminated. 


\section{VI.5 Secondary Calculations}

If a new particle type was shosen in item 12) above, the program us.:s the stored information on the results of the ciosud-frrm calculations to obtain the pruequilibrium spectra for this particle type, and the appropriate direct reaction subroutines are called. The single and double differential cross sections are then printed in tho same format as for the noutron, proton and alpha particle spectra. After printout, the program returns to item 12 ) in the secondary input so that an additional particle type may be specified.

VI. 6 Calculations with Isospin

Preequilibrium model calculations in which isospin conservation is considered can be accomplished with PRECO-D2 by actually doing two calculations, one for each isospin in the composite nucleus. The changes in the input needed for the isospin-conserved calculations relative to the general (isospin-mixed) calculations just described are indicated in the table below for the initial input. All other quantities in the initial input are as indicated in VI.I and VI. 4 above. In the table, the second set of input for $B_{n}$ and $\sigma_{n-N E}(E)$ for the $T$, calculations applies if the neutron channel to the $T$, states in the residual nucleus is open. 
Neutrons decay from the $T$, composite nucleus states to the ground state isospin if the residual nucleus is isospinforbidden.

\begin{tabular}{|c|c|c|}
\hline Normal & $\mathrm{T}_{<}$ & $\mathrm{T}>$ \\
\hline$E$ & $E$ & $E-E_{s y m}(Z, A)$ \\
\hline $\mathrm{B}_{\mathrm{n}}$ & $\mathrm{B}_{\mathrm{n}}$ & $\left\{\begin{array}{l}0 \\
B_{n}-E_{s y m}(Z, A)+E_{s y m}(Z-1, A)\end{array}\right.$ \\
\hline $\mathrm{B}_{\mathrm{p}}$ & $\mathrm{B}_{\mathrm{p}}$ & $B-E_{s y m}(Z, A)$ \\
\hline $\mathrm{B}_{\mathrm{a}}$ & $\mathrm{B}_{a}$ & 0 \\
\hline$\sigma_{2}-N E$ & $\sigma_{\mathrm{a}-\mathrm{NE}}\left[2 \mathrm{~T}_{\mathrm{O}} /\left(2 \mathrm{~T}_{\mathrm{O}}+1\right)\right]$ & $\sigma_{\left.\left.a-N E^{[1 /(2 T}+1\right)\right]}$ \\
\hline$\sigma_{n-N E}(E)$ & $\sigma_{n-N E}(E)$ & $\left\{\begin{array}{l}0 \\
\sigma_{n-N E}(E)\left[2 T_{0} /\left(2 T_{0}+1\right)\right]\end{array}\right.$ \\
\hline$\sigma_{p-N E}(E)$ & $\sigma_{I-N E}(\varepsilon)\left[2 \mathrm{~T}_{0} /\left(2 \mathrm{~T}_{0}+1\right)\right]$ & $\sigma_{p-N E}(E)\left[1 /\left(2 T_{0}+1\right)\right]$ \\
\hline$\sigma_{\alpha-N E}(E)$ & $\sigma_{\alpha-N E}(E)$ & 0 \\
\hline k & $k$ & $k\left[E-E_{\text {sym }}(Z, A)\right] / E$ \\
\hline
\end{tabular}

VI.7 Suggested Input

Appropriate values for the various input quantities are summarized below
$\left(p_{0}, h_{0}\right)$
$\left(A_{a}+1,1\right)$, where $A_{a}$ is the mass number of the projectile (the default values).
IJ
A/13 with values for the residual nuclei calculated in the program (the default).
k
$135 \mathrm{MeV}^{3}$ (input number is 1.35) (the default).
$\mathrm{B}_{\mathrm{b}}$
from [WA77] or similar mass tables 


$$
\begin{aligned}
& J_{\therefore-\because E}(E) \text { from }[\text { MA63] } \\
& \because-\therefore E(c) \quad \text { Erom }[B E 69] \\
& C_{a-N E}(E) \quad \text { from [HUG2] } \\
& a_{b-N E}(E) \quad \text { from }[\mathrm{CL} 72] \text { for } b=a, t,{ }^{3} \mathrm{He} \\
& \text { (The default is } \\
& \text { approximations to } \\
& \text { these optical model } \\
& \text { cross sections.) }
\end{aligned}
$$




\section{REFERENCES}

[AN65] J. D. Anderson, C. Wong and J. W. McClure, Phys. Rev. 138 (1965) B615

[BE69] F. D. Becchetti, Jr. and G. W. Greenlees, Phys. Rev. 182 (1969) 1190

[CH81] A. Chatterjee, K. H. N. Murthy and S. K. Gupta, Pramāna 16 (1981) 391

[CL72] C. K. Cline, Nucl. Phys. Al93 (1972) 417

[GA77] E. Gadioli, E. Gadioli-Erba and J. Hogan, Phys. Rev. C. 16 (1977) 1404

[GR66] J. J. Griffin, Phys. Rev. Le+t. 17 (1966) 478

[GR73] S. M. Grimes, J. D. Anderson, J. C. Davis and C. Wong, Phys. Rev. C 8 (1973) 1770

[GR76] S. M. Grimes, J. D. Anderson, and C. Wong, Phys. Rev. C 13 (1976) 2224

[HU62] J. R. Huizenga and G. Igo, Nucl. Phys. 29 (1962) 462

[KA74] C. Kalbach-Cline, J. R. Huizenga and H. K. Vonach, Nucl. Phys. A222 (1974) 405

[KA77] C. Kalbach, Z. Phys. A283 (1977) 401

[KA78] C. Kalbach, Z. Phys. A287 (1978) 319

[KA31] C. Kalbach, Phys. Rev. C 23 (1981) 124; and Phys. Rev. C 24 (1981) 819

[KA8la] C. Kalbach anả F. M. Mann, Phys. Rev. C 23 (1981) 112; and C. Kalbach, Phys. Rev. C 25 (1982) 3197 
[KA83] C. Kalbach, in BNL-NCS-51694 (proceedings of the IAEA advisory working group meeting on basic and applied problems of nuclear level densities, edited by M. R. Bhat) June 1983, p 113

[KA84] C. Kalbach, "Isospin Dependence of Two-Component Particle-Hols State Densities for Nuclei," Phys. Rev. C 30, p $1310(1984)$.

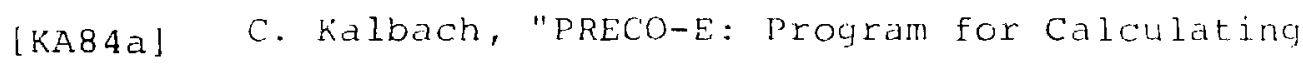
Freequilitrium and Direct Reaction Double DifFerentad Cross Sections usirg the T'wo-Component. Exciton Model" (to be published as a Los Alumos informal document).

[MA63] G. S. Mani, M. A. Melkanoff and I. Iori, Centre d'Etudes Nucleaires de Saclay report CEA 2380 (1963)

[MI74] L. Milazzo-Colli et al., Nucl. Phys. A218 (1974) 274; Nuov. Cim. 30A (1975) 632; Nuov. Cim. 39A (1977) 171

[NA79] K. H. Narasimha Murthy, A. Chatterjee and S. K. Gupta, Proc. Int'l Conf. on Nucl. Cross Sections for Technology, Knoxville, 1979, NBS Spec. Pub. 594, p 793

[WA77] A. H. Wapstra and K. Bos, Nucl. Data Tables 19 (1977) $2 i 5$ 


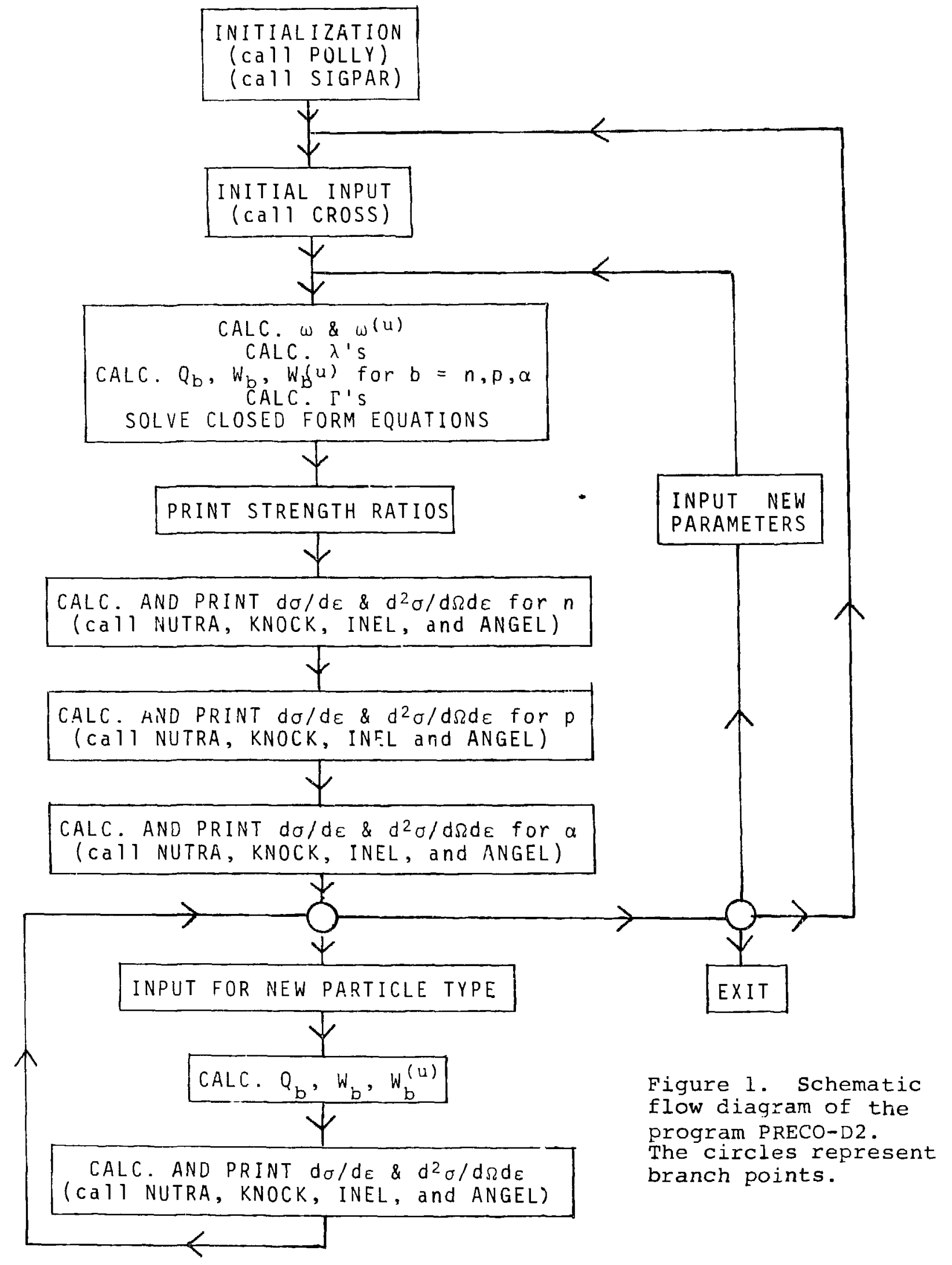


ACOM

AP

ASP , ASPR, AFISH

BEA

$B E N$

$\mathrm{BF}$

BNEU , BPRO, BALFA

CLOSD (NP)

CLOSU (NP)

COUL (J)

DBL (NP)

E

EC

$\operatorname{EMU}(\mathrm{J})$

EPS (JHALF, NE)

ES (I)

F2
Mass number of composite nucleus

= JouT, mass number of emittrd particle

Level spacing parameter, a, for composite and residual nuclei and for top of fission barrier

Binding energy of projectile in composite nucleus (MeV)

Binding energy for extra emitted particle types (or temporarily of $n, p$ or $\alpha$ ) (MeV)

Height of fission barrier (MeV)

Binding energies of neutrons, protons and alpha partiales in the composite nuclev.

Time-integrated strength, $S_{d}(p) T_{u}(p)$, responsible for MSD preequiiibrium cross section

Time-integrated strength, $S_{U}(p) T_{u}(p)$, responsible for MSD+MSC preequilibrium cross section

Coulomb barriers for particles of type $\mathrm{J}$, with $J=1-5$ corresponding to $n, p, \alpha$, extra particle type and projectile, respectively; used in calculating direct reaction cross sections

$m(p)$, fraction of multiple unbound states

Excitation energy of composite nucieus (MeV)

Coulomb barrier for use in calculating totalreaction cross sections ( $\mathrm{MeV}$ )

Maximum possible emission energy for particles of type $\mathrm{J}$ with $\mathrm{J}=\mathrm{l}-3$ for $n, p$ and $\alpha$

Particle emission energies (MeV)

$\theta(E-i S)(E-i S) /(E-S)$ for $i=1,10$

Matrix element scale factor, $k$ 
FISHW

FLOW, SPILI, POUR

FRAC (NP)

FWD (NH)

FWDHI (NP)

FWDU (NP)

$G$, GNEU, GPRO, GALFA, GR, GFIS

$\mathrm{GF}, \mathrm{GFU}, \mathrm{GU}, \mathrm{GFAV}$

GFPU , GFHU

GAMBB, SAMBU

GAMUB, GAMUU

GAMZBU, GAMZUB

GEL (NP)

GELU (NP)

GELUU (NP)

IANG

IG

IREA, IWRI

JBAR

JHALF

JHI
Equilibrium cross saction going into fission $10^{-18}, 10^{+18}$, and $10^{+35}$; comparison numbers for underflow and overflow checks

Fraction of the available strength in states with $p=N P$ which is lost due to $n, p$ and $\alpha$ emission in one unit of time, $\Delta t=T A U W M$

Correction factor, $f(p)$, applied to $w(p, h, E)$ for $h<2$ to account for the finite potential well depth

$f_{+}(p)$ and (for $h>2$ ) $f_{0}(p)$

$\mathrm{f}_{\mathrm{I}}(\mathrm{p})$

Single-particle-state densities in the composite nucleus; in the $n, p, \alpha$ and extra particle residual nuslei; and at the top of the fission barrier

$g(p), g_{u}(p), g_{p}(p)$ and $g_{a}(p)$ for $h \leq 2$

$g_{p}(p)$ for $h>2$ and $g_{h}$ for all $h$

$\Gamma_{+}^{(b b)}(p), \Gamma_{+}^{(b u)}(p), \Gamma_{+}^{(u b)}(p)$; and $\Gamma_{+}^{(u u)}(p)$

$\Gamma_{0}^{(b u)}(p)$ and $\Gamma_{0}^{(u b)}(p)$

$\mathrm{g}[\mathrm{E}-\mathrm{A}(\mathrm{P}, \mathrm{h})]$

$g\left[E-A_{1,0}(p, h)-S\right]$ or 0 , whichever is greater $g\left[E-A_{2,0}\left(p, h_{1}\right)-2 S\right]$ or 0 , whichever is greater Angular distribution parameter. Energy parameter is $E+\left(\right.$ IANG) $\left(B_{b}\right)$

$I G=0$ invokes $Q_{b}(p) ; I G=1$ invokes $Q_{b}^{(G)}(p)$

Input and output device numbers

Most probable particle number at equilibrium

JHALF=1 for primary calculations; JHALF=2 for secondary calculations (for additional

emitted particle types)

minimum of 20 and JBAR; maximum $p$ considered in ciosed-form reaction equations 
JLO

JPIN, JNIN, JIN

JRZ, JRN

JPOUT, JNOUT, JOUT

$N E$

NEPS (1), NEPS (2)

NEWP

NLOE

NAP 1

NP

NPART', NHOLE

NPHD, PHD

NDWD, NHDWN

NUP

NSD , SD

PRET2

$P U, P B, P M S D$

PRMSC, PRMSD

$\operatorname{PROB}(J, N P)$

PTOT

$\mathrm{P} 0, \mathrm{P} 1, \mathrm{P} 2, \mathrm{P}$
Minimum $p$ which can supply the protons and neutrons for the emitted particle

$\mathrm{Z}, \mathrm{N}$ and $\mathrm{A}$ of the projectile

$\mathrm{Z}$ and $\mathrm{N}$ of target

$Z, N$, and $A$ of emitted particle

Index for emission channel energies

Number of emitted particle energies for primary and secondary calculations

Control variable for selecting new values of the model parameters or recycling to a new problem

Particle number of simplest states which can undergo nucleon emission

Particle number of simplest states which can undergo emission of a given type of complex particle

Index for particle number, $p$, of states

Initial particle and hole numbers, $p_{0}$ and $h_{0}$ $\mathrm{p}-\mathrm{h}=\mathrm{p}_{\mathrm{o}}-\mathrm{h}_{\mathrm{o}}$

Lower limit on particle and hole numbers Maximum particle number accessible to system Spin degeneracy of emitted particle Full preequilibrium cross section for a given reaction and emission energy

$\mathrm{P}_{u}(\mathrm{p}), \mathrm{P}_{\mathrm{b}}(\mathrm{p})$ and $\mathrm{P}_{\mathrm{d}}(\mathrm{p})$; strengths arriving at states of given $p$ by pair creation for the first time

MSC and MSD preequilibrium cross sections $Q_{b}(p)$ or $Q_{b}^{(G)}(p) ; J=1,2,3,4$ indicates $b=n, p, c$ and extra pariicle type, respectively

Fraction of strength left at end of preequilibrium calculations

Parameters $p_{0}, p_{1}, p_{2}$, and $p$ for calculating entrance channel and inverse cross sections 


\begin{tabular}{|c|c|}
\hline $\mathrm{RHO}$ (NP) & $\omega(p, h, E)$ \\
\hline RHOU (NP) & $\omega^{(u)}(p, h, E)$ \\
\hline $\mathrm{RA}$ & $\begin{array}{l}\text { adius of incident or emitted particle (used } \\
\text { in calculating total-reaction cross sections) }\end{array}$ \\
\hline RNN , RZZ & $\mathrm{N}$ and $\mathrm{z}$ of target (same as JRN and JRZ) \\
\hline $\mathrm{RN}, \mathrm{RZ}$ & $\mathrm{N} / \mathrm{A}$ and $\mathrm{Z} / \mathrm{A}$ of target \\
\hline$S$ & Average effective separation energy \\
\hline SIGCN & Entrance channel nonelastic cross section \\
\hline $\operatorname{SIGIN}(J, N E)$ & $\begin{array}{l}\text { Inverse nonelastic cross sections; } J=1-4 \\
\text { has the same significance as for PROB }\end{array}$ \\
\hline $\operatorname{SIGBAR}(J)$ & $\begin{array}{l}\text { Average nonelastic cross section for } \\
\text { channel J; J=1-5 has same significance as } \\
\text { for coul }\end{array}$ \\
\hline SIZE & $g_{\circ} E$ \\
\hline SNOCK & $\begin{array}{l}\text { Cross section for knockout or inelastic } \\
\text { scattering involving cluster degrees of } \\
\text { Ereedom }\end{array}$ \\
\hline SNUTRA & Cross section for direct nucleon transfer \\
\hline STRB & Strength in bound states, $=S_{b}$ \\
\hline STRC & strength in unbound states, $=S_{u}$ \\
\hline SUMKE, SUMNE & $\begin{array}{l}\text { Energy integrals of the cross sections } \\
\text { SNOCK and SNUTRA }\end{array}$ \\
\hline SUMSC, SUMSD & $\begin{array}{l}\text { Energy integrals of the cross sections } \\
\text { PRMSC and PRMSD }\end{array}$ \\
\hline SUMW & Energy integral of the cross section WEISS \\
\hline TAUWM & $\begin{array}{l}\text { Time unit, } \Delta t, \text { for evaluating transition } \\
\text { rates, }=\left[20 \lambda_{+}\left(p_{m i n}, h_{m i n}\right)\right]^{-1}\end{array}$ \\
\hline TAUWU (NP) & $\begin{array}{l}\text { Lifetime of an unbound state against all } \\
\text { two-body interactions considered and } n, p \\
\text { and } a \text { emission; }=T_{u}\end{array}$ \\
\hline $\begin{array}{l}\text { THIBB, THIBU, } \\
\text { THIUB, THIUU }\end{array}$ & $\begin{array}{l}\text { Pair-creation rates } \lambda_{+}^{(b b)}, \lambda_{+}^{(b u)}, \lambda_{+}^{(u b)}, \\
\text { and } \lambda_{+}(u u)\end{array}$ \\
\hline
\end{tabular}




\begin{tabular}{|c|c|}
\hline TLOBU, TLOUB & Pair-annihilation rates $\lambda \stackrel{(b u)}{-}$ and $\lambda(u b)$ \\
\hline TNOBU, TNOUB & Exciton-scattering rates $\lambda_{0}^{(b u)}$ and $\lambda_{0}^{(u b)}$ \\
\hline TIMHI & $\lambda_{+}\left(P_{m i n}, h_{m i n}\right)$ \\
\hline $\begin{array}{l}\text { TOMSC (NE) } \\
\text { TOMSD (NE) }\end{array}$ & $\begin{array}{l}\text { Total MSC and MSD cross sections for a } \\
\text { given emission energy }\end{array}$ \\
\hline $\mathrm{U}$ & Residual nucleus excitation energy \\
\hline $\mathrm{WU}(\mathrm{J}, \mathrm{NP}, \mathrm{NE})$ & $\begin{array}{l}W_{b}^{(u)}(p, h, E) \text { with } J=1-4 \text { having the same } \\
\text { significance as for PROB }\end{array}$ \\
\hline WEISS $(J, N E)$ & $\begin{array}{l}\text { Weisskopf-Ewing evaporation cross section; } \\
J=1,4 \text { as for PROB }\end{array}$ \\
\hline XLO, XLI & $\begin{array}{l}\text { Parameters } \lambda_{0} \text { and } \lambda \text { for calculating entrance- } \\
\text { channel and inverse-reaction cross sections }\end{array}$ \\
\hline XLAMB & $\begin{array}{l}\text { Parameter } \lambda \text { for calculating entrance-channel } \\
\text { and inverse-reaction cross sections }\end{array}$ \\
\hline$X M O, X M I, X M U$ & $\begin{array}{l}\text { Parameters } \mu_{0}, \mu_{1} \text { and } \mu \text { for calculating } \\
\text { entrance-channel and inverse-reaction cross } \\
\text { sections }\end{array}$ \\
\hline $\mathrm{XNO}, \mathrm{XN} 1, \mathrm{XN} 2, \mathrm{XNU}$ & $\begin{array}{l}\text { Parameters } v_{0}, v_{1}, v_{2} \text {, and } v \text { for calculating } \\
\text { entrance-channel and inverse-reaction cross } \\
\text { sections }\end{array}$ \\
\hline
\end{tabular}


TABLE 2. ADDITIONAL VARIABLES IN SUBROUTINES

ANGEL

$\begin{array}{ll}\text { AL,BL } & \begin{array}{l}\mathrm{A}_{\ell} \text { and } \mathrm{B}_{\ell} \text { for calculating Legendre polynomial } \\ \text { coefficients }\end{array} \\ \text { TOMSC, AOMAD } & \begin{array}{l}\text { TOMSC and TOMSD from MAIN, each divided by } \\ 4 \pi ;=a_{0} \text { (MSC) and } a_{0} \text { (MSD) }\end{array} \\ \text { EPSCM } & \text { EPS (JHALF, NE) } \\ \text { POL }(J, L+1) & \begin{array}{l}\text { Legendre polynomial of order } L \text { for an angle } \\ \text { Of } 10(J-i) \text { degrees; (generated in subroutine } \\ \text { POLLY) }\end{array} \\ \text { SIGMA } & \text { Double differential cross section }\end{array}$

CROSS

(none)

I NEL

VMA , LYAX

GEN, GBA

Maximum emission energy and maximum residual nucleus excitation energy

Nucleons and alpha particles in the residual nuclei formed by the knock-out of these particles

GI , GIR

Cluster single-particle-state density in the residual nuclei formed from emission of the cluster and from reemission of the projectile

KNOC

EMAX, UMAX

(as in TNEL;

$G A, G B R, G B T$

Projectile singie-particle-state density in the residual nucleus and emitted-particlestate densities in the residual and target nuclei 
NUTRA

GN, GP

JPICKN, JPICKP

JSTRIN, JSTRIP

TRANN, TRANP

UMAX

VAB

VELO

POLLY

ANG

$\operatorname{POL}(J, L+1)$ $y_{v}$ and $y_{\pi}$ in the residual nucleus

Number of picked-up neutrons and protons

Number of stripped neutrons and protons

Number of transferred neutrons and protons

(as in INEL)

Effective potential, $V_{a}$, seen by the projectile in the interaction region

Projectile velocity in the interaction region

Angle, $\theta$, first in degrees and then in radians

(as in ANGEL)

SIGPAR

(none) 


\section{APPENDIX A}

\section{FORTRAN LISTING OF PRECO-D2}

Los Alamos Identification No. LP-1654

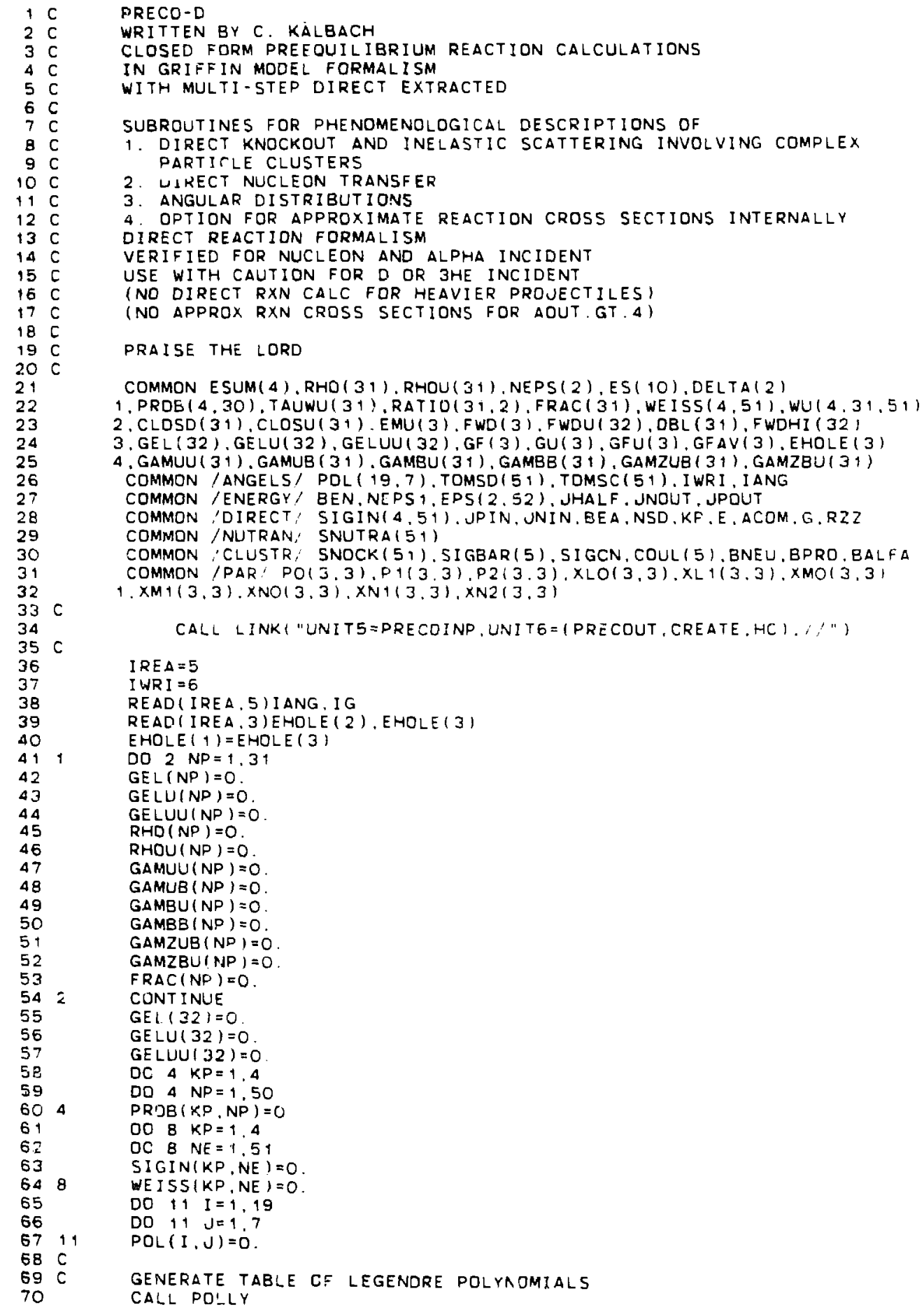




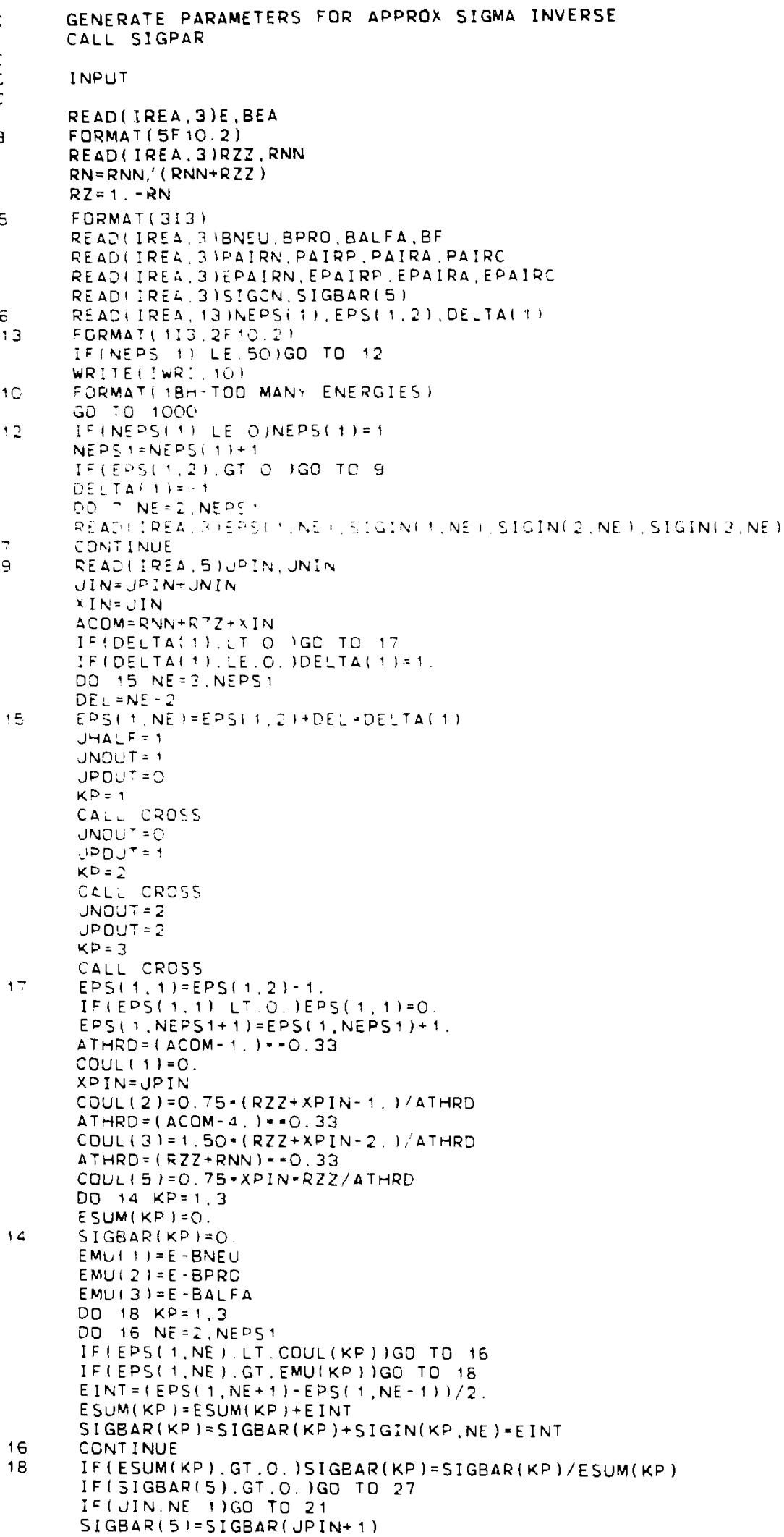




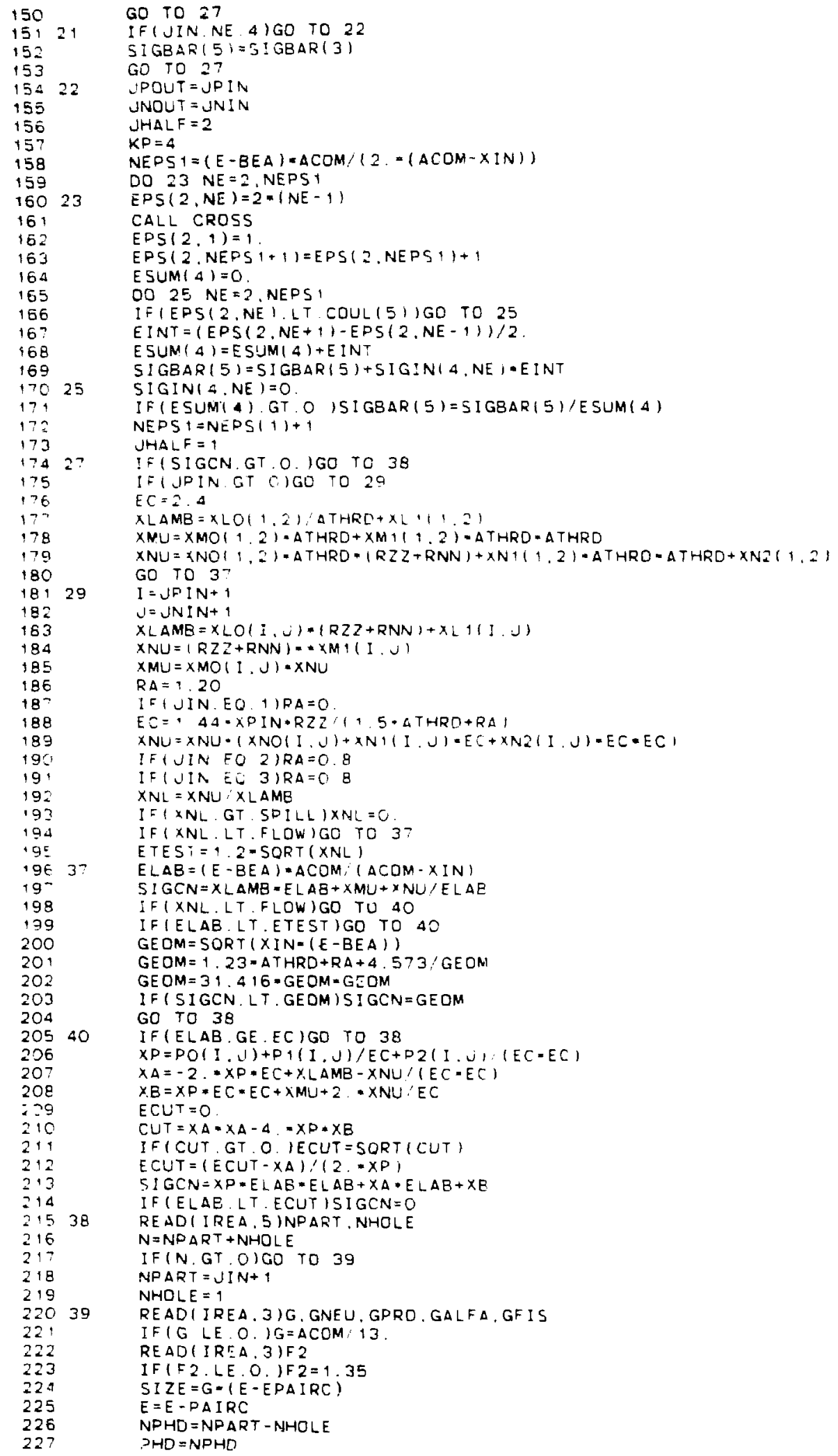




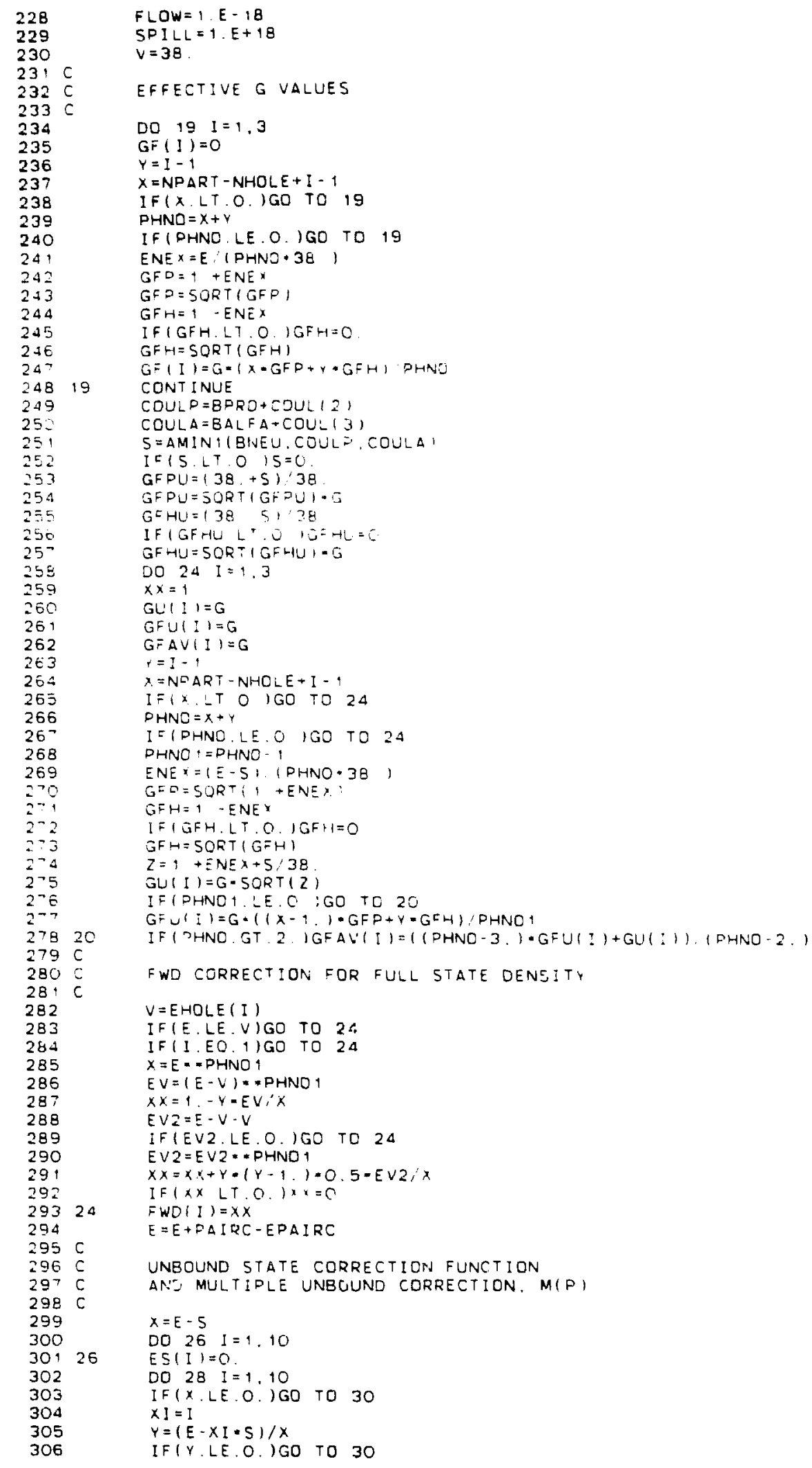




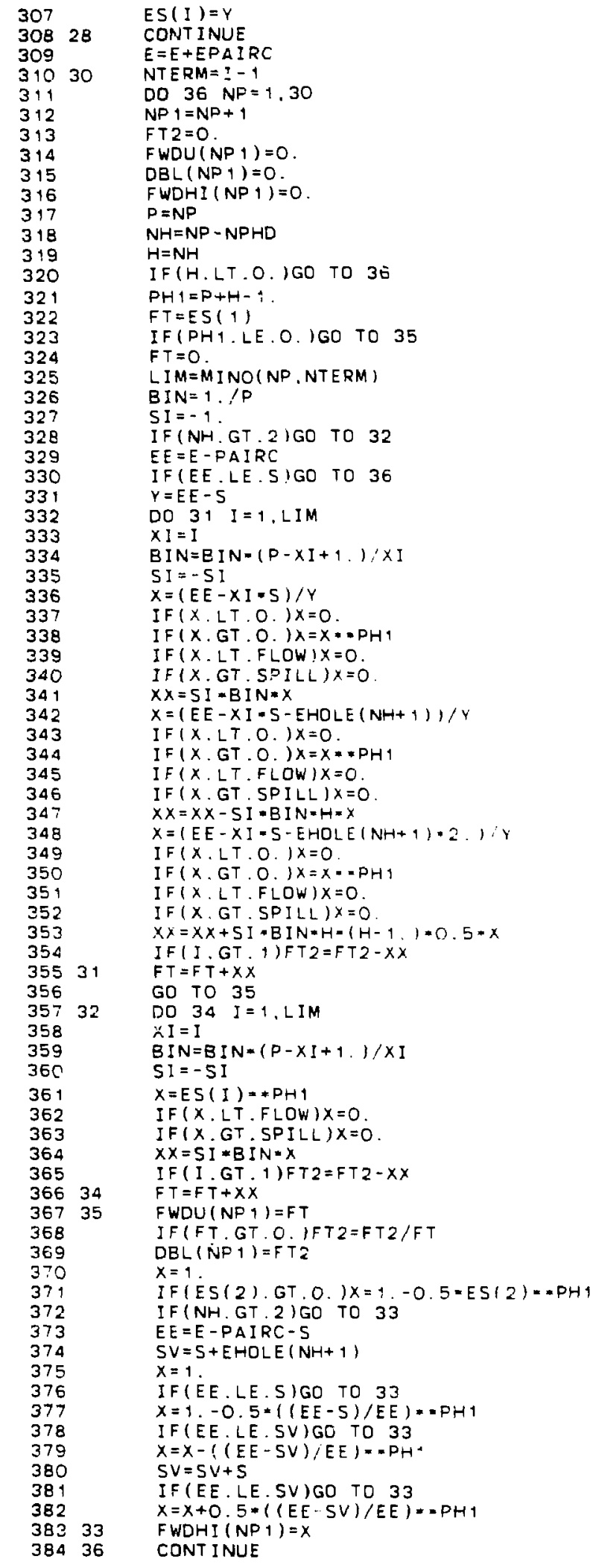




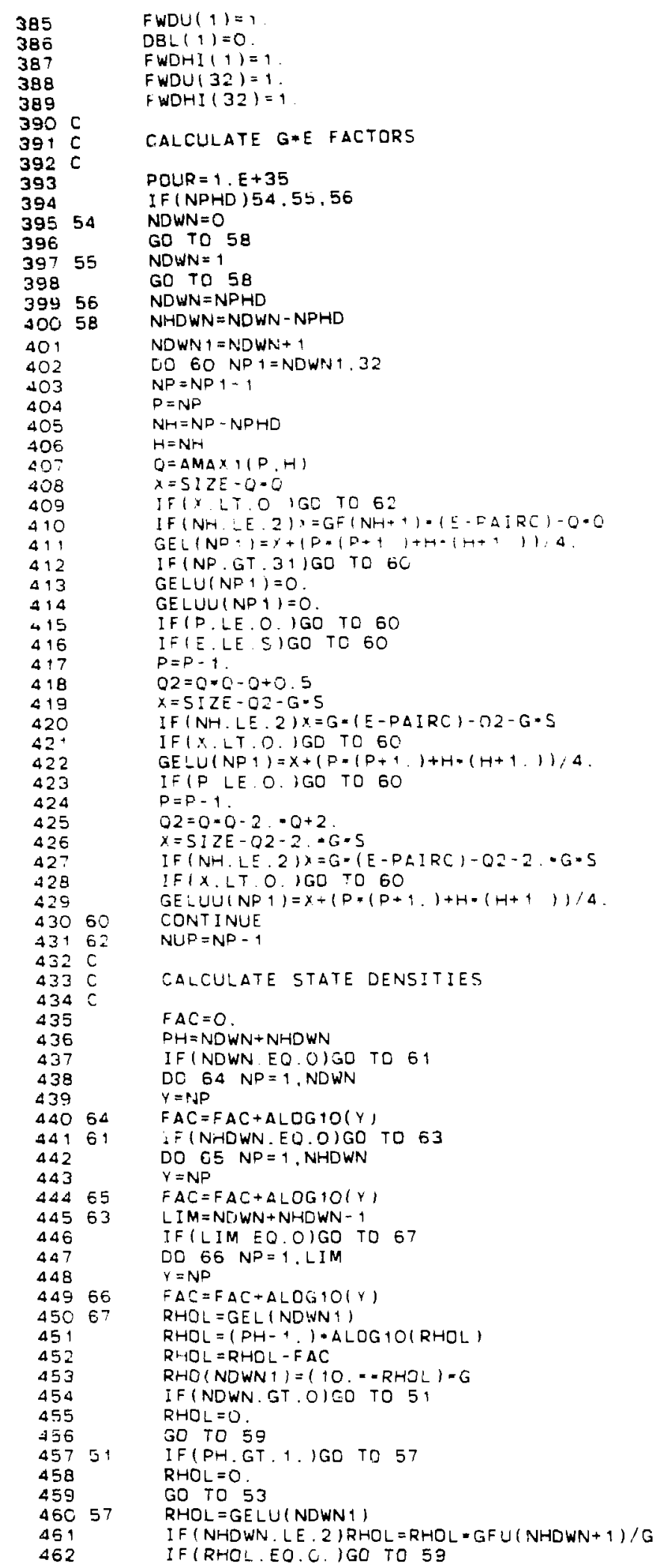




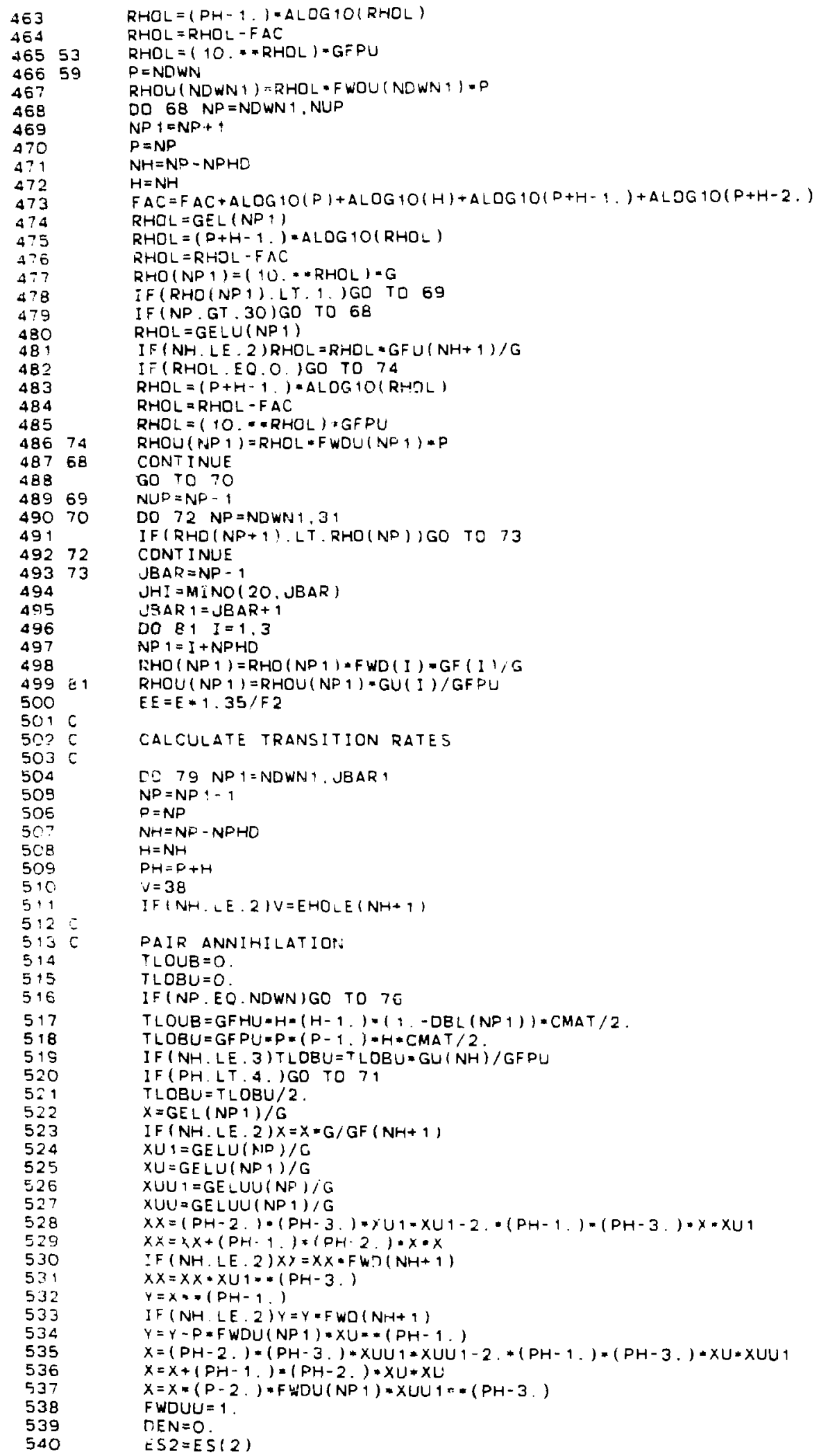




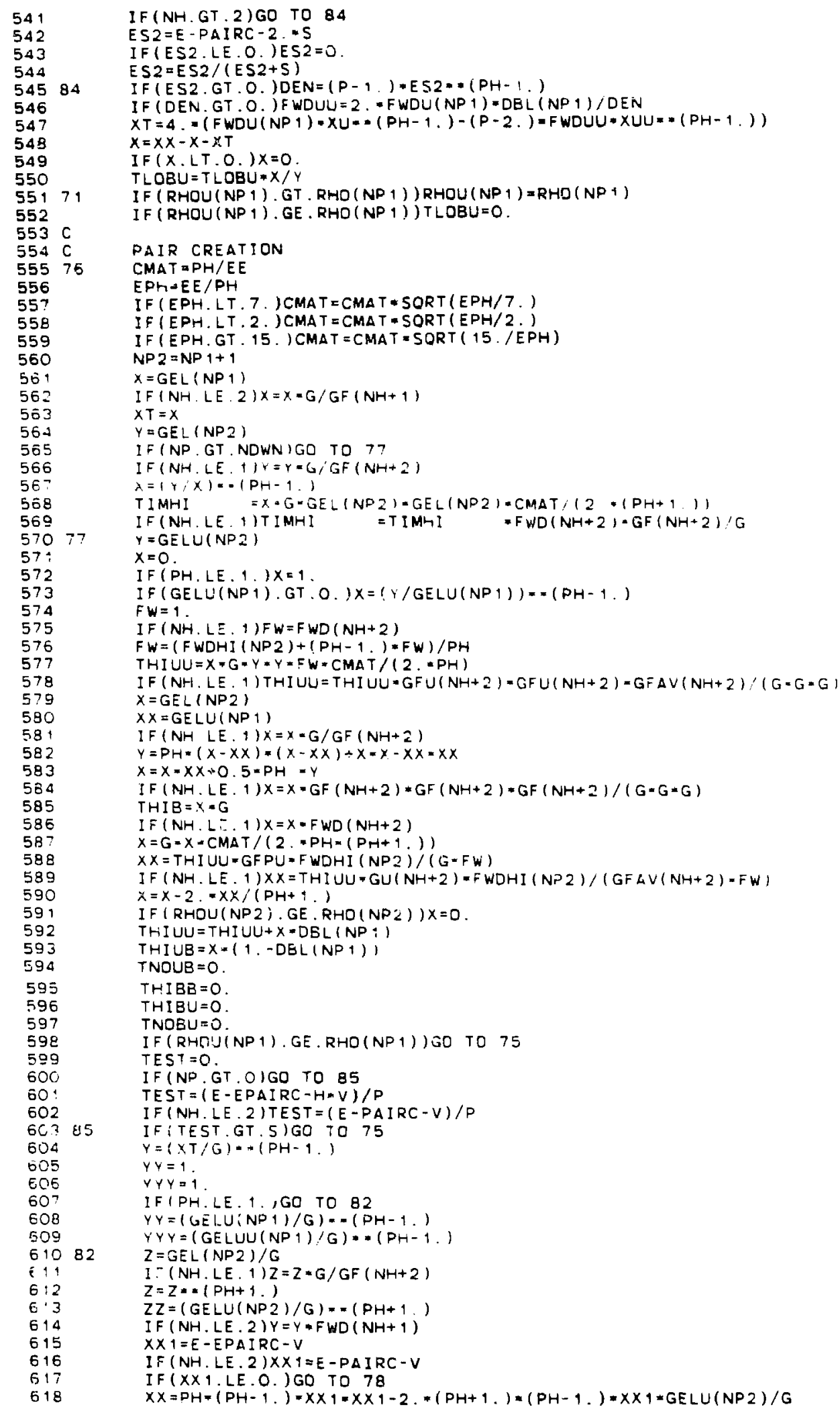




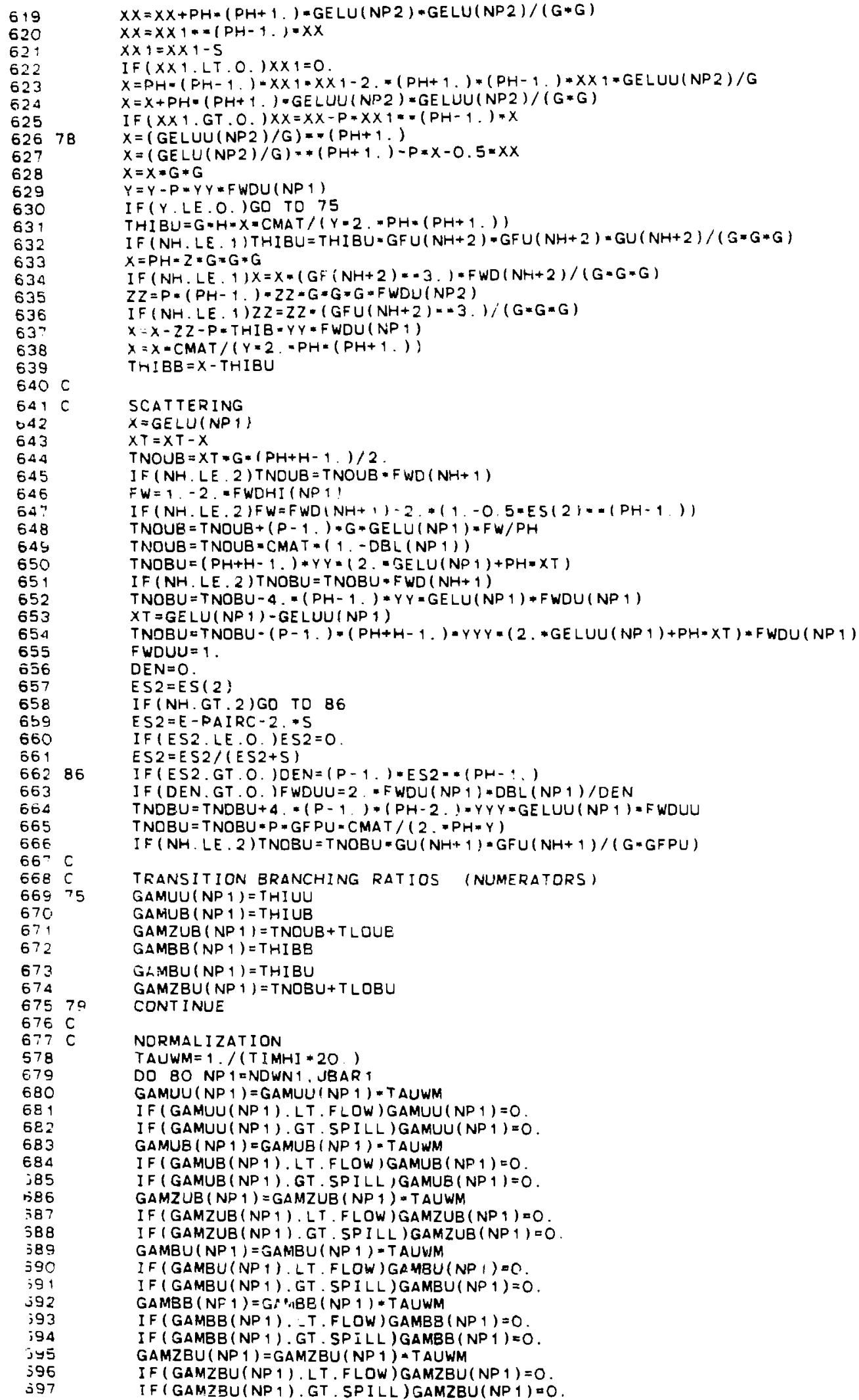




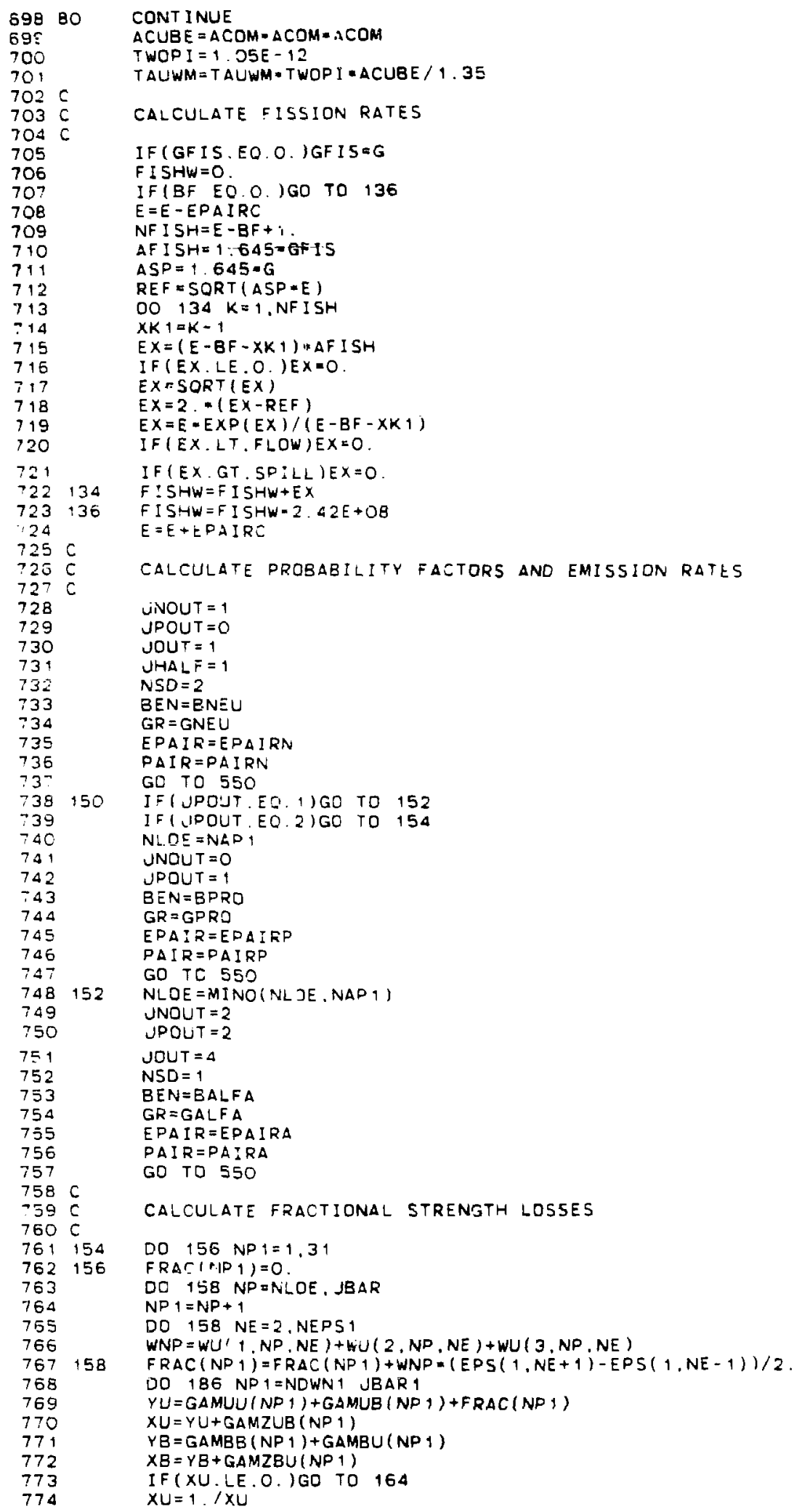

CONT I NUE 


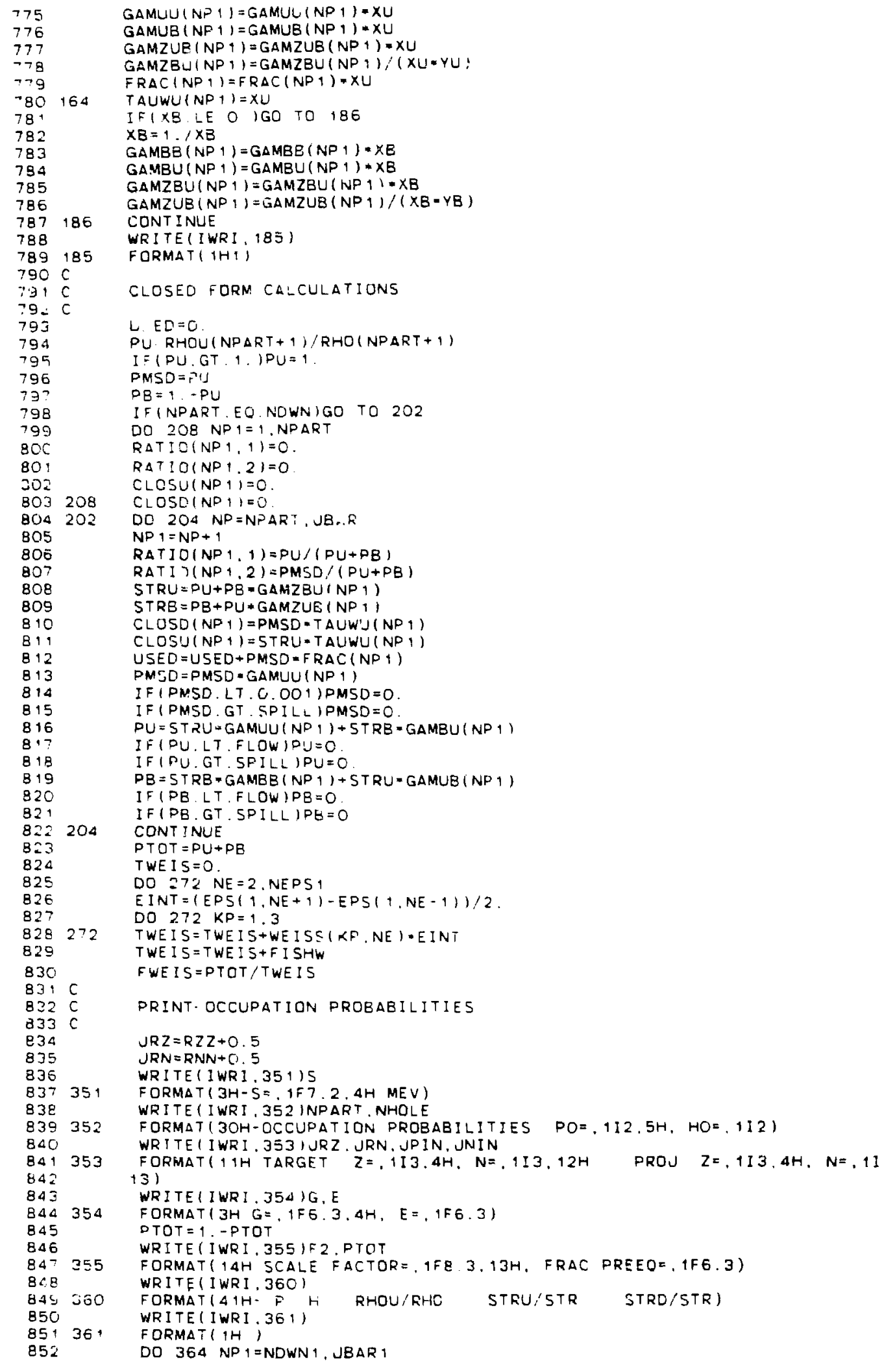




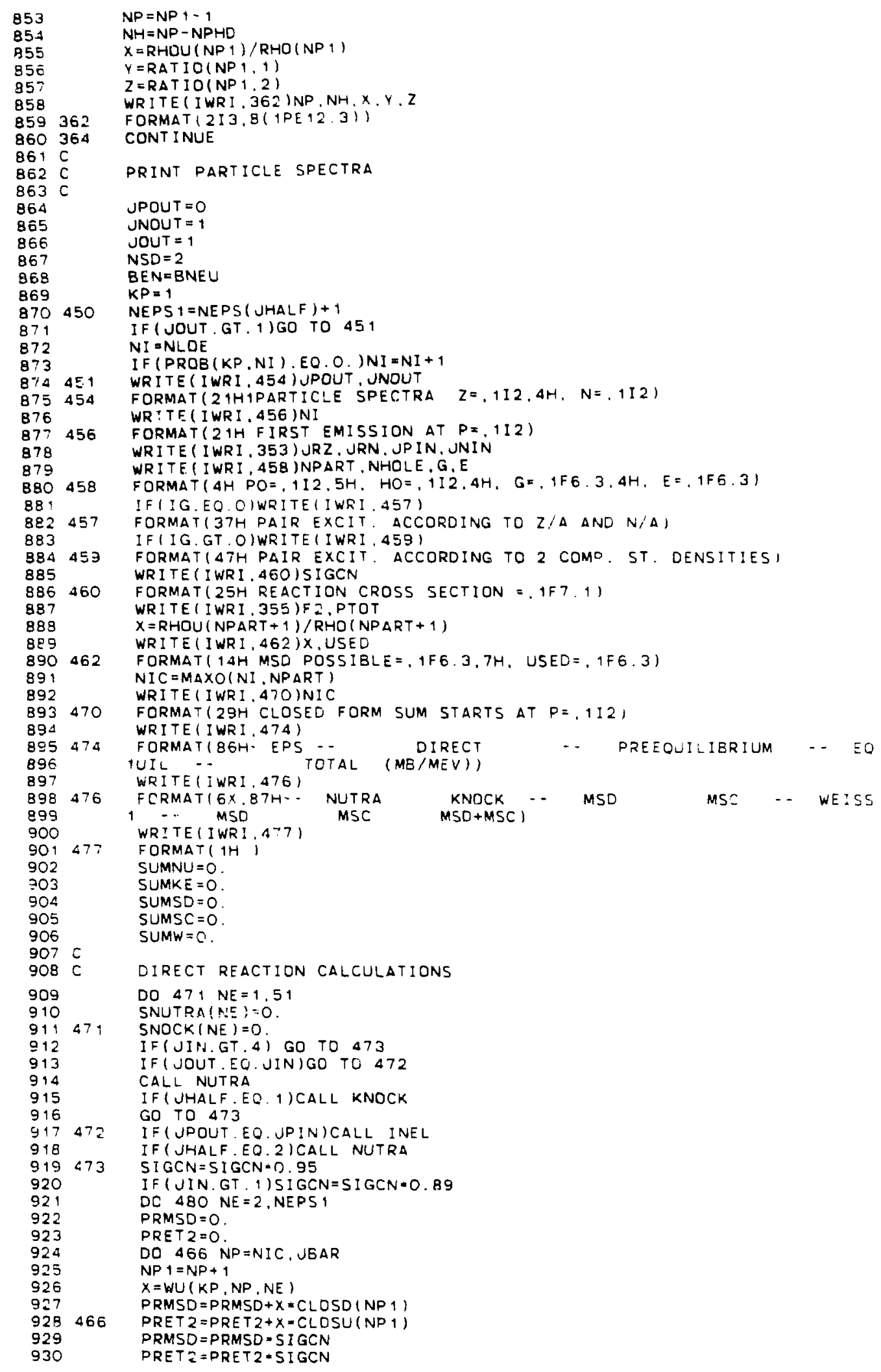




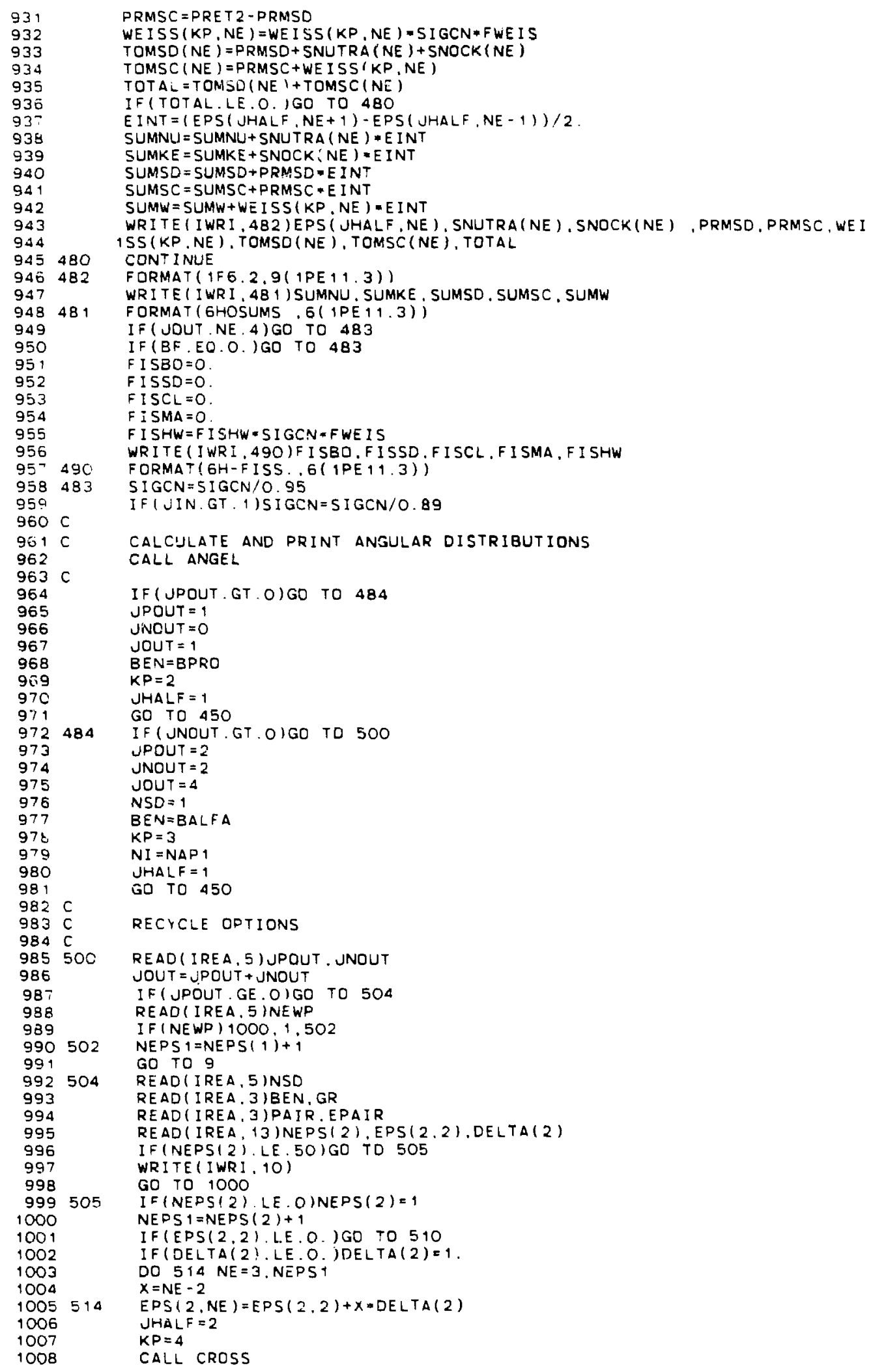




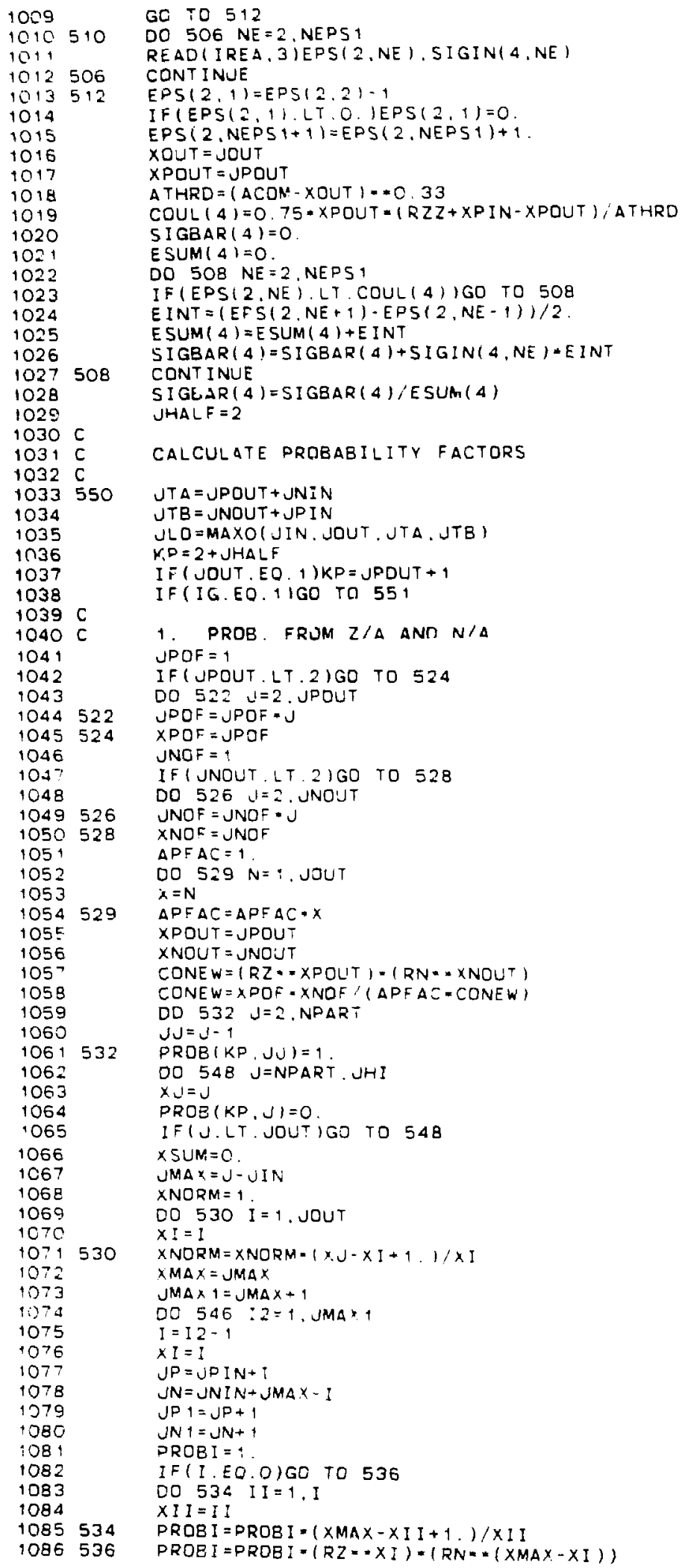




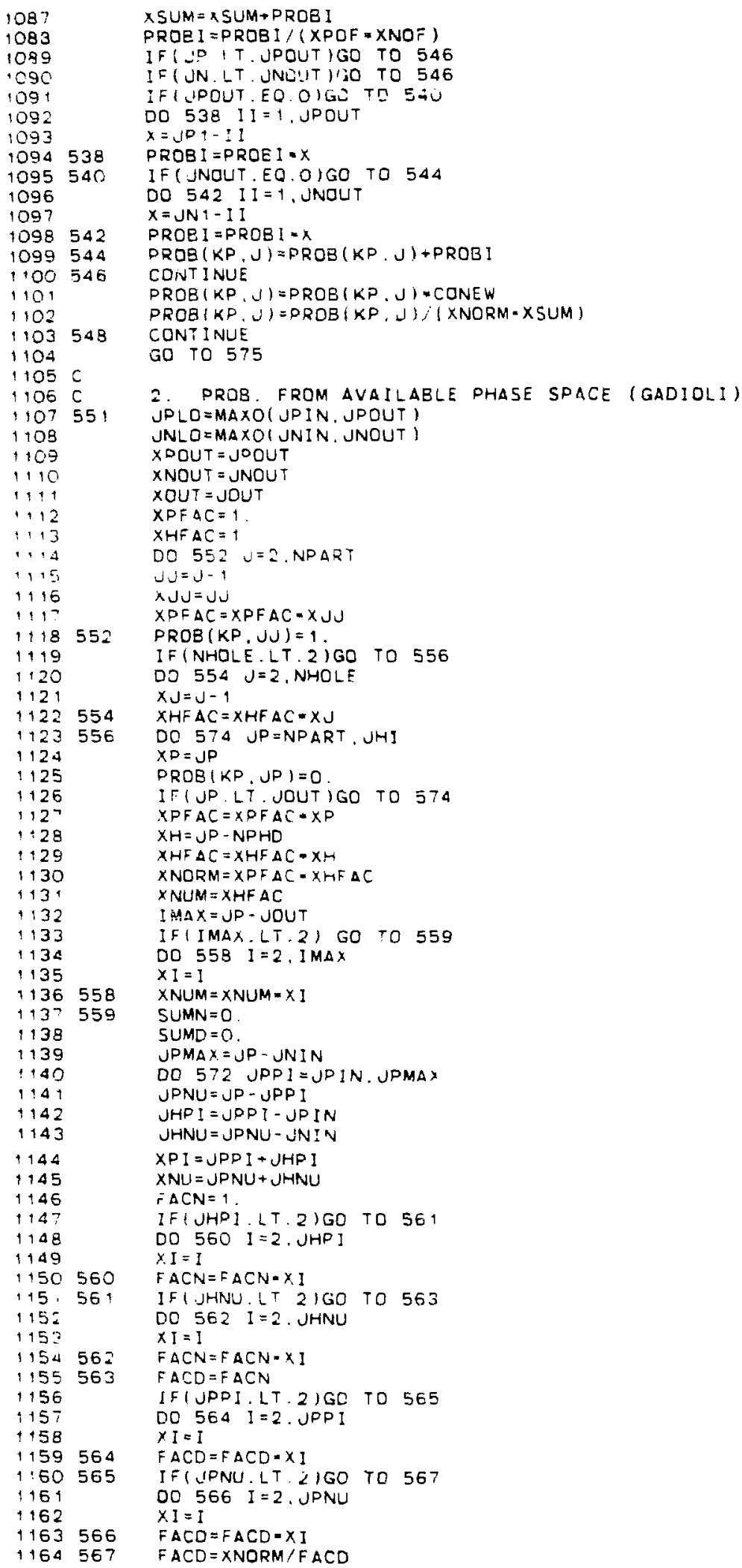




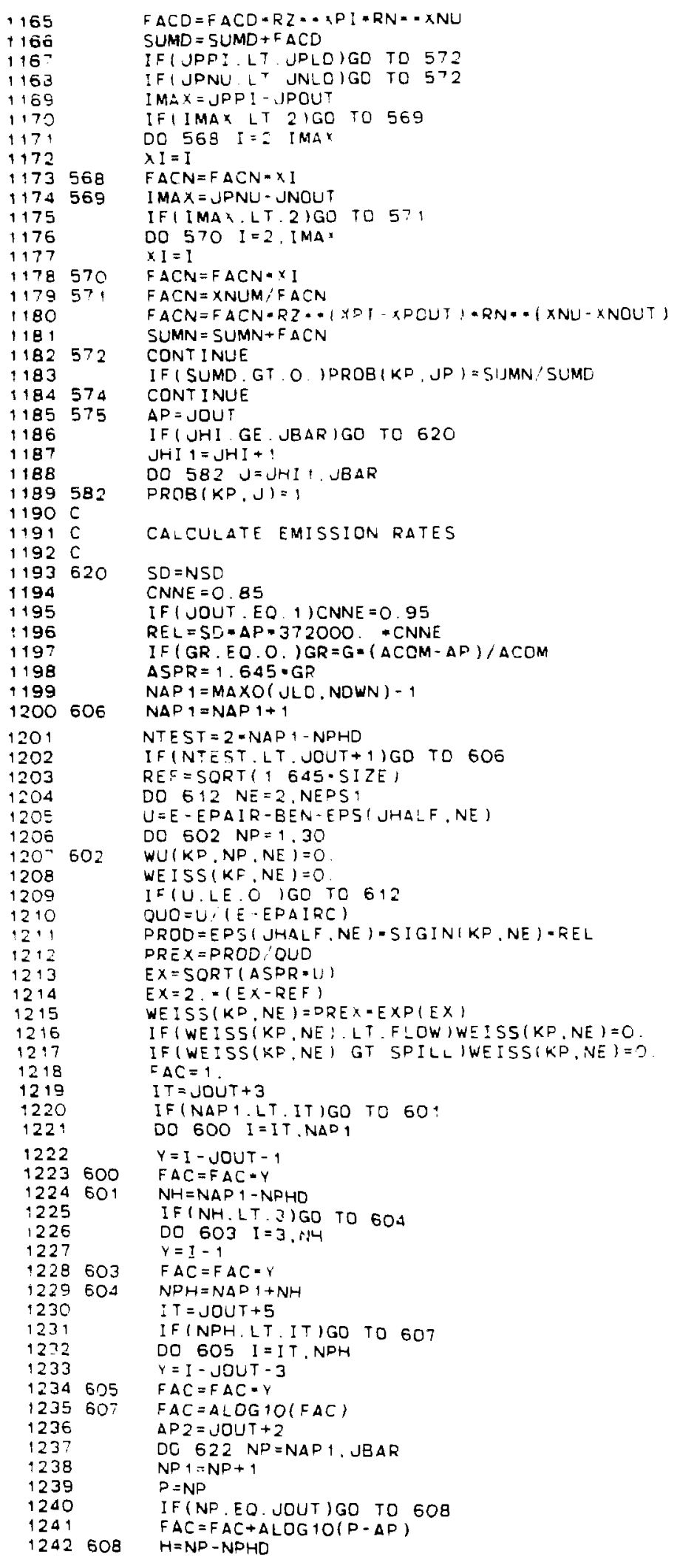




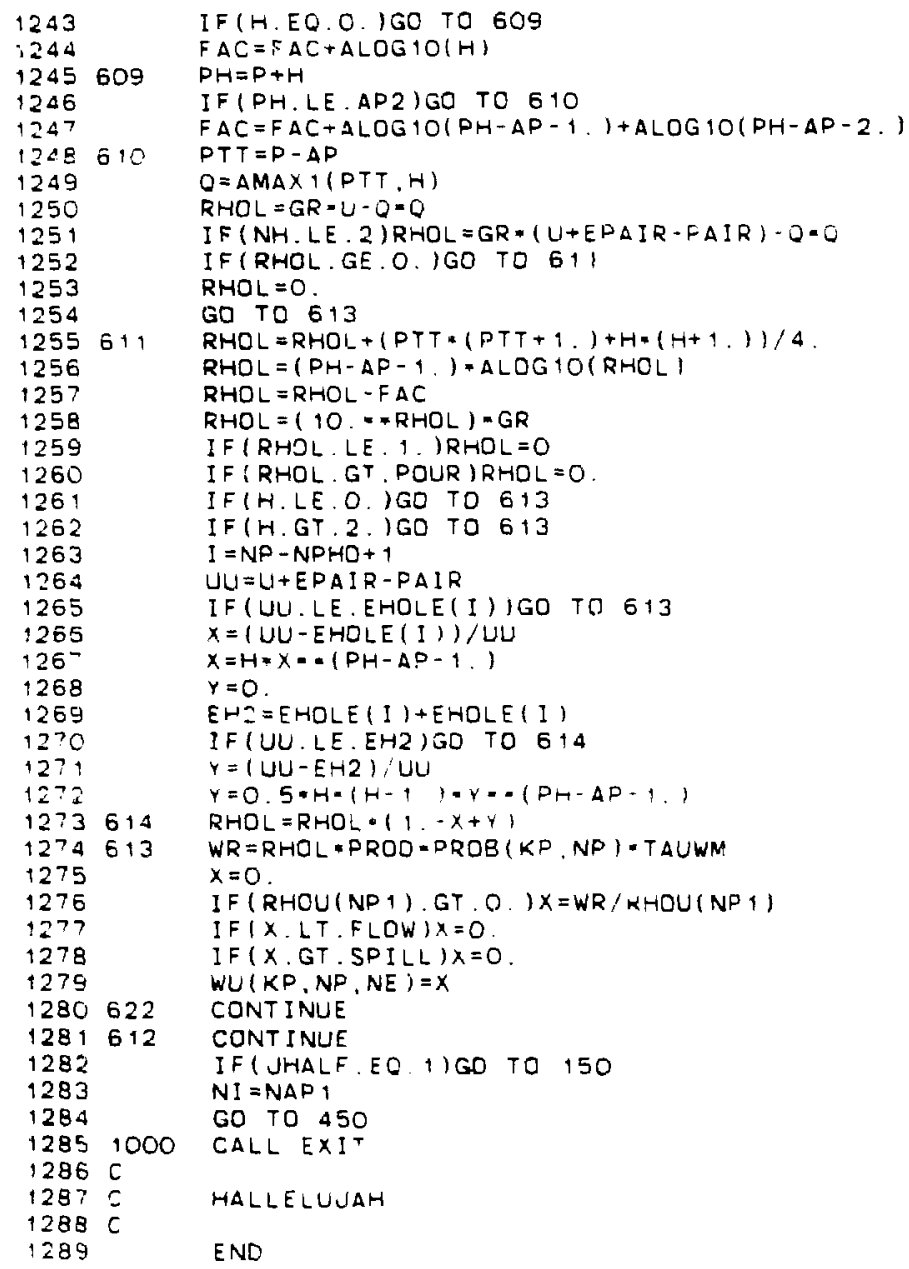




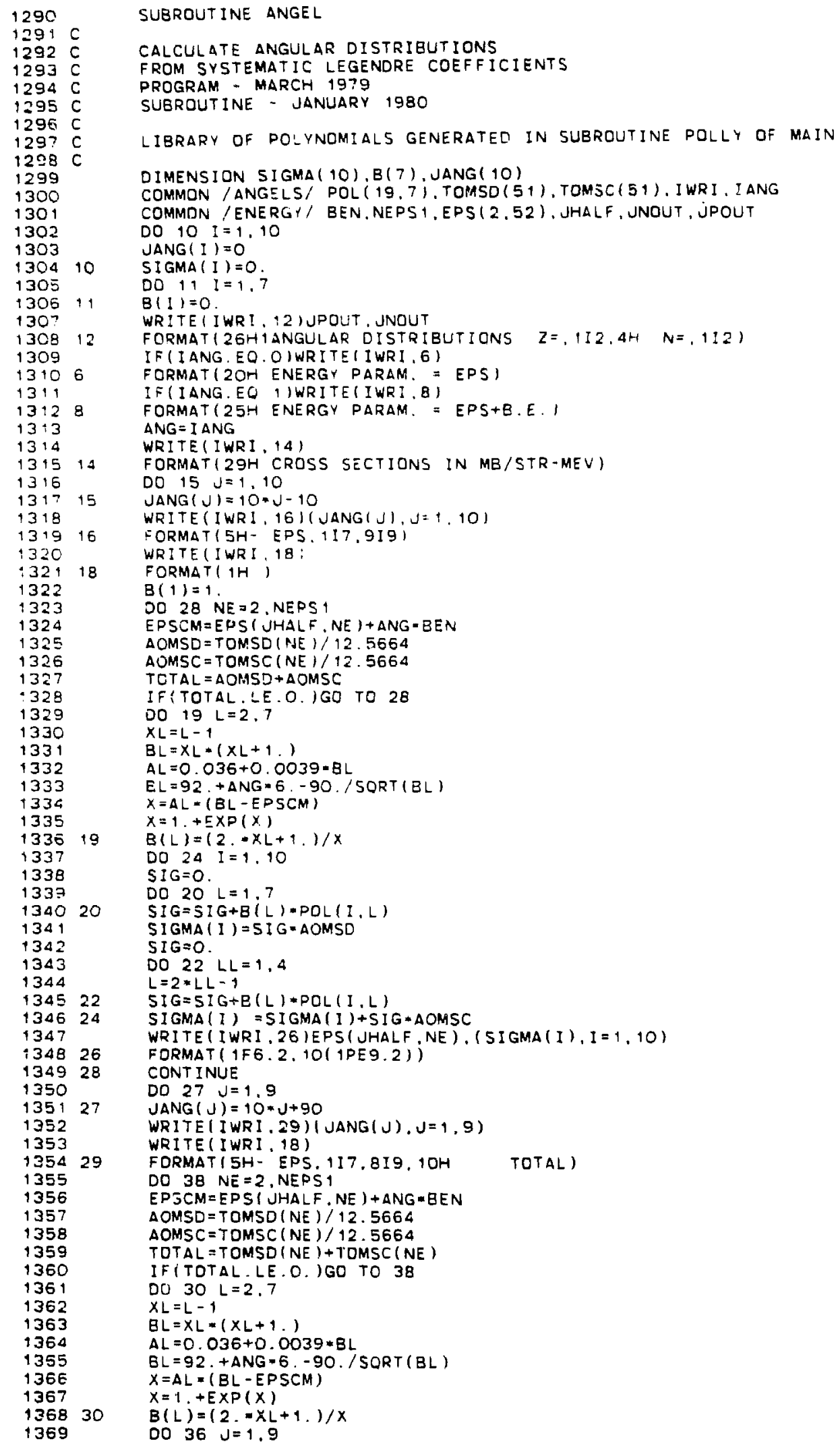




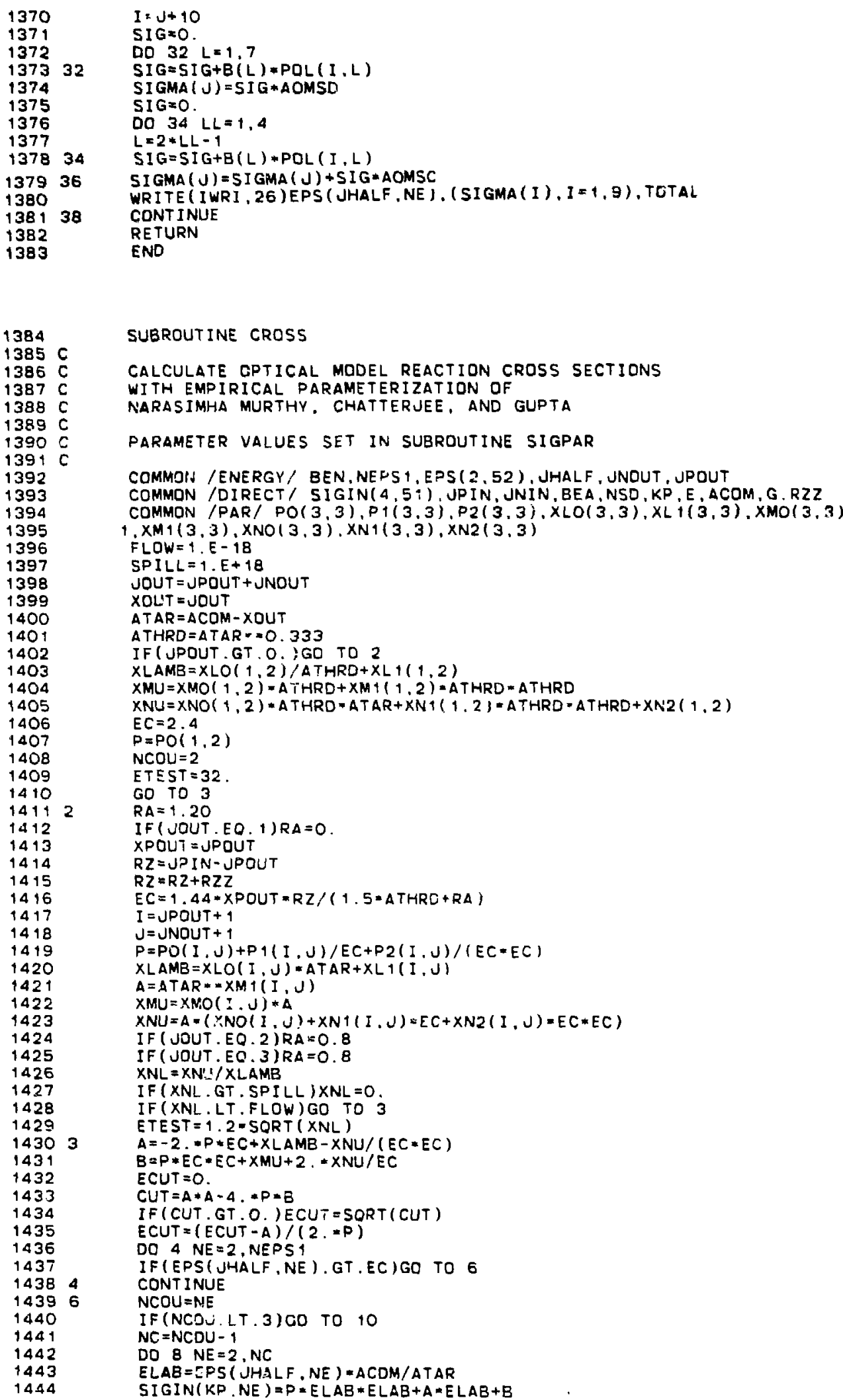

1400

1401

1402

1403

1404

1405

1406

1407

1408

1409

1410

$141+2$

1412

1413

1414

1415

1416

1417

1418

1419

1420

1421

1422

1423

1424

1425

1425

1427

1428

1429

14303

1431

$+432$

1433

1434

1435

1436

1437

14384

14396

1440

1441

1442

1443

1444

SUBROUTINE CROSS

CALCULATE GPTICAL MODEL REACTION CROSS SECTIONS

WI TH EMPIRICAL PARAMETERIZATION OF

NARASIMHA MURTHY, CHATTERJEE, AND GUPTA

PARAMETER VALUES SET IN SUBROUTINE SIGPAR

COMMOH /ENERGY / BEN, NEPS 1, EPS $(2,52)$, JHALF, JNOUT, JPOUT

COMMON /DIRECT/ SIGIN(4,5;), JPIN, JNIN, BEA,NSD, KP, E, ACOM, G, RZZ

COMMON /PAR/PO(3,3),P1(3,3),P2 (3,3),XLO(3,3),XLi(3,3),XMO(3,3)

$1, X M 1(3,3), X N O(3,3), X N 1(3,3), X N 2(3,3)$

$F L O W=1 . E-18$

SPILL $=1 . E+18$

JOUT $=$ JPOUT + JNOUT

XOL'T $=$ JOUT

$\triangle T A R=A C D M-X D U T$

$A T H R D=A T A R=0.333$

IF ( UPOUT.GT.O. :GO TO 2

$X L A M B=X L O(1,2) / \triangle T H R D+X L 1(1,2)$

$X M U=X M O(1,2)=A T H R D+X M+(1,2)=A T H R D=A T H R D$

$X N U=X N O(1,2) * A T H R D=A T A R+X N+(1,2)=A T H R D=A T H R D+X N 2(1,2)$

$E C=2.4$

$P=P O(1,2)$

NCOU $=2$

ETEST $=32$

GO TO 3

$R A=1.20$

IF ( JOUT $E O, 1) R A=0$.

XPOUT $=$ JPOUT

$R Z=J P I N-J P O U T$

$R Z=R Z+R Z Z$

$E C=1.44 * X P O U T=R Z /(1.5 * A T H R D+R A)$

$I=J P O U T+1$

$\mathrm{J}=\mathrm{JNOUT}+1$

$P=P O(I, J)+P 1(I, J) / E C+P 2(I, J) /(E C * E C)$

$X L A M B=X L O(I, U) * A T A R+X L 1(I, J)$

$A=A T A R=X M 1(I, J)$

$X M U=X M O(I . J) * A$

$X N U=A=(X N O(I, U)+X N 1(I, J)=E C+X N 2(I, J)=E C * E C)$

IF ( UDUT. EQ . 2)RA $=0.8$

IF ( JOUT. EO. 3 ) RA $=0.8$

$X N L=X N:-1 / X L A M B$

IF ( XNL.GT. SPI LL IXNL $=0$.

IF (XNL. LT. FLOW) GO TO 3

ETEST $=1.2-S O R T(X N L)$

$A=-2 . P * E C+X L A M B-X N U /(E C * E C)$

$B=P * E C * E C+X M U+2 . X N U / E C$

$E C U T=0$.

$C U T=A * A-4, P \approx B$

IF (CUT. GT .O. )ECUT $=$ SORT (CUT)

$E C U T=(E C U T-A) /(2, * P)$

DO 4 NE $=2$, NEPS 1

IF(EPS (JHALF, NE) . GT ,EC)GO TO 6 CONT INUE

NCOU = NE

IF (NCOU.LT. 3 ) CO TO 10

$N C=N C D U-1$

$D O B N E=2, N C$

$E L A B=C P S(J H A L F, N E)=A C D M / A T A R$

$S I G I N(K P . N E)=P=E L A B * E L A B+A=E L A B+B$ 


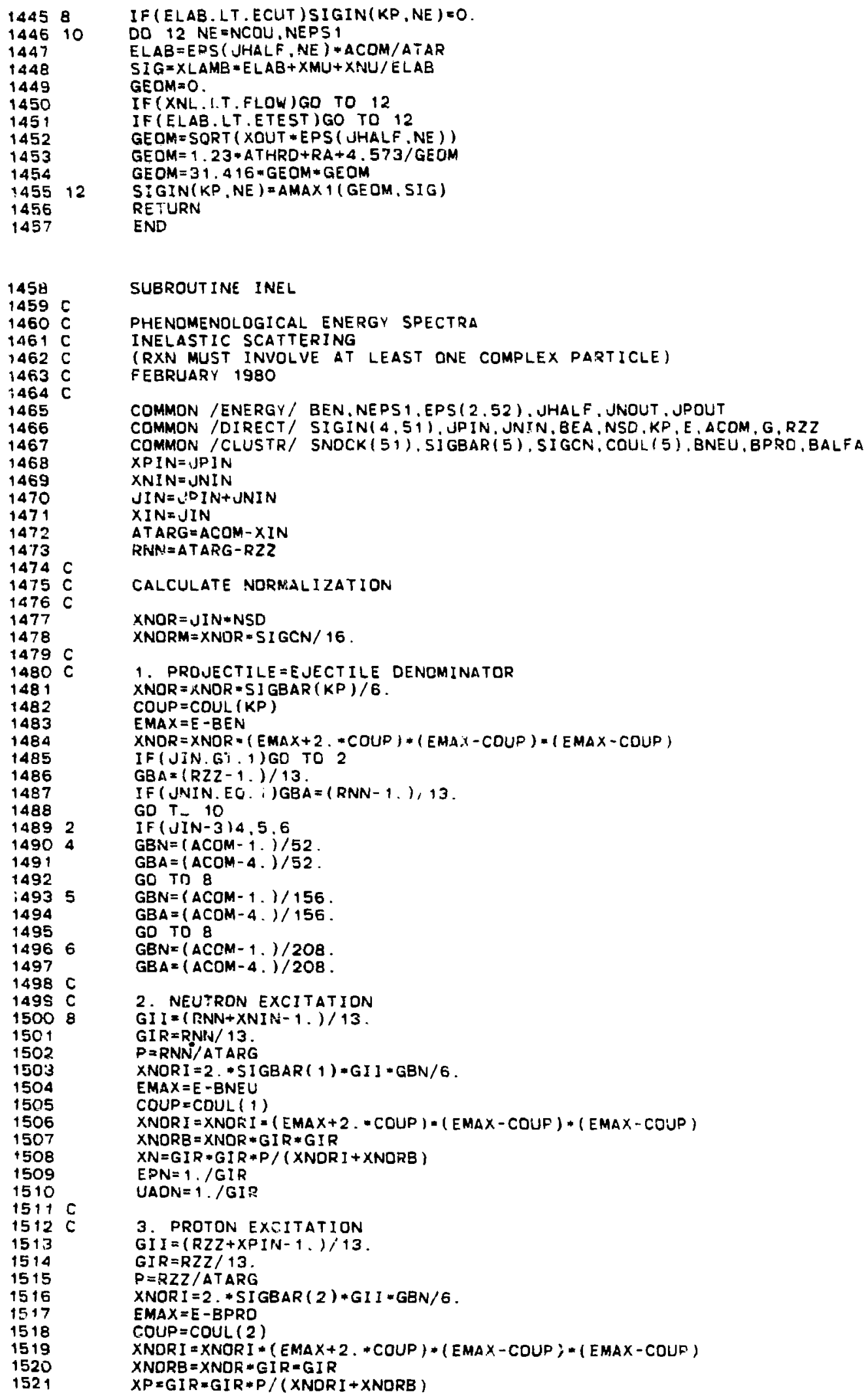

SUBROUTINE INEL

PHENOMENOLOGICAL ENERGY SPECTRA

INELASTIC SCATTERING

(RXN MUST INVOLVE AT LEAST ONE COMPLEX PARTICLE)

FEBRUARY 1980

COMMON /ENERGY/ BEN. NEPS 1.EPS(2.52). JHALF, JNOUT , JPOUT

COMMON /DIRECT/ SIGIN(4,51) JPIN, JNIN, BEA, NSO, KP, E, ACOM, G, RZZ

COMMON /CLUSTR/ SNOCK(51), SIGBAR(5), SIGCN, COUL(5).BNEU,BPRO,BALFA

$X P I N=J P] N$

$X N I N=J N I N$

$J I N=J O I N+J N I N$

$X I N=J I N$

$\triangle T A R G=A C O M-X I N$

RNN $=A T A R G-R Z 2$

CALCULATE NORMALIZATION

$X N O R=J I N * N S D$

$X N O R M=X N O R=S I G C N / 16$.

1. PROJECTILE = EJECT I LE DENOMINATOR

$X N O R=X N O R=S]$ GBAR $(K P) / 6$

COUP $=$ COUL $(K P)$

EMAX $=E-B E N$

$X N O R=X N O R=(E M A X+2 *$ COUP $) *(E M A X-C O U P)-(E M A X-C O U P)$

IF (JIN.G'T. I) GO TO 2

$G B A=(R Z Z-1) /$,

IF ( JNIN.EO. $;) G B A=($ RNN-1, $), 13$.

GO T -10

IF ( JIN-3) $4,5,6$

$G B N=(A C O M-1) /$.

$\mathrm{GBA}=(\mathrm{ACOM}-4), / 52$.

GO TO 8

GBN $=(A C O M-1) /$.156 .

$G B A=(A C O M-4) / 156$.

GD TO 8

$G B N=(A C C M-1) /$.208 .

$G B A=(A C O M-4) /$.208 .

2. NEUTRON EXCITATION

GII $=(R N N+X N I N-1) /$.

GIRIRNN/ 13

$P=R N N / A T$ ARG

XNORI $=2$. $* \operatorname{SIGBAR}(1)=\mathrm{GI}]=\mathrm{GBN} / 6$.

$E M A X=E-B N E U$

COUP $=$ COUL (1)

$X N D R I=X N O F I=(E M A X+2 \cdot-\operatorname{COUP}) \cdot(E M A X-C O U F) *(E M A X-C O U P)$

$X N O R B=X N O R * G I R * G I R$

$X N=G I R * G I R * P /(X N D R I+X N O R B)$

$E P N=1$. $/ G I R$

UADN $=1$. $/$ GIR

3. PROTON EXEITATION

$G I I=(R Z Z+X P I N-1) /$,

$G I R=R Z Z / 13$

$P=R Z Z / A T A R G$

$X N O R I=2 . * S I G B A R(2) * G I I * G B N / 6$.

EMAX $=E-B P R D$

COUP $=$ COUL ( 2 )

$X N O R I=X N O R I *(E M A X+2 . *$ COUP $) *(E M A X-C O U P) *(E M A X-C O U F)$

$X N O R B=X N O R=G I R=G I R$

$X P=G I R=G I R * P /(X N D R I+X N O R B)$ 


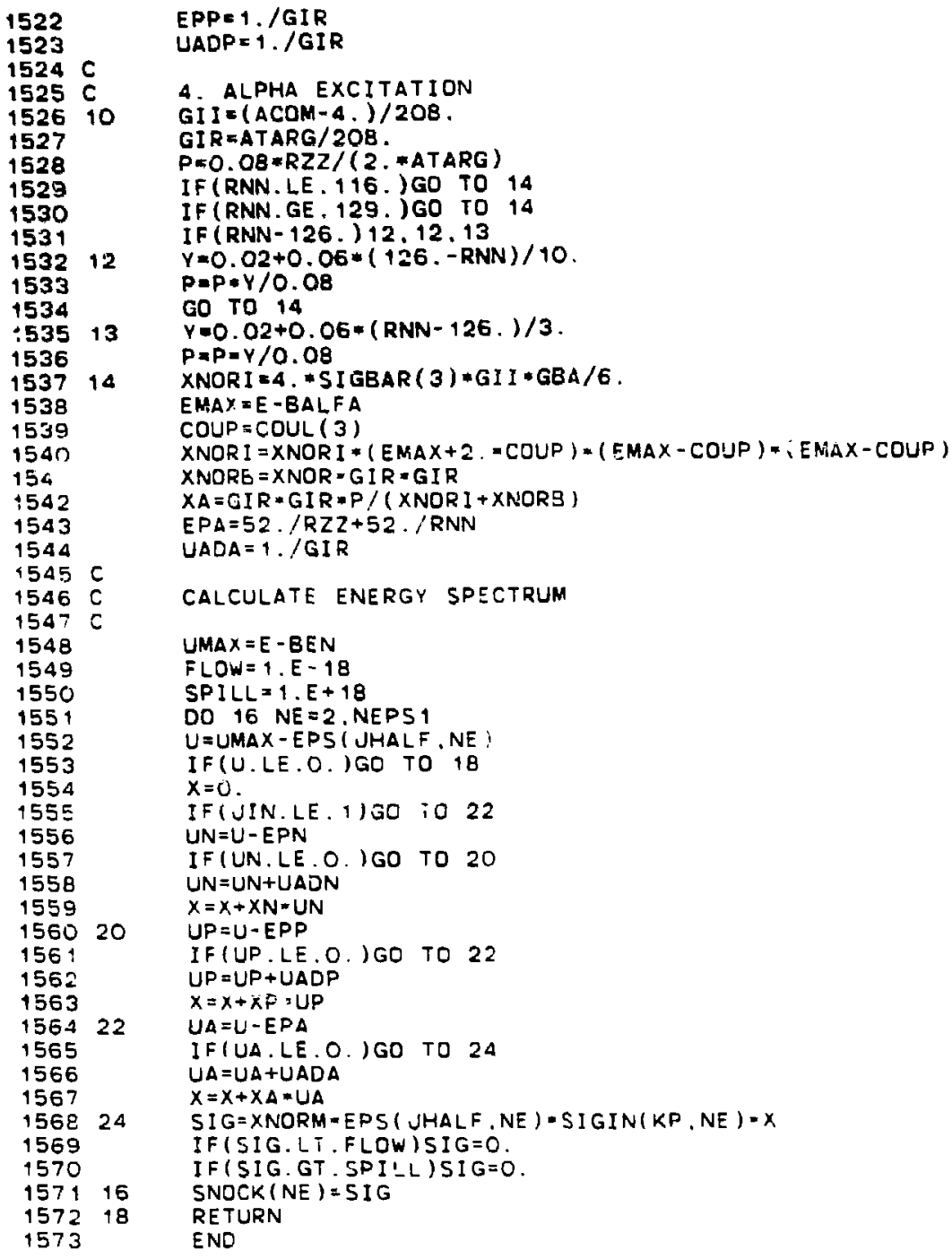

4. ALPHA EXCITATION

GII $=(A C O M-4) /$.208 .

GIR=ATARG/208.

P $=0.08 * R Z Z /(2 . * A T A R G)$

IF (RNN. LE. 116.) GO TO 14

IF (RNN. GE, 129.) GO TO 14

IF $($ RNN-126. $) 12,12.13$

$Y=0.02+0.06 *(126 .-R N N) / 10$.

$P=P=Y / O .08$

GO TO 14

$Y=0.02+0.06 *(R N N-126) /$.3 .

$P=P=Y / 0.08$

$X N O R I=4$, $\$ I$ GBAR ( 3$) * G I I * G B A / 6$.

EMAX $=E-B A L F A$

COUP $=$ COUL ( 3 )

$X N O R I=X N O R I *(E M A X+2=$ COUP $) *($ EMAX - COUP $)=i E M A X-$ COUP $)$

$X N D R E=X N O R=G I R=G I R$

$X A=G I R * G I R * P /(X N O R I+X N O R B)$

$E P A=52 . / R Z Z+52 . / R N N$

UADA $=1 . / G I R$

CALCULATE ENERGY SPECTRUM

UMAX $=E-B E N$

$F L O W=1 . E-18$

SPILL $=1 . E+18$

DO 16 NE = 2. NEPS 1

$U=U M A X-E P S$ ( JHALF . NE :

IF (U.LE.O.)GO TO 18

$\mathrm{X}=0$.

IF(UIN.LE. I)GO iO 22

$U N=U-E P N$

IFIUN. LE. O. IGO TO 20

$U N=U N+U A D N$

$X=X+X N-U N$

$U P=U-E P P$

IF (UP.LE,O.)GO TO 22

$U P=\cup P+U A D P$

$X=X+X F=U P$

$U A=U-E P A$

IFIUA.LE.O.)GO TO 24

$\cup A=U A+U A D A$

$X=X+X \triangle * \cup A$

$S I G=X N O R M-E P S(J H A L F, N E)=S I G I N(K P, N E)=X$

IF(SIG. LI T FLOW)SIG $=0$.

IF(SIG.GT. SPI'LL)SIG $=0$

SNOCK $(N E)=S I G$

RETURN

END

1574
$1575 C$
1576
1577
1578
1579
1580
1581
1582
1583
1584
1585
1586
1587
1588
1589
1590
1591
1592
1593
1594

\section{SUBROUTINE KNOCK}

PHENOMENOLOGI CAL ENERGY SPECTRA

KNOCKOUT OF N. P, OR ALPHA

(RXN MUST HAVE AT LEAST ONE COMPLEX PARTICLE)

FEBRUARY 1980

COMMON /ENERGY / BEN, NEPS1, EPS $(2,52)$, JHALF, JNOUT, JPOUT

COMMON /OIRECT/ SIGIN(4.51). JPIN. JNIN,BEA, NSD.KP,E, ACOM, G, RZZ

COMMON /CLUSTR/ SNOCK 51 ), SIGEAR(5), SIGCN, COUL (5).BNEU,BPRO, BALFA JIN=JPIN+JNIN

$X I N=J I N$

$X P I N=J P I N$

XNIN $=$ JNIN

JOUT $=$ JPOUT + JNOUT

XOUT $=$ JOUT

$X P O U T=J P O U T$

XNOUT $=$ JNOUT

$\triangle T A K G=A C D M-X I N$

RNN $=A T A R G-R Z Z$

$\triangle R E S=\triangle C O M-X O U T$ 


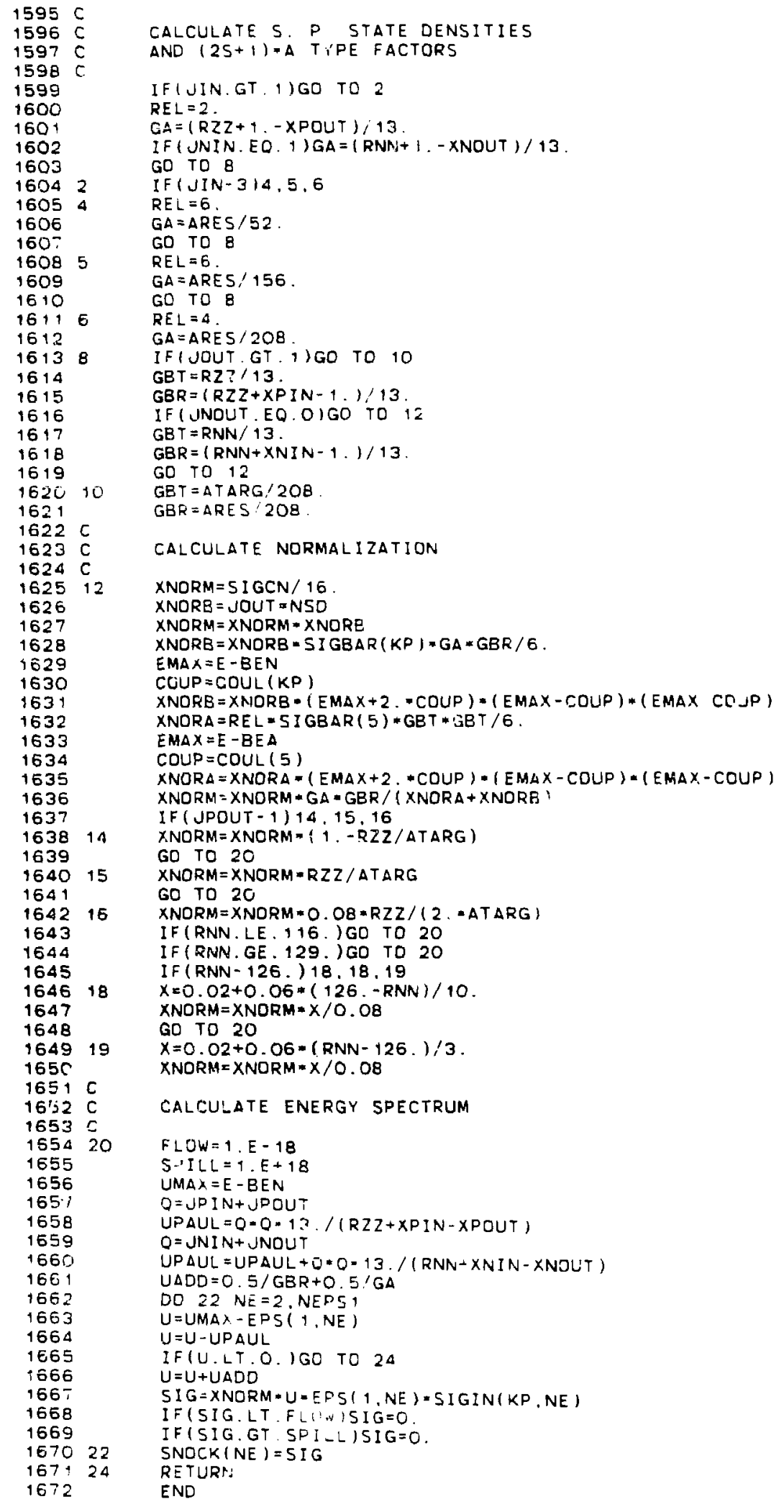




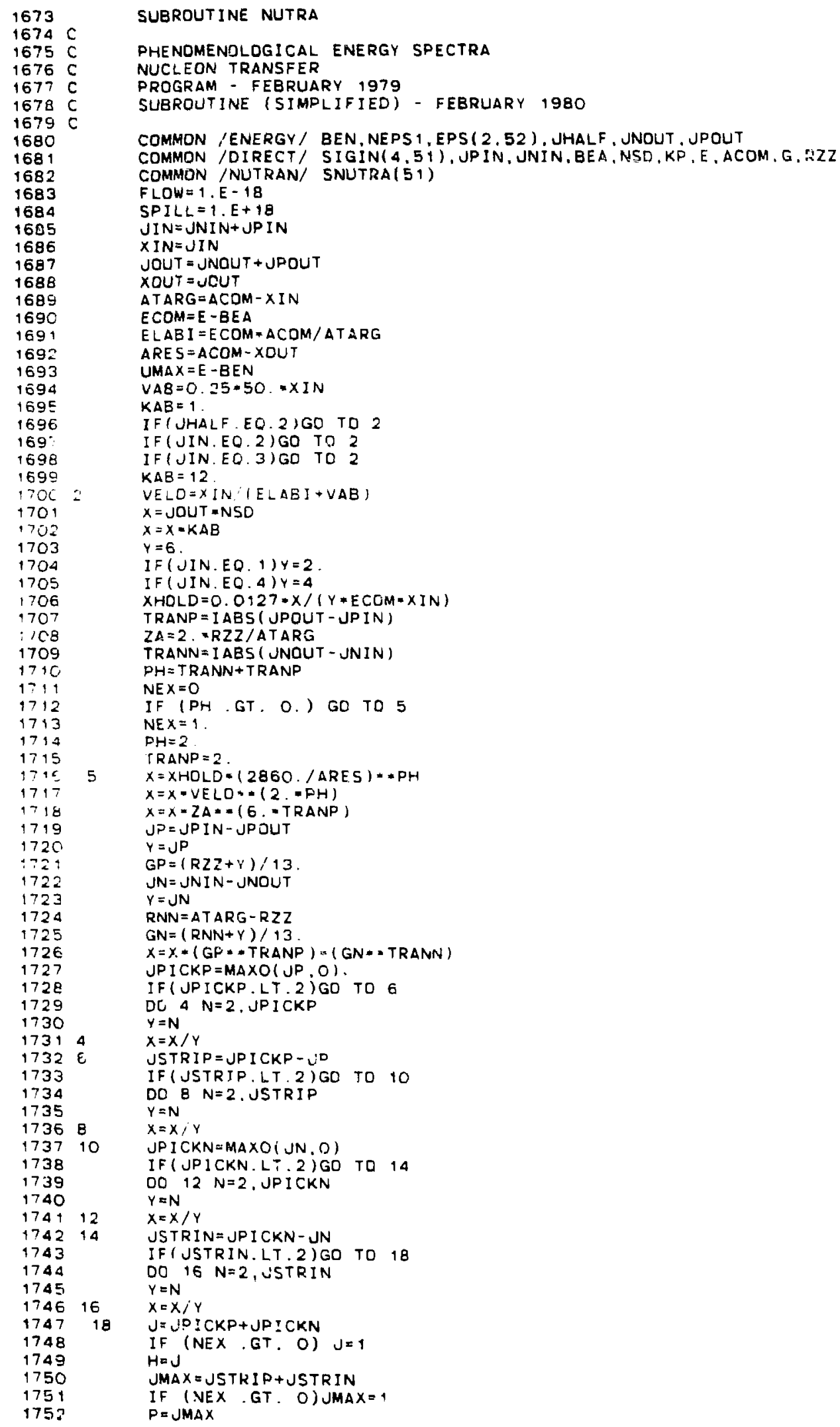

1673

$1674 \mathrm{C}$ $1675 \mathrm{C}$ 1676

$1677 \mathrm{C}$ $1678 \mathrm{C}$ $1679 \mathrm{C}$

1680

1681

1682

1683

1684

1605

1686

1687

1688

1689

1690

1691

1692

1693

1694

1695

1696

$169^{\circ}$

1698

1690

$1706=$

1701

1702

1703

1704

1705

1706

1707

$1 / \mathrm{CB}$

1709

1710

1711

1712

1713

1714

1715

1796

1717

1718

1719

1720

$+721$

1722

1723

1724

1725

1726

1727

1728

1729

1730

17314

$1732 \varepsilon$

1733

1734

1735

1736

$1737 \quad 10$

1738

1739

1740

174912

$1742 \quad 14$

1743

1744

1745

$1746 \quad 16$

1747

1748

1749

1750

1751

1752

SUBROUTINE NUTRA

PHENOMENDLOGICAL ENERGY SPECTRA

NUCLEON TRANSFER

PROGRAM - FEBRUARY 1979

SUBROUTINE (SIMPLIFIED) - FEBRUARY 1980

COMMON /ENERGY/ BEN, NEPS 1,EPS(2.52), JHALF, JNOUT, JPOUT

COMMON /DIRECT/ SIGIN(4.51), JPIN, JNIN, BEA,NSD,KP, E, ACOM, G, RZZ

COMMON/NUTRAN/SNUTRA(51)

$F L O W=1 . E-18$

$S P I L L=1 . E+18$

$J I N=J N I N+J P I N$

$X I N=J I N$

JOUT $=$ JNOUT + JPOUT

XOUT $=$ UDUT

$\triangle T A R G=A C D M-X I N$

$E C O M=E-B E A$

$E L A B I=E C O M=A C D M / A T A R G$

$A R E S=A C O M-X D \cup T$

UMAX $=E-B E N$

$V A B=0.25 \cdot 50 \cdot X I N$

$K A B=1$.

IF( JHALF.EQ.2 IGO TO 2

IF ( JIN.EO. 2)GO TO 2

IF(UIN.EO. 3 )GO TO 2

$K A B=12$

$V E L D=X I N(E L A B I+V A B)$

$x=$ JOUT $=$ NSO

$x=x=K A B$

$y=6$.

IF (UIN.EQ. 1) Y $=2$

IF (JIN.EO. 4$) Y=4$

$X H O L D=0.0127 * X /(Y * E C O M=X I N)$

TRANP = I ABS ( JPOUT - JP IN )

$Z A=2, \approx R Z Z / A T A R G$

TRANN = I ABS ( UNQUT - JNIN)

$P H=T R A N N+T R A N P$

NEX $=0$

IF (PH. GT, O.) GO TO 5

NEX $X=1$.

$\mathrm{PH}=2$

TRANP $=2$

$5 \quad x=x H O L D=(2860, / \triangle R E S) * P H$

$x=x-V E L O=(2 . F H)$

$X=X=Z A=-(6 .-T R A N P)$

$J P=J P I N-J P D U T$

$Y=J P$

$G P=(R Z Z+Y) / 13$

$J N=J N I N-J N O U T$

$Y=J N$

RNN $=A T$ T ARG $-R Z Z$

$G N=(R N N+Y) / 13$

$x=x_{0} *($ GF $*$ TRANP $)=($ GN - TRANN $)$

$J P I C K P=M A X O(J P, O)$

IF (JPICKP.LT. 2 )GO TO 6

DC $4 N=2$. JPI CKP

$Y=N$

$X=X / Y$

JSTRIP = JPI CKP - JD

IF (JSTRIP. LT. 2 )GO TO 10

DO $8 N=2$. JSTRIP

$Y=N$

$X=X ; Y$

$J P I C K N=M A X O(J N, O)$

IF ( JPICKN.LI. 2 )GO TO 14

$O O 12 N=2$, JPICKN

$Y=N$

$X=X / Y$

JSTRIN = JPI CKN - JN

IF (USTRIN.LT.2)GO TO 18

DO $16 \mathrm{~N}=2$, USTRIN

$Y=N$

$X=X ; Y$

$J=J P ! C K P+J P I C K N$

IF (NEX . GT. O) $J=1$

$H=J$

JMAX = JSTRIP+JSTRIN

IF (NEX .GT. O) JMAX $=1$

$P=J M A X$ 


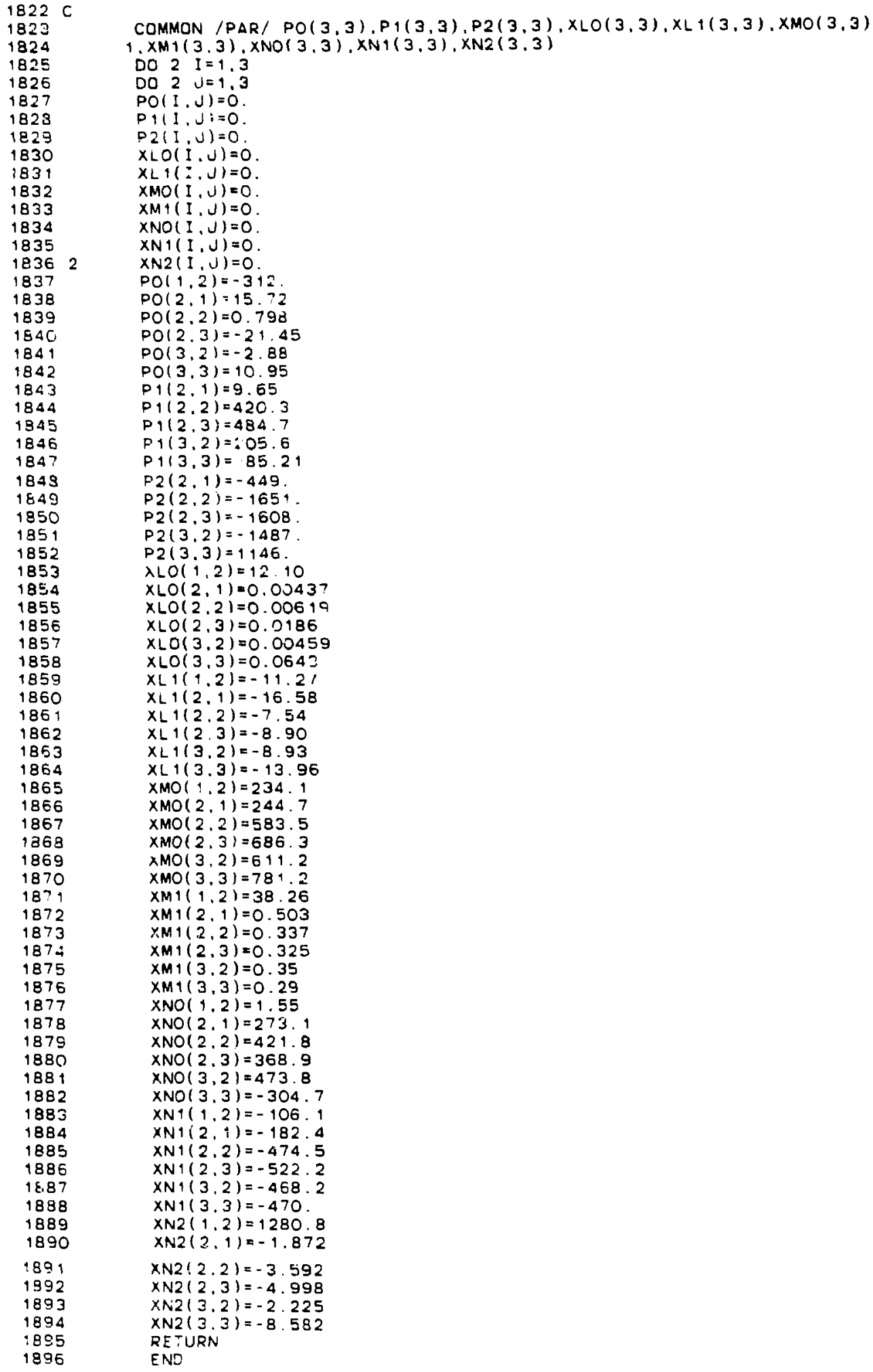




\section{APPENDIX B}

\section{SAMPLE INPUT}

1

$38.00 \quad 38.00$

$26.00 \quad 28.00$

$14.08 \quad 5.05$

16

966.00

\begin{tabular}{ll}
$1.00 \quad 740.00$ \\
\hline
\end{tabular}

2.00

3.00

4.00

5.00

6.00

7.00

8. 00

9.00

10.00

12.00

14.00

16.00

20.00

24.00
26.00

20

4.23

1.35

$\begin{array}{ll}-1 & 0\end{array}$

10

21

4.23

$-1$

0

$66.00 \quad 5.05$

$26.00 \quad 28.0$

$14.08 \quad 5.05$

0.00

0.00

30

10

$$
2.00 \quad 2.00
$$

0.00

1

0.00

16.21

0.00

$24 \quad 2.00 \quad 2.00$

2

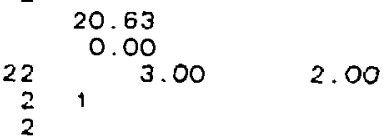

18.24

0.00

$\begin{array}{lr}21 & 0.00 \\ 1 & 6.00 \quad 2.00\end{array}$

0

$20.04 \quad 4.79$

$92.00 \quad 143.00$

$5.69 \quad 4.79$

0.00

0.00

0.00

$24 \quad 1.00$

$\begin{array}{ll}1 & 0 \\ 0 & 0\end{array}$

16.62

0.00

-1
-1

16.57

17.13

a. 20

0.00 $\begin{array}{rl}4.00 & 0.00 \\ 36.00 & 0.00\end{array}$

$123.00 \quad 0.00$

$258.00 \quad 2.00$

$\begin{array}{rr}402.00 & 26.00 \\ 517.00 & 140.00\end{array}$

$596.00 \quad 350.00$

$655.00 \quad 570.00$

$702.00 \quad 730.00$
779.00

$779.00 \quad 980.00$

$834.00 \quad 1170.00$

$881.00 \quad 1300.00$

$932.00 \quad 1420.00$

$\begin{array}{ll}957.00 & 1500.00 \\ 965.00 & 1520.00\end{array}$
17.49

17.97 
$-5=10.45$ MEV

OCCUPATIONE

TARGET $Z=26$. I $=28$ PROJ $Z=2, N=0$ $G=4.230 \quad E=33,500$

SCALE FACTDR $=1.350$. FRAC PREEO $=0.043$

- P H RHOU/RHO STRU/STR STRD/STR

$\begin{array}{lllll}2 & 0 & 9.896 E-01 & 9.896 E-01 & 9.896 E-01 \\ 3 & 1 & 8.373 E-01 & 8.206 E-01 & \text { E.206E-01 } \\ 4 & 2 & 5.637 E-01 & 5.722 E-01 & 5.240 E-01 \\ 5 & 3 & 3.264 \mathrm{E}-01 & 3.079 E-01 & 2.333 E-01 \\ 6 & 4 & 1.509 E-01 & 1.353 E-01 & 6.615 E-02 \\ 7 & 5 & 5.744 E-02 & 5.872 E-02 & 1.043 E-02\end{array}$




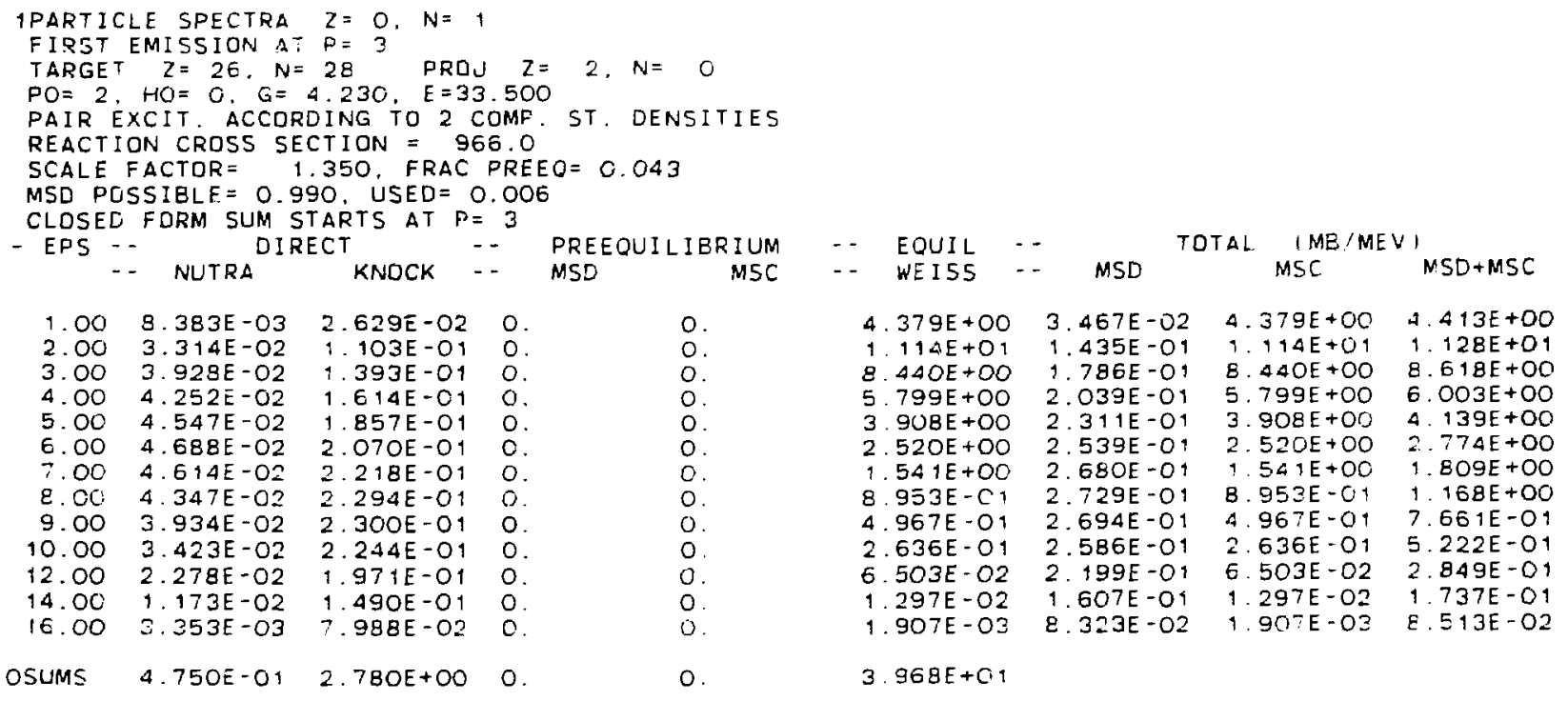




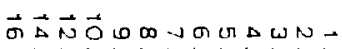
8888888888888 $\omega v \rightarrow N A v-N \omega \in v \omega$

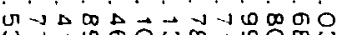

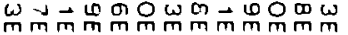
óóóóóóóóóó

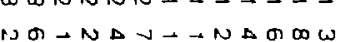
Jư

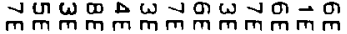

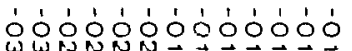
$N G \rightarrow N \Delta N \rightarrow+\infty \Delta \sigma \infty \omega$

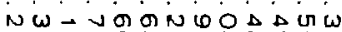

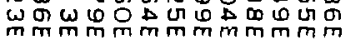

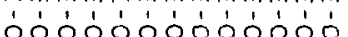

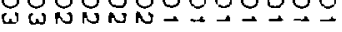

$\rightarrow \Delta-N \Delta \infty-N \omega \Delta+\omega \omega$ m- vo

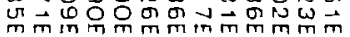

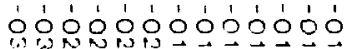

- + -

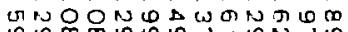

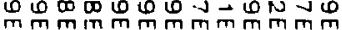
óóóóó WWNNNNA $\rightarrow-\infty$ U ๑O

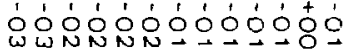
- $\omega \rightarrow \omega \sigma \rightarrow+N \Delta \sigma \infty \rightarrow+$ ค

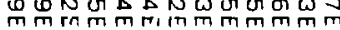

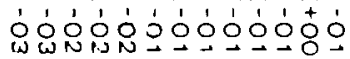
$\rightarrow \omega+\omega \sigma \rightarrow \rightarrow \Delta \sigma m \rightarrow \Delta$

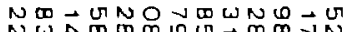
NWA óóóóóóóóóó

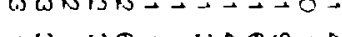
$\overrightarrow{0}$. w a 요

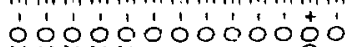

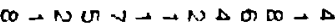

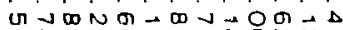

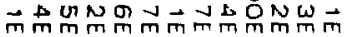
ó:0́

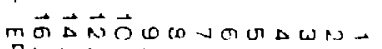
in 80800000080 $N \Delta \Pi \infty \rightarrow N \omega \Delta \sigma \omega \rightarrow \Delta$

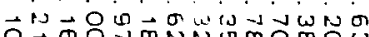
Ommmm óóóóóóóóó N $\triangle$ N

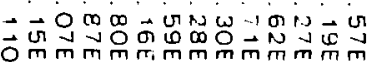
óóóóóóóóóț NW

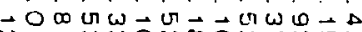
N $\overrightarrow{0}$ m

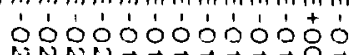
- WU - - NNAUM - . - va odoa on lo ó

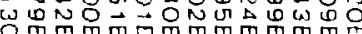

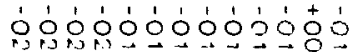

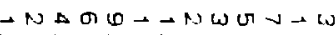

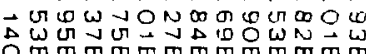

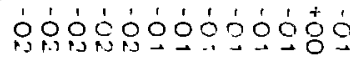

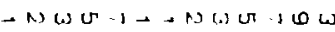

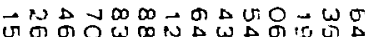

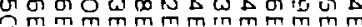

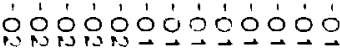
$\rightarrow+\omega \Delta \sigma \omega \rightarrow \cdots \omega \Delta \sigma \infty$

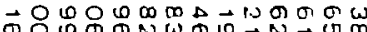

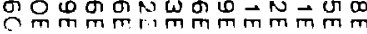

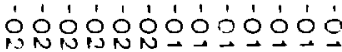

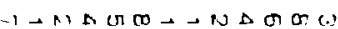
$\therefore$ us

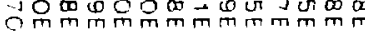

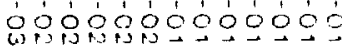

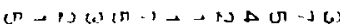

- $N$ N

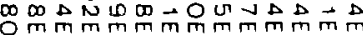
óćóóóóóóóó $n \omega+\omega \Delta+\ldots \omega w+1$. वं $-100 \sigma \sigma$
0

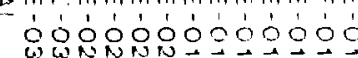

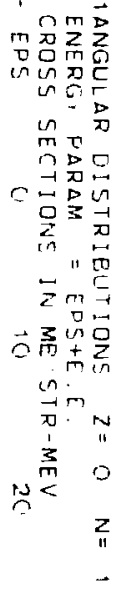

$\omega$

$\stackrel{5}{6}$ 


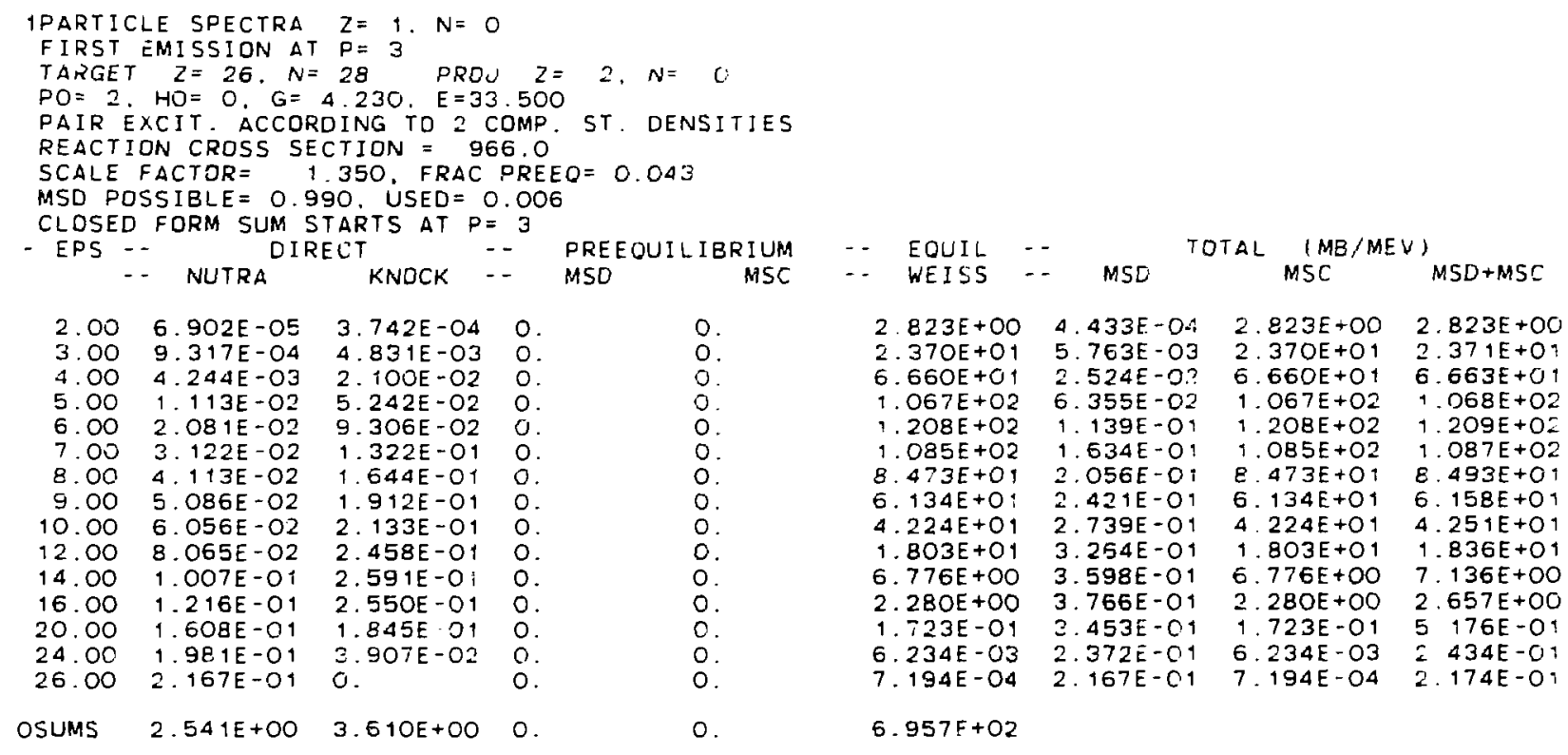




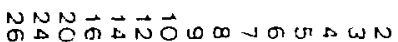
888888898888898

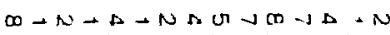
कó

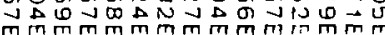

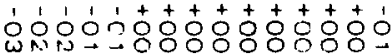
$\sigma \infty N \rightarrow \Delta-\omega \Delta \sigma \sqrt{\omega}+\Delta-N$ Ons

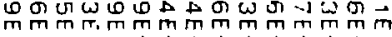

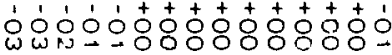

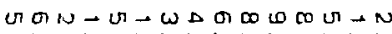
$N-1 \omega \infty N \omega N-10 \omega \omega N \rightarrow$ O

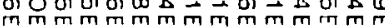

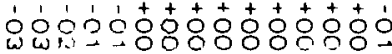
Derpin + (n)

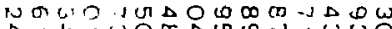

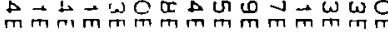

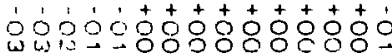

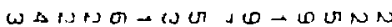
GmsNNG-1 B

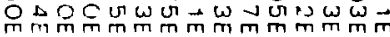

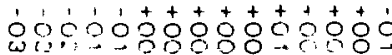

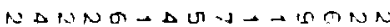
ตN ผ่

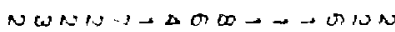

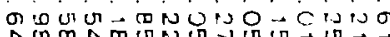

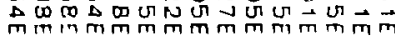
ód

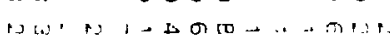
$\triangle$ का म中

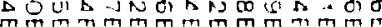

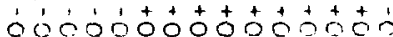

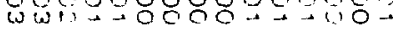
M G M N

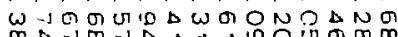

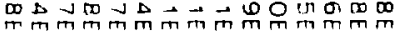
1 $11^{1} 1+t+t+t+t+1$

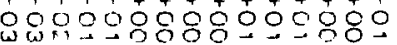
WNUN-A. $\triangle \sigma_{\infty}$ - A GNN $\rightarrow \infty$ on $\rightarrow$ on o o w

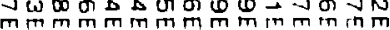

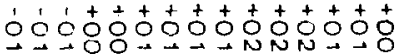

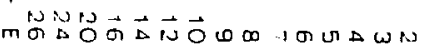
ingo8808888088080

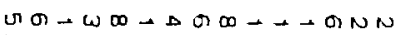

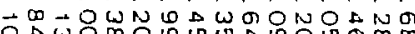
的mmmmmmm

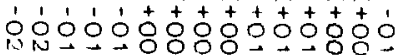

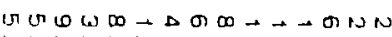

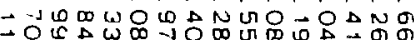

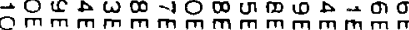
อ́

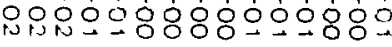
ת n $\rightarrow$ UUNATINONOBONNA Nom

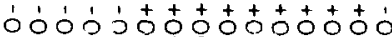
ming

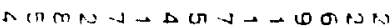

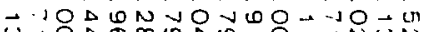

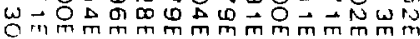

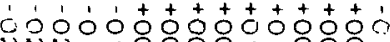

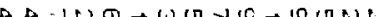

- gusana

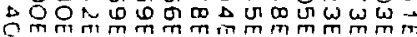

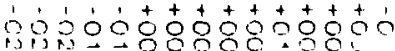

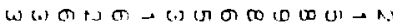

स्य

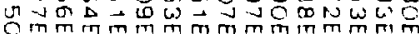

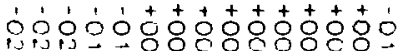

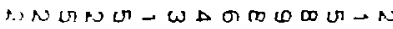
- 约

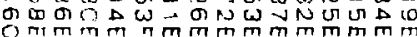

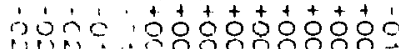

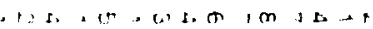

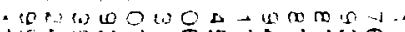

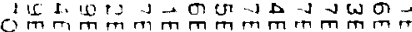

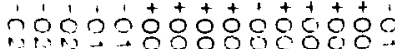

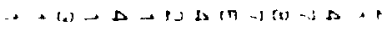

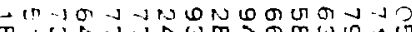

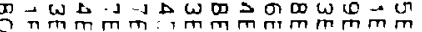
رि:

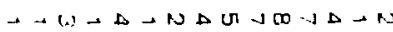
古 Dmmmmmmmmm mmm ‘

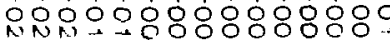

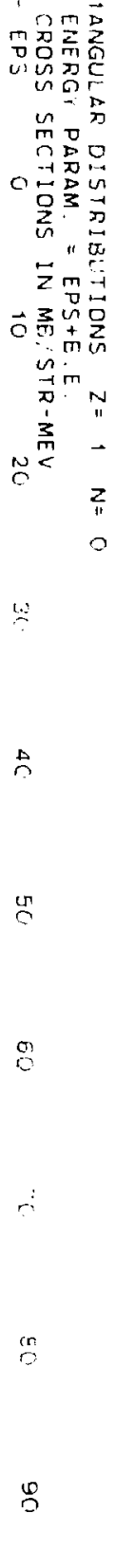




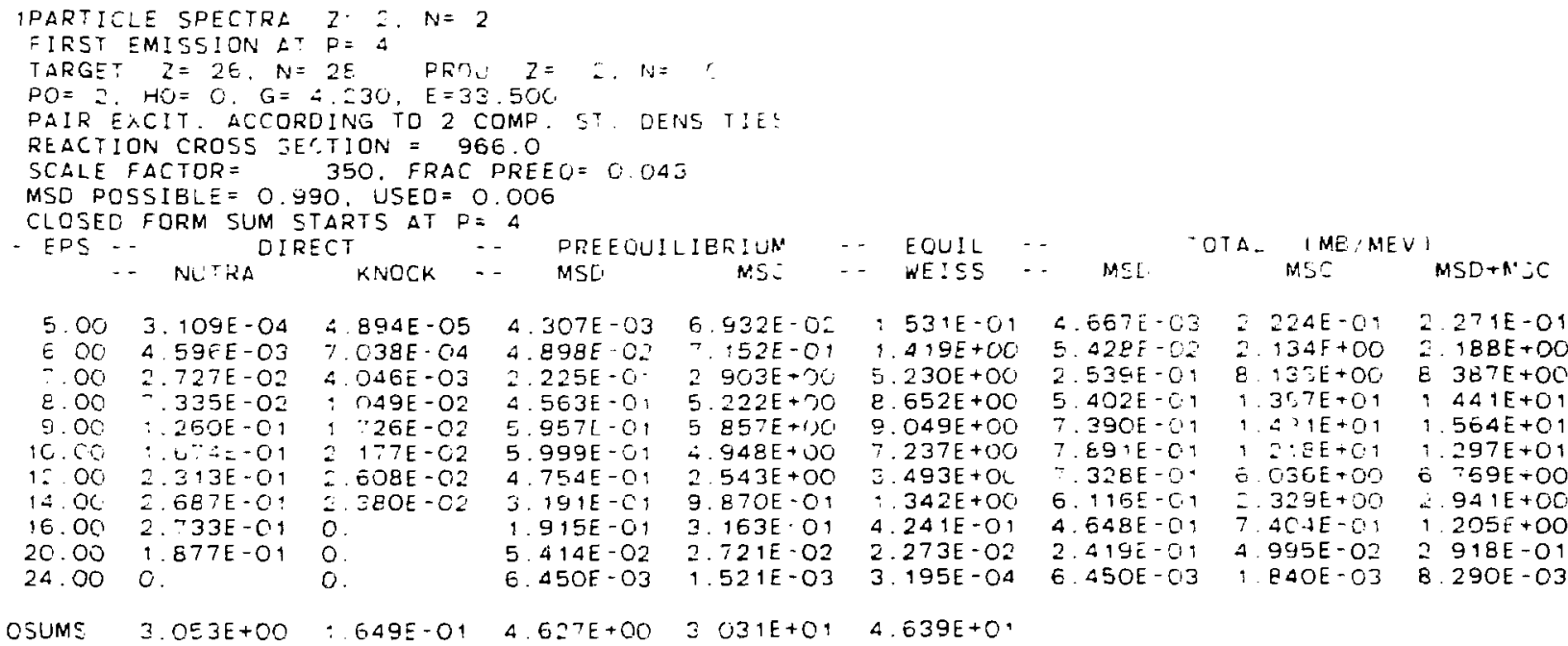

OSUME

$3.053 E+00: 649 E-01$

4. $627 E+00=031 E+01 \quad 4.639 E+01$ 
CROSS SECTIONS IN ME/STR-MEV

$\begin{array}{llll}\text { EPS } & 0 & 10 & 20\end{array}$

30

40

50

60

70

80

90

$\begin{array}{lllllllllllll}5.03 & 2.34 E-02 & 2.32 E-02 & 2.25 E-02 & 2.14 E-02 & 2.01 E-02 & 1.88 E-02 & 1.76 E-O 2 & 1.66 E-O 2 & 1.59 E-O 2 & 1.56 E-O 2\end{array}$

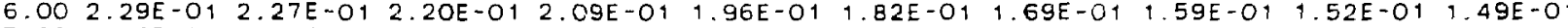

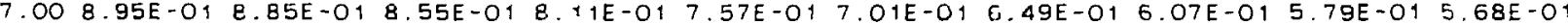

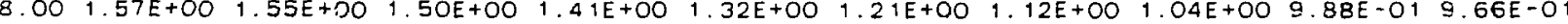
$9.001 .74 E+O D \quad 1.72 E+O O \quad 1.66 E+O O \quad 1.56 E+O O \quad 1.45 E+O O \quad 1.33 E+O O \quad 1.22 E+O O \quad 4.13 E+O O \quad 1.06 E+O O \quad 1.04 E+O O$

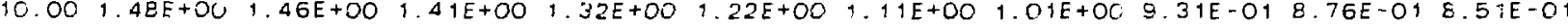
12.00 8.28E-01 8. $16 \mathrm{E}-01$ 7.81E-01 7.29E-01 6.65E-01 5.99E-01 5.38E-01 4.87E-014.51E-01 4.33E-01

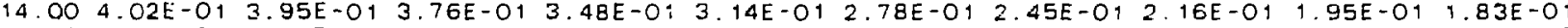

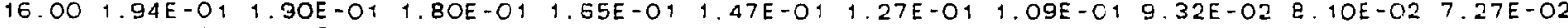
$20.006 .68 E-02 \quad 6.53 E-02 \quad 6.12 E-02 \quad 5.49 E-02 \quad 4.75 E-02 \quad 3.96 E-02 \quad 3.22 E-C 2 \quad 2.57 E-02 \quad 2.04 E-02 \quad 1.63 E-02$ $24.002 .06 E-03 \quad 2.00 E-03 \quad 1.86 E-03 \quad 1.65 E-03 \quad 1.4 C E-03 \quad 1.14 E-03 \quad 8.99 E-046.97 E-04 \quad 5.38 E-044.23 E-04$ $\begin{array}{lllllllllll}- & -1 P S & 100 & 110 & 120 & 130 & 140 & 150 & 160 & 170 & 180\end{array}$

$5.001 .58 \tilde{E}-0=1.64 E-02 \quad 1.73 E-O 2 \quad 1.8 A E-02 \quad 1.97 E-02 \quad 2.09 E-02 \quad 2.19 E-02=25 E-02 \quad 2.58 E-02 \quad 2.27 E-01$

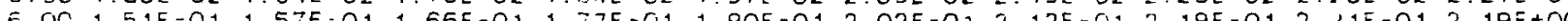

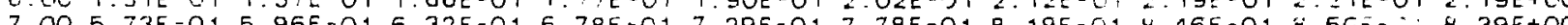

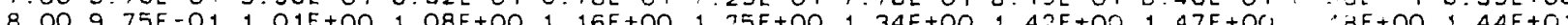

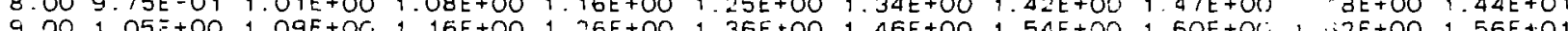

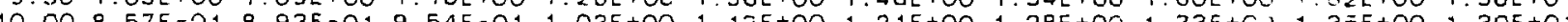
.

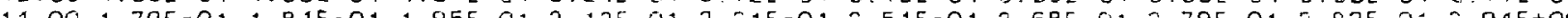

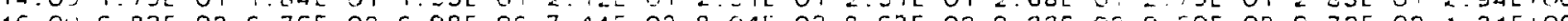

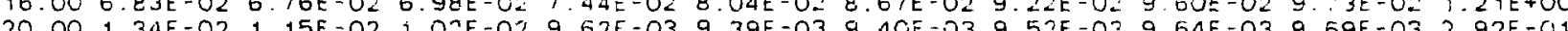
$24.003 .45 E-042.99 E-042.77 E-042.73 E-042.81 E-042.96 E-04$ 3. $11 E-04 \quad 3.22 E-043.26 E-04$ 2. $29 E-03$ 
$-5=10.28 \mathrm{MEV}$

- OCCUPATION PROEAEILITIES PO= 2. HO=

TARGET $Z=26, N=28$ PROU $Z=1, \mathrm{~N}=0$

$G=4.230, E=33.500$

SCALE FAC ${ }^{-} O K=1.350$. FRAC PREEO $=0.537$

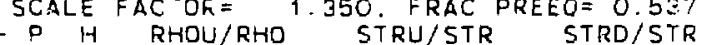

$\begin{array}{lllll}1 & 9 & 1.000 E+00 & 0 . & 0 . \\ 2 & 1 & 8.680 E-01 & B .680 E-01 & 8.680 E-01 \\ 3 & 2 & 6.725 E-01 & 6.594 E-01 & 5.956 E-01 \\ 4 & 3 & 4.489 E-01 & 4.075 E-01 & 3.082 E-01 \\ 5 & 4 & 2.498 E-01 & 2.030 E-01 & 1.112 E-01 \\ 6 & 5 & 1.201 E-01 & 9.209 E-02 & 2.593 E-02 \\ 7 & 6 & 4.876 E-02 & 4.55 E E-02 & 3.496 E-03\end{array}$


IPARTICLE SPECTRA $Z=0 . N=1$

FIRST EMISSION LT P = =

TARGET $Z=2 E . N=28$ PROU $Z=1 . N=0$

$P \cap=2, H O=1, G=4.230, E=33,500$

PAIREXCIT ACCOROING TO 2 COMP.

REACTION CROSS SECTION = 966.0

SCALE FACTOR= 1.350. FRAC PREEO=0.537

MSO POSSIBLE $=0.868$. USED $=0.405$

- CLOSED FORM SUM STARTS AT $P=2$.

- NUTRA

KNOCK -

PREEOUILIBRI JM

- EOUI -

MSD TOTAL IMB/MEII

MSD+MSC

$\begin{array}{rr}1.00 & 0 . \\ 2.00 & 0 . \\ 3.00 & 0 . \\ 4.00 & 0 . \\ 5.00 & 0 . \\ 6.00 & 0 . \\ 7.00 & 0 \\ 8.00 & 0 . \\ 9.00 & 0 . \\ 10.00 & 0 . \\ 12.00 & 0 . \\ 14.00 & 0 . \\ 16.00 & 0 .\end{array}$

osuins

0 .

1. $134 E+00$

$\begin{array}{rrr}78 E-01 & 2.3 B 1 E+O C & 1.134 E+00 \\ 756 E+00 & 6.259 E+O 0 & 4.321 E+O O\end{array}$

. $.993 E+O 0 \quad 1.527 E+O 0$

. $335 E+O O-1.24 O E+O C$

$.591 E+O C \quad 4.993 E+00$

$155 \mathrm{E}+00 \quad 5.335 \mathrm{E}+00$

$2.999 E+00 \quad 4 \cdot 133 E+00$

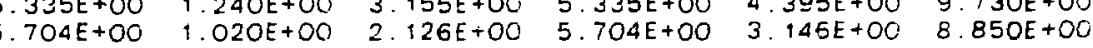

$958 \mathrm{E}+00 \quad 8.293 \mathrm{E}-0 \quad 1.371 \mathrm{E}+0 \mathrm{O} \quad \mathrm{5} .958 \mathrm{E}+00 \quad 2.201 E+00 \quad 8.159 E+O O$

$\begin{array}{llllll}.030 E+00 & 6.594 E-01 & 8.384 E-01 & E .03 O E+O 0 & 1.498 E+00 & 7.528 E+00\end{array}$

E. $93 B E+O D \quad 5.138 E-01 \quad 2.873 E-01 \quad 5.93 B E+O D \quad 1 . O C 1 E+O O \quad 6.939 E+O O$

$5.722 E+00$ 3.935E-01 2.704E-01 5.722E+00 6.639E-01 $6.385 E+O 0$

$5.415 E+00 \quad 2.967 E-01 \quad 1.435 E-01 \quad 5.415 E+O 0 \quad 4.402 E-01 \quad 5 . B 55 E+O O$

$4.637 E+O O \quad 1.613 E-01 \quad 3.541 E-O 2 \quad 4.637 E \div O O \quad 1.968 E-01 \quad 4 . B 33 E+O D$

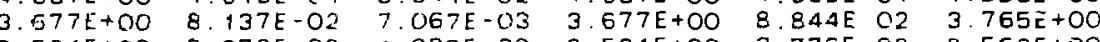

$2.524 \mathrm{E}+00 \quad 3.672 \mathrm{E}-02 \quad 1.039 \mathrm{E}-03 \quad 2.524 \mathrm{E}+00 \quad 3.776 \mathrm{E}-\mathrm{C2} \quad 2.562 \mathrm{E}+00$

7. $746 E+C \cdot 9.598 E+O C 2158 E+O:$ 
1ANGULAR DISTRIEUTIONS $Z=0 \quad N=1$
ENERGY PARAM = EPS+E. E

- EPS O 10

30

40

50

60

$7 \mathrm{C}$

80

90

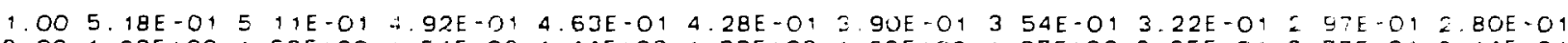

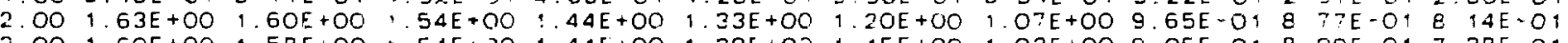

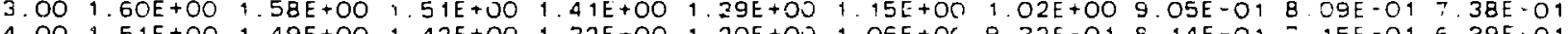

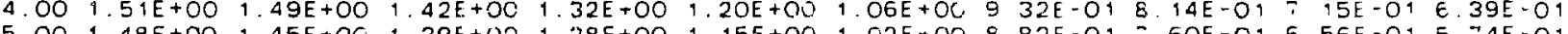

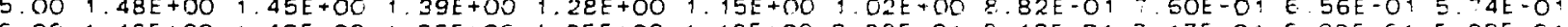

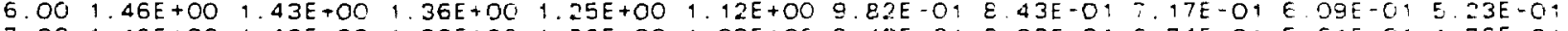

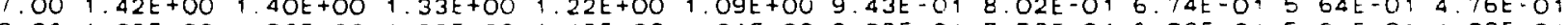
$8.001 .38 E+001.36 E+001.28 E+00118 E+001.04 E+00$ E. $188 E-01 \% .58 E-016.30 E-015215-012.33 E-01$

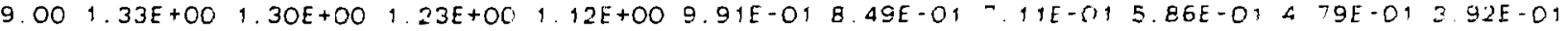

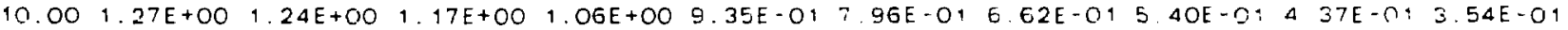

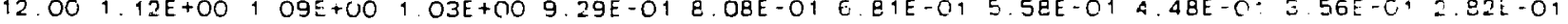

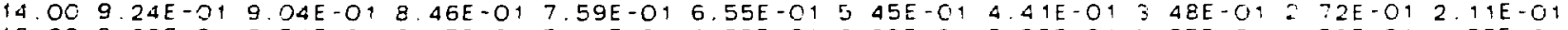

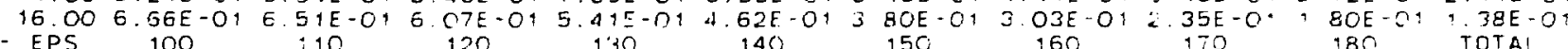

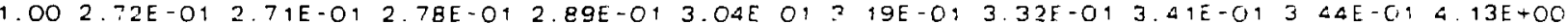

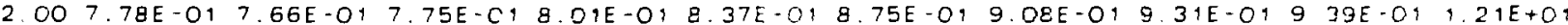

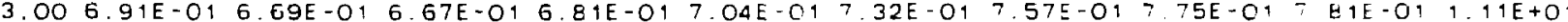
$4.005 .86 E-015.55 E-015.43 E-015.45 E-015.57 E-015$ T4E-O1 5.90E-01 6.01E-01 $5.06 E-019.73 E+00$

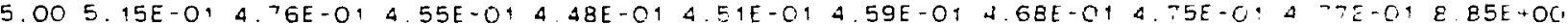

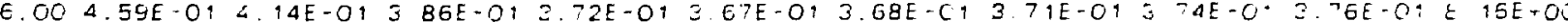

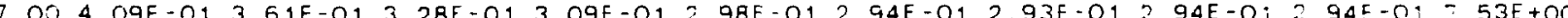
$3 E-012.33 E-01231 E-01231 E-01254 E+00$ $76 E-012.80 E-012.5 B$

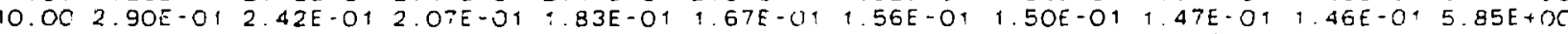

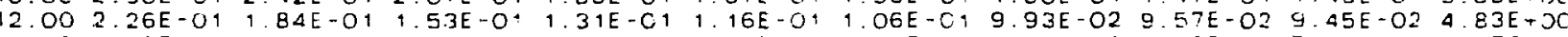

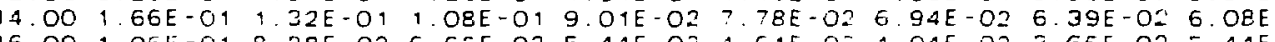

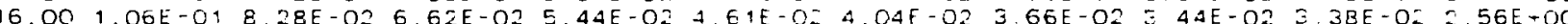


IPARTICLE SPECTRA $Z=1 . N=0$

FIRST EMISSION AT P $=2$

TARGET $Z=26 . N=28$

PRUL $7=1, N=C$

$P O=2, H O=1, G=4.230, E=33.500$

PAIR EXCIT ACCORDING TO 2 COMP ST DENSITIES

REACTION CROSS SECTION = 966.0

SCALE FACTOR $=1.350$. FRAC PREEO $=0.537$

MSD POSSIBLE $=0.868$, USED $=0.405$

CLOSED FORM SUM STARTS N NUTRA DIRECT

PREEOUTLIBKIUM

KNOCK .. MSO

MSC

- EQUIL MSO TOTAL (MB/MEV)

$\begin{array}{rr}2.00 & 0 . \\ 3.00 & 0 \\ 4.00 & 0 . \\ 5.00 & 0 . \\ 6.00 & 0 . \\ 7.00 & 0 . \\ 8.00 & 0 . \\ 9.00 & 0 . \\ 10.00 & 0 . \\ 12.00 & 0 . \\ 14.00 & 0 . \\ 16.00 & 0 . \\ 20.00 & 0 . \\ 24.00 & 0 . \\ 26.00 & 0 .\end{array}$

26.000

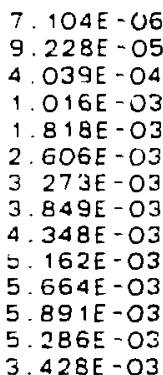

7. $37 \mathrm{GE}-02$

8. $329 E-01$. $237 E-01$

3. $191 \mathrm{E}+00 \quad 7.890 E+O 0$

WEISS

MSO

MS

$M S D+M S C$

$9.034 E-02 \quad 2.940 E+02 \quad 1.086 E+02 \quad 3.783 E+O 2$

1. $327 E+O$

1. $534 E+O O$

1. $83 E E+00 \quad 1.911 E+00$

5. $201 E+01$ T. T91E+DO $4.409 E+0: \ldots .728 E+01$

$5.801 E+017.074 E+007.128 E+01 \quad \div .836 E+01$

$1.124 E+01-1.582 E+0$

6. $565 E+01$

$124 \mathrm{E}+\mathrm{O}$

B. $148 E+0,9.272 E+01$

$.630 E+01,261 E+0$

. $901 E+01$

1. $441 E+O 1$

$409 E+01$

E. $E 5 O E+01$

$1.739 E+019.917 E+O O$

$335 E+01$

1. $739 E+01$

$5.867 \mathrm{E}+21$ T. $498 \mathrm{E}+0$

$1.794 E+0 i 7.566 E+O C$

2. $297 E+01$

$4.327 E+01$ G. $066 E+01$

1.813E+O1 $4.282 E+00$

9. $210 E+00$

$1.794 E+01 \quad 3.054 E+01 \quad 4.848 E+01$

3. $687 \mathrm{E}+0 \mathrm{O}$

$\begin{array}{lll}813 E+01 & 1.409 E+01 & 3.222 E+01 \\ 736 E+01 & 6.098 E+O O & 2 \\ 345 E+O 1\end{array}$

1. $227 E+01 \quad 4.382 E-01 \quad 9.379 E-02$

6. O98E+0O

$345 E+01$
$875 E+01$

$\begin{array}{lllll}3.428 E-03 & 7.248 E+00 & 1 & 163 E-01 & 3.397 E-03 \\ 0 . & 4.263 E+00 & 5.051 E-02 & 3.921 E-04\end{array}$

5. $320 E-01,281 E+01$

1. $197 \mathrm{E}-01 \div .372 \mathrm{E} \cdot \mathrm{rOC}$

$4,263 E+\infty 0$ 5. $091 \mathrm{E}-02 \quad 4.314 \mathrm{E}+00$ 


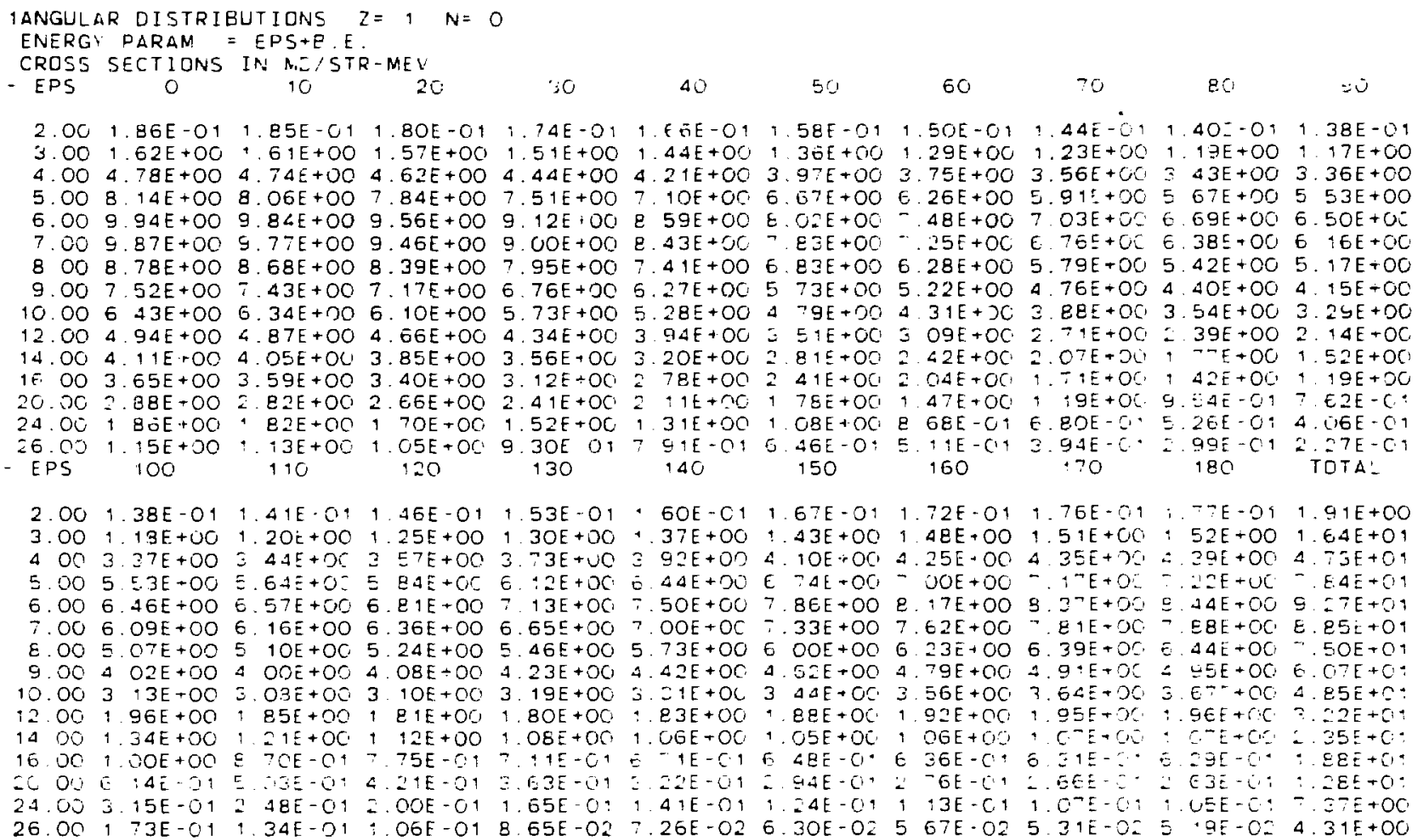


IPARTICLE SPECTRA $Z=2, N=2$

FIRST EMISSION AT $P=4$

TARGET $I=26 . N=2 \varepsilon$ FRO $Z=\therefore N=O$

$P Q=2 . H O=1, G=4=30 . E=33.500$

PAIR EXCIT. ACCORDING TO 2 COMP. S: DENDITIES

REACTION CROSS SECTION = 966.0

SCALE FACTOR $=1350$. FRAC PREEO $=0.537$

MSD POSSIBLE $=0.86 \varepsilon . \quad$ USED $=0.405$

CLOSED FORM SUM STAETS AT $\mu=4$
EPS - - NUTRA OIREC
KNDCK - M MSE
MSE OUILIERIUN

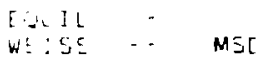
TOTA (ME/MEVI
MSE MSD+MSE
$\begin{array}{ll}5.00 & 2.009 E-03 \\ 6.0 C \quad 2.809 E-02\end{array}$
507E-05
4. $508 E-04$
was
$7.00 \quad 1.872 E-01$
E $\triangle 33 E-03$
2. $383 \mathrm{E}-03$
8. $793 E-0$
$224 E+O=$
$254 E E-03 \cong 103 E-0: 9.357 E-02$
$800 \quad 3.972 E-01,706 E-02$
4. $920 E-O=$
$3071 E-C T=E 12 E+00$
$42 \mathrm{E}-02$ \& $4 \mathrm{E} 3 \mathrm{E}-0 \mathrm{O}$
E $797 \mathrm{E}-07$
9.0O E $385 E-01=883 E-02$
$\begin{array}{lll}10.00 & 7.899 \mathrm{E}-0: & 2.759 \mathrm{E}-02 \\ 12.00 & 9.302 \mathrm{E}-0 . & 1.946 \mathrm{E}-02\end{array}$
14.00 8.933E-0. 5. $345 E-02$
16.00 7.1B3E-0, 4.825E-O2
$20.00 \quad 2.321 E-01$
24.000
$490 E-C 2 \quad 5.208 E-01 \div 859 E+00$
4 E35E-O. $5159 E+O 0 \quad 5 \quad 522 E+O O$

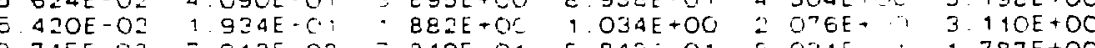
3. $745 E-O 2-9+2 E-O Z$ - $240 E-O 1$ \& $842 E-O 1$ E O31E $1787 F+O O$

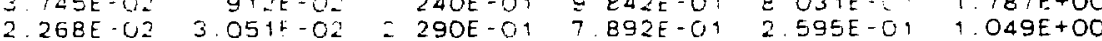
2.26BE-C2 3.051H-O2
$372 \mathrm{E} \cdot \mathrm{O}, \mathrm{C}$
2. $321 E-01$ O.
0 .
0
2
$73 \mathrm{IE}-\mathrm{OA}$
$737 E-O 4$ - $1.737 E-O A$
OSUMS $\cong .13 E E+00 \quad 4.605 E-015146 E-01 \quad 2.689 E+00 \div 4 G-0+01$ 


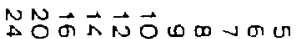
88898898888

$\omega-v-\rightarrow \omega A \omega \pi n$

$\rightarrow \rightarrow D$ D $\rightarrow$ N $\rightarrow$ L

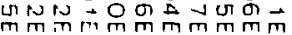

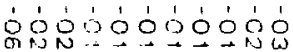
$\rightarrow \omega \& \omega+\omega \& \omega \mapsto \sigma \sigma$ O-

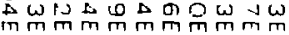

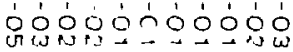
$\rightarrow W \Delta L-\omega E D N \sigma_{1}$ is $\theta \Delta \Delta, 0 O \omega \rightarrow \Delta O \vec{A}$

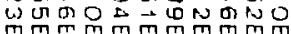
b́óó

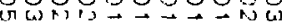

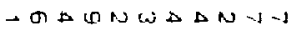

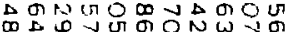

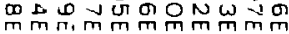

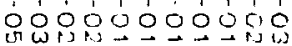

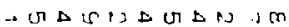

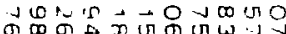

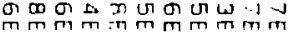
óódodóón

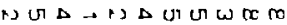
ovwOUNAOOOU

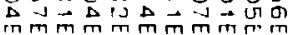
óód่óódóó NUS. A

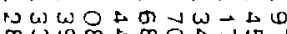

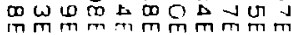

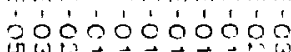

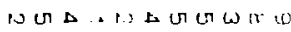

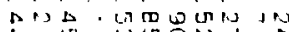

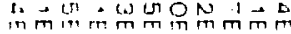

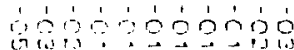
, In

b. A . U

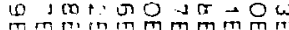

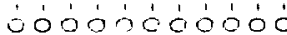
0 .

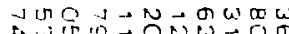

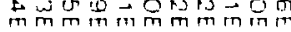

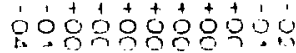

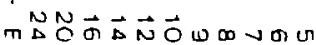
ing88808808?

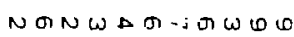

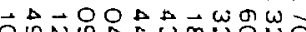

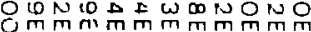

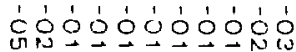

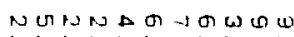

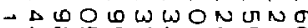

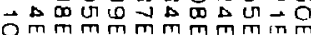
ด́óó0́óó

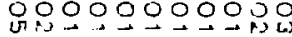

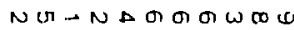
$\rightarrow N O \omega D-O O O \Delta \omega \omega$

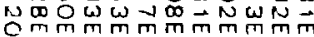
óóóćcóóóó

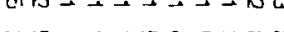

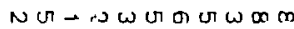

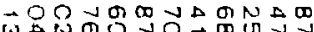

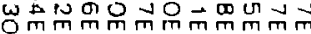
óㅇoㅇóóóó . . .

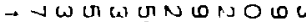

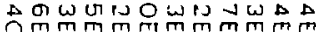

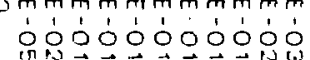
$\rightarrow \omega \rightarrow \mathrm{\omega} \Delta$ × $\rightarrow$ on wo- va co no $\omega$

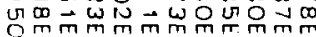
óćóóóódóó $\rightarrow N \rightarrow+N \Delta \Delta \Delta N \sigma]$

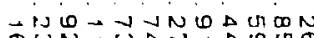
gîn ớóóósóóóó + w w w wa wa $\rightarrow O \omega-\Delta$ o i $\rightarrow \Delta \Delta \infty$

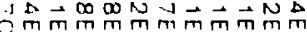
gígcogógó

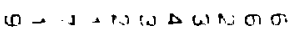

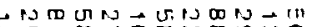

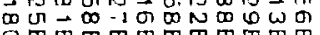
- o

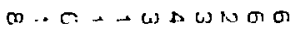
Önn

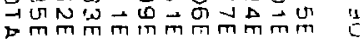
pób́丶万ó

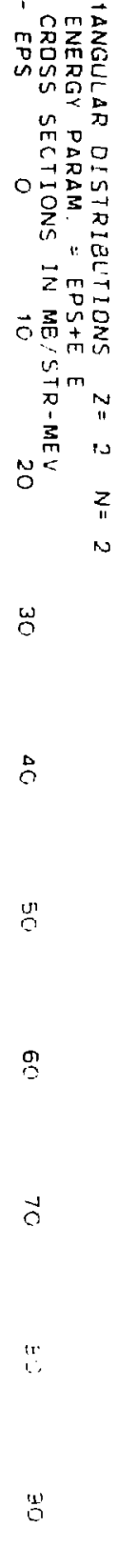


$-5=10.28 \mathrm{MEV}$

- OCCUFATION PROEAEILITIES PO= 2. MC: = .

TARGET $Z=2 E . N=2 E$ DROJ $Z=1 . N=0$

$G=4.231 . E=66.000$

SCALE FACTOR= +350, FRAC PREEQ $=0.916$

$P$ H RHOU,RHO STRU/STR STRD/STR

$10 \quad 1.000 E+00$

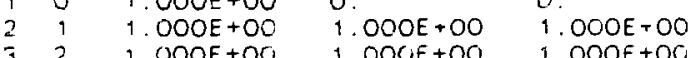

$32,0 O O E+O O \quad 1.000 E+O O \quad 1.0 O O E+O O$

$43 \quad 1.0 O O E+O O \quad: .0 O O E+O O \quad 1.00 O E+O 0$

$549.581 E-01 \quad 9.387 E-01 \quad 9.387 E-0$

$655.680 E-01 \quad 8.558 E-01 \quad 8.151 E-O 1$

$76-7.513 E-01 \quad 7.499 E-C 1 \quad 6.399 E-O$

$\begin{array}{lllll}8 & 7 & 6.175 \mathrm{E}-01 & 6.154 \mathrm{E}-01 & 4.353 \mathrm{E}-01 \\ \text { y } & 8 & 4.794 \mathrm{E}-01 & 4.751 \mathrm{E}-01 & 2.442 \mathrm{E}-\mathrm{O}\end{array}$ 


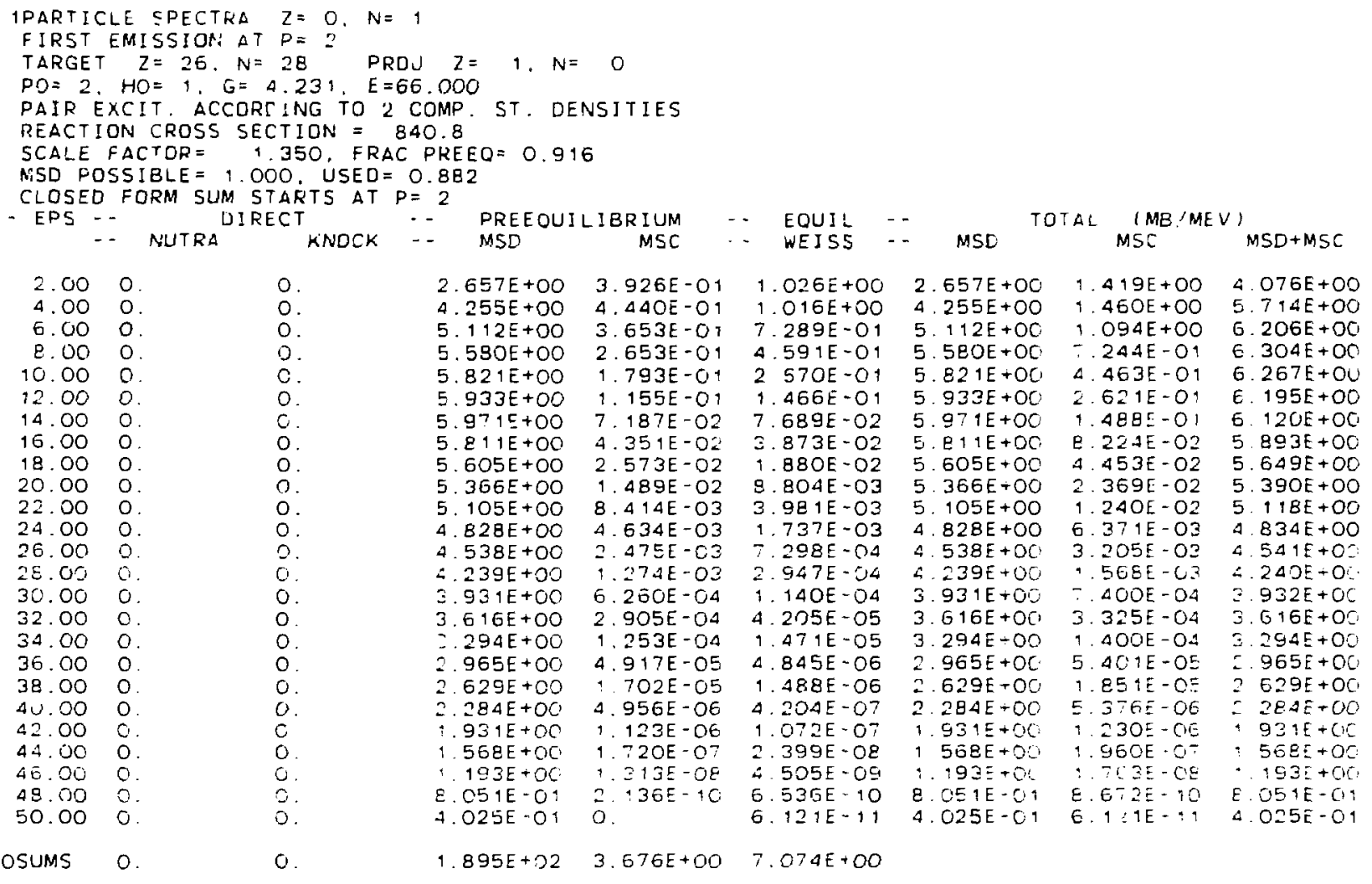


$2.006 .42 E-015.32 E-015.05 E-015.64 E-015 . \$ 3 E-014.58 E-01 \quad 4.03 E-013.52 E-013.09 E-012.73 E-01$ $4.009 .83 E-019.67 E-019.23 E-01$ B. $94 E-017.7 O E-016.79 E-015.88 E-015.05 E-014.34 E-013.75 E-01$

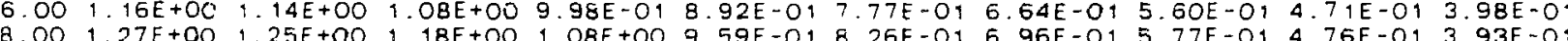
$10.00,36 E+001.33 E+001.26 E+001.14 E+001.00 E+008.54 E-017$ OSE-O $15.79 E-014.5 B E-013.79 E-01$ $12.00,43 E+O 0,4 O E+O O, 32 E+O O, 19 E+O C, O 4 E+O 0 B 72 E-017,1 E E-015,74 E-014.56 E-013.62 E-01$

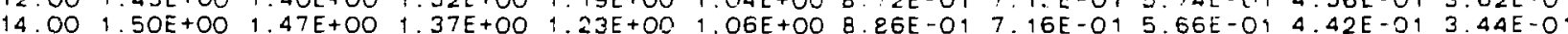

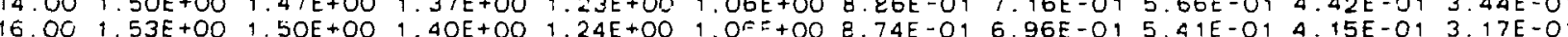

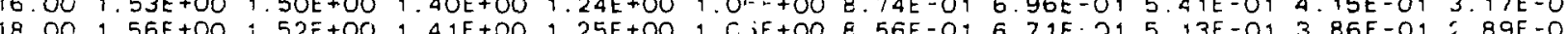

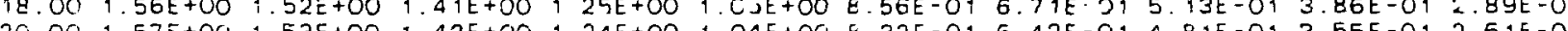
$22.00 ; .59 E+00 \quad 1.54 E+00 \quad 1.42 E+O 0 \quad 1.23 E+00 \quad 1.02 E+00 \quad 8.03 E-01 \quad 6.08 E-014.47 E-013.23 E-0: 2.32 E-0$ $24.001 .59 E+001.54 E+00 \quad 1.41 E+001.22 E+00$ 9.92E-01 $7.69 E-015.71 E-014.11 E-012.90 E-012.05 E-01$

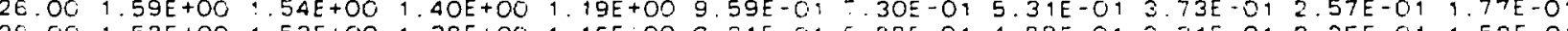

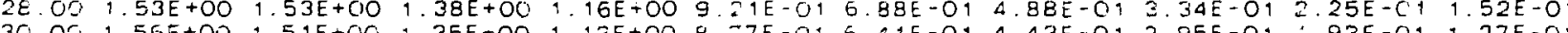

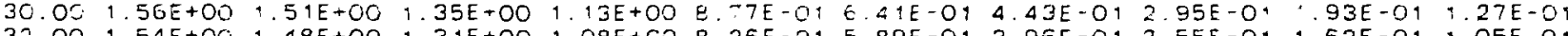

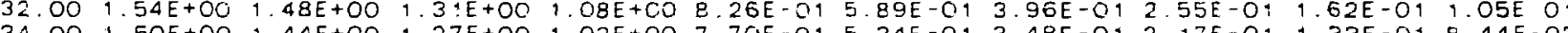

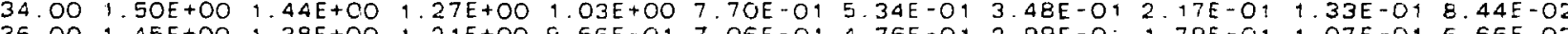
$36.001 .45 E+00$ 1. $38 E+001.21 E+00$ 9.66E-C1 $7.06 E-014.76 E-012.99 E-0.1 .79 E-01 \quad 1.07 E-016.66 E-02$ $32.001 .38 E+001.32 E+00 \quad 1.14 E+C 0$ B. $93 E-01 \quad 6.36 E-014.14 E-012.49 E-01 \quad 1.43 E-018.25 E-025.13 E-02$

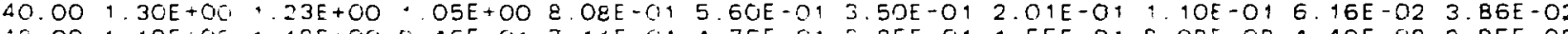

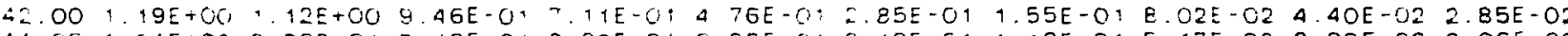

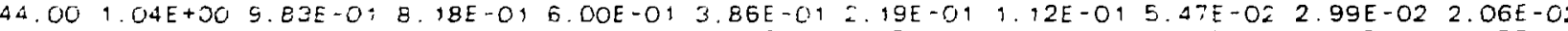

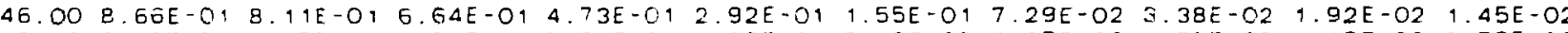
$48.006 .39 E-01 \quad 5.95 E-014.78 E-013.30 E-01 \quad 1.93 E-01 \quad 9.49 E-02 \quad 4.05 E-02 \quad 1.79 E-02 \quad 1.13 E-O 2 \quad 9.53 E-03$

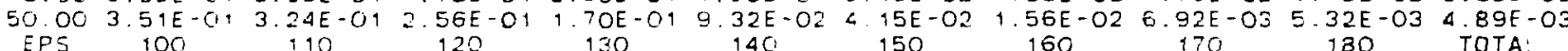

$\therefore O O=47 E-0: 2.29 E-012.19 E-012.14 E-01213 E-01=14 E-012.17 E-012.19 E-012.20 E-014.08 E+O 0$

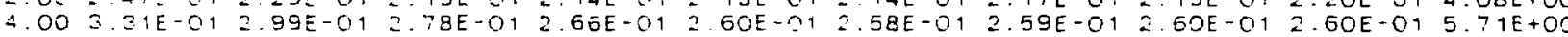

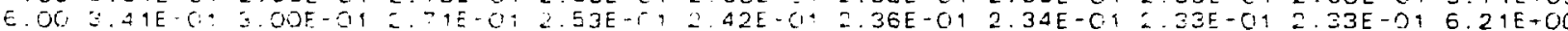
E.00 3.29E-D: 2.81E-01 2.47E-O1 2.24E-012.09E-01 2.0OE-01 1.95E-01 $1.93 E-019.92 E-016.30 E+00$

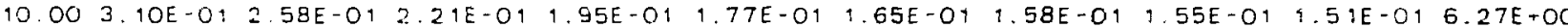
$12.002 .89 E-012.36 E-01 \quad 1.96 E-014.69 E-01 \quad 1.49 E-01: 36 E-01 \quad 1.28 E-01 \quad 1.24 E-01 \quad 1.22 E-016.20 E+00$ $14.002 .69 E-012.15 E-01 ; .75 E-01,1.47 E-011.27 E-011.13 E-011.04 E-019.94 E-02 \quad 5.7 B E-026.12 E+00$ $15.00244 t-01,90 E-011.52 E-011.25 E-01,06 E-019.24 E-02 \quad 8 \quad 36 E-027.87 E-027.71 E-025.89 E+00$ $18.00218 E-01167 E-01,31 E-01,06 E-01879 E-027.53 E-02$ G $69 E-026.21 E-C 2 \quad 6.05 E-02 \quad 5.65 E+00$

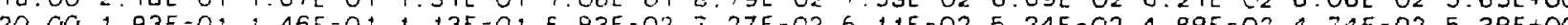

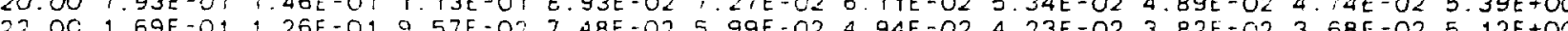
24 .

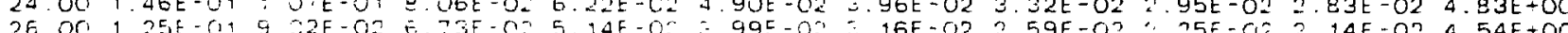

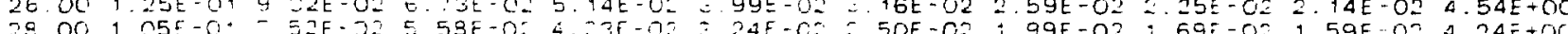
200 .

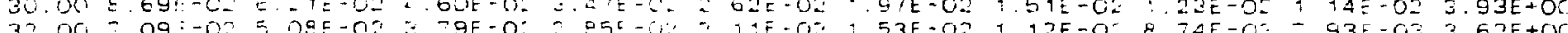

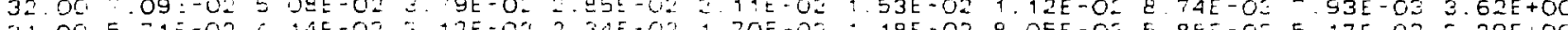
34.00 E $30.004 .54 E-3.36 E-02=58 E-1.93 E-02$ 1.

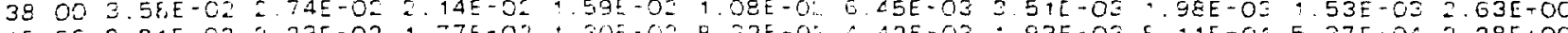

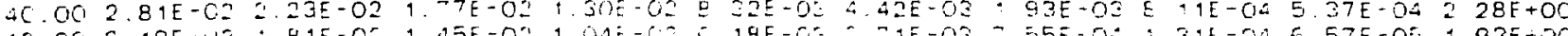

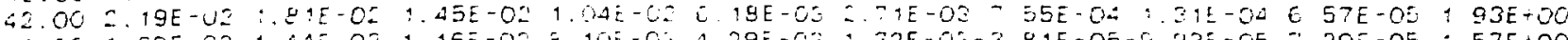

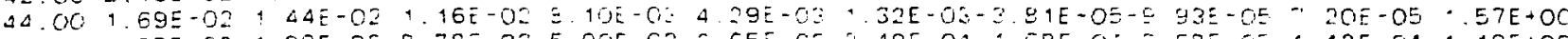

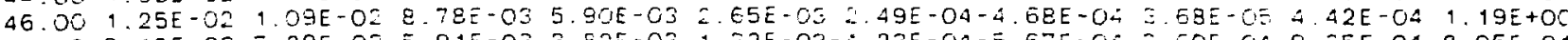
$42.008 .42 E-C 3-.29 E-035.91 E-033.82 E-03 \quad 1.32 E-03-4.23 E-04-5 . E 7 E-043.60 E-04025 E-042.05 E-01$ $50.004 .19 E-033.57 E-032.95 E-03$ 1.86E-0? 3.97E-04-5.75E-04-3.89E-04 5.30E-04. $04 \mathrm{E}-034.02 \mathrm{E}-01$ 
IPARTICLE SPECTRA $Z=1, i N=0$

TARGET $Z=26 . N=28$ RROU $Z=1 . N=0$

$P O=2, H O=, G=4.231, E=66.000$ ST. DENSITIES

REACTION CROSS SECTION =

SCALE FACTOR= 1.350. FRAC PREEQ $=0.916$

$M S D$ POSSIBLE $=1.000$, USED $=0.882$

CLOSED FORM SUM STARTS AT $P=2$

CLOSED FORM SUM STARTS AT PE

$$
\begin{array}{llll}
\text { D DIRECT } & \text { FREEQUILIBRIUM } \\
\text { - NUTRA } & \text { KIVGCK } & \text { P } & \text { MSE MSC }
\end{array}
$$

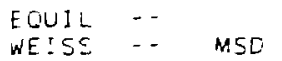
TOTAL (NE:MEV)

.957E-09 8.526E-05

8. $199 E-05$

8. $526 E-05$
2. $754 E+00$

3. $661 E-O$
9. $473 E-O$

. $22 E-04$

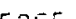

$M 50+M 50$

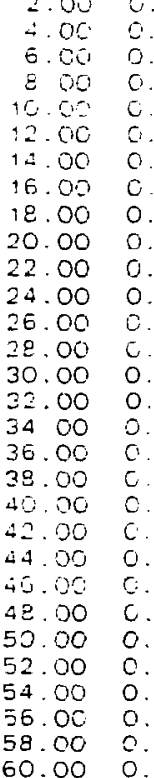

osums
3. $685 E-04$

ว. $313 E-04$

$7.657 E-04$ 1. $143 E+01$

9. $713 \mathrm{E}-\mathrm{O} 4$

1. $146 \mathrm{E}-03$

1. $293 \mathrm{E}-03$

1. $414 E-03$

1. $508 E-03$

. $579 E-03$

1. $652 E-03$

$1.657 \mathrm{E}-03$

$1.544 \mathrm{E}-03$

1. $613 \mathrm{E}-03$

$.565 E-03$

1. $427 \mathrm{E}-03$

1. $3395-03$

i 2395-03

1. $130 E-03$

$055 E-04$

$7935 E-04$

6. $713 E-O 4$

5. $338 E-04$

5. $388 E-04$

0 .

6. 055E-02

1. $\triangle E 3 F_{2}+01$

1. $993 E+00$

$4.220 E+\infty O$

1. $894 E+00$

2. $438 E+00$

2. $764 \mathrm{E}+\mathrm{O}$

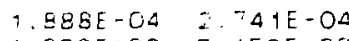

$262 E-O C$

6.

$151 E+O C$

4. $386 E+00$

$7.906 E+00$

$150 E_{-}+00$

$4 E 2 E+D C$

$.288 E+00 \quad 1 \quad 193 \mathrm{E}+\mathrm{C}$

E. $550 E+00 \quad 1.98 E+O$

$1.490 E+O 1 \quad 1.012 E+O$

$364 E+0$

E. $69 E E+O C$

$=.034 \mathrm{E}+\mathrm{O}$

$874 E+O 0$

पS $3 E+0$

$1494 E+O$

. $570 E+00$

$920 E \div 0 ?$

. $203 E-01$

$5.802 E-01$

$.475 E+0$

$\therefore-2 E T E+D O$

$7 E 5 E+C 1$

$1.421 E+01$ 1.548E-O

3.038E-01

1. $449 E+O$

E67E+C:

1. $372 E+01$ 9. $102 E-02$

$537 E-O:$
$527 E-C j 2$

1. $372 E+O$

$618 \mathrm{E}-0$

$301 E+01$ 5.258E-O?

$3.574 E-02$

$301 E+C$

$-01 \quad 1.452 E+O$

$.227 E+04$ 2. $995 E-02$

i. E45E-02

$227 E+C$

$4629 \mathrm{E}-\mathrm{O}=2.32 \mathrm{~F}+\mathrm{O}$

$152 E+01$
$1.662 E-O 2$

. $33, E-03,152 E+C$

$395 E-02$ i. $154 E+0 i$

3. $162 E-03 \quad 1.076 E+01 \quad 1.222 \vec{E}-02 \div .077 E+0$

$994 \mathrm{~F}+00$ 4. $804 \mathrm{E}-03$

$228 E+00 \quad 2.467 E-03$

$317 E-03 \quad 9.596 E+00$

6. $22 \mathrm{E}-03 \div \mathrm{OOOE}+0$.

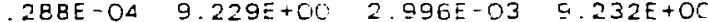

2. O3EE-O4 \&. $462 E+00 \quad 1 \quad 420 E-03 \quad 8.464 E+00$

$7.515 E-05 \quad 7.696 E+00$ E. $44 E E-04 \quad J . E 97 E+0 C$

$2.637 E-O E$ E. $933 E+O E \quad 2.765 \bar{E}-04$ E. $932 E+00$

$.695 E+00 \quad 5.697 E-04$

$6.172 E+00 \quad 1,011 E-04$

$5.416 E+00$ 3 $6 E 7=-05$

$4.758 E+00$ I. $171 E-05$

4. $107 E+00$ 3. $005 E-06$

4. $107 E+00$ 3. $305 E-06$

$\begin{array}{ll}3.432 E+O O & 5.902 E-O 7 \\ 2.729 E+O O & 6.854 E-08\end{array}$

$\begin{array}{llll}1.819 E-09 & 1.993 E+00 & 4.797 E-09 & 1.293 E+00 \\ 2.219 E-10 & 1.220 E+00 & 2.219 E-10 & 1.20 E+00\end{array}$

1. $099 E-04$ D $173 E+00$

TAE-OG D. $173 E+C$

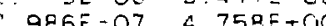

3. $939 E-05$

5 . $17 E+O$

$4.758 E+0 C$ 1.251E-05 $4.58 E+00$

$4.108 E+00$ 3. $290 E-06 \quad 4.08 E+00$

$3.433 E+O 0$ 3. $230 E-06$ $4.4 D E+D O$

$2.730 E+00 \quad 7.932 E-O B \quad 2.73 O E+O D$

$1.730 E+00 \quad 7.932 E-O B \quad 2.73 O E+O D$

4. $797 E-09 \quad 1.993 E+00$

$=134 E+02 \quad 2.247 E-0, \quad 5.542 E+01$

. O39E-OT 


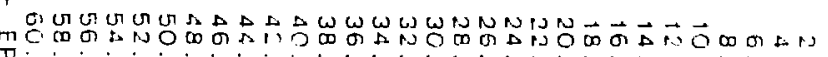

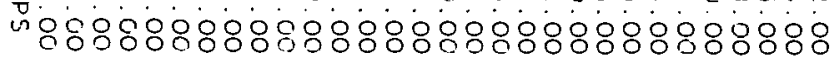

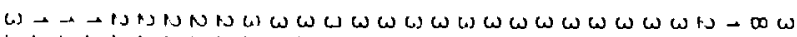
$\overrightarrow{0} 0$ o ○ी

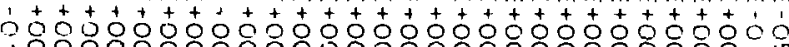
$-00000000000000000000008000-4$

6. - . ARNNWt

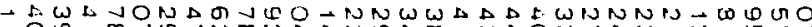

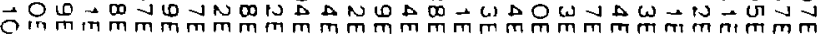

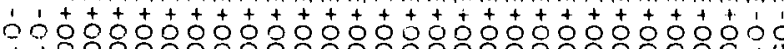
I

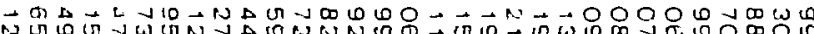
M

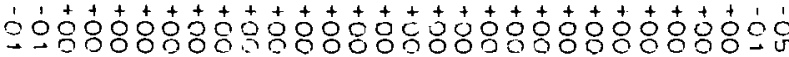
+ ש

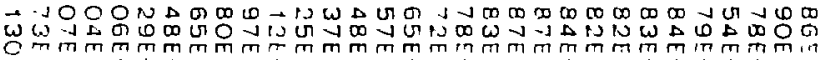

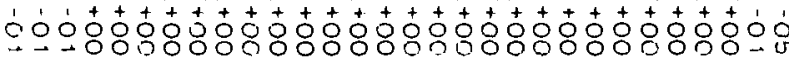

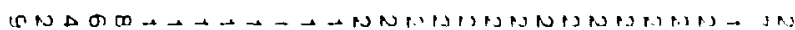

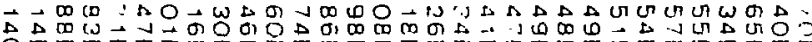

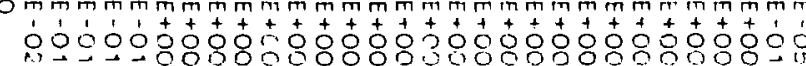
(.) N

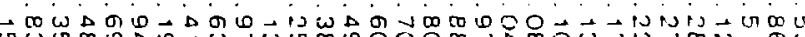
ज小

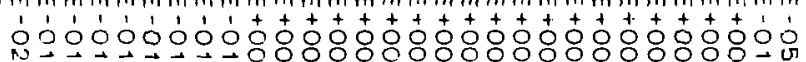

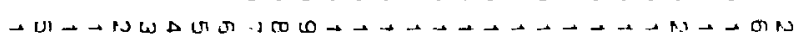

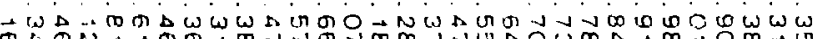

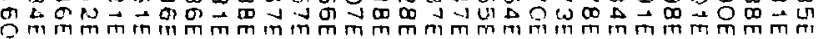

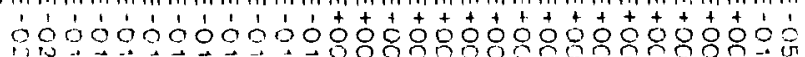

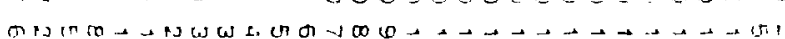

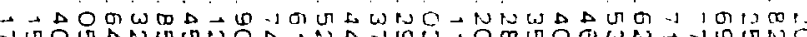

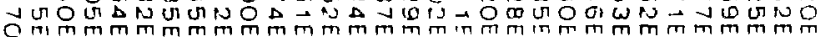

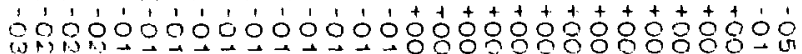

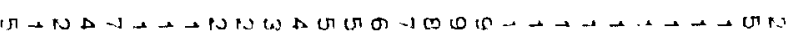
$\rightarrow \omega \sigma \omega \rightarrow 0 \omega \omega \omega$ o

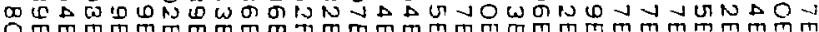
6.

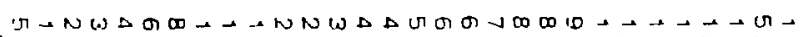

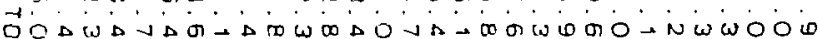

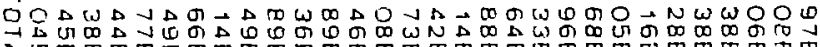

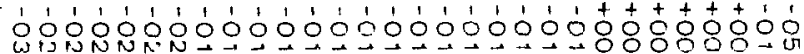




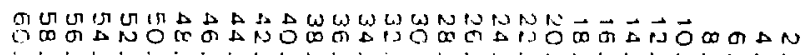
888888889888882888888898888888 מ A M N $\vec{\sigma}$ 小

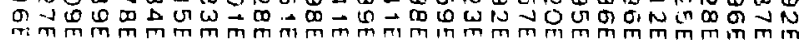

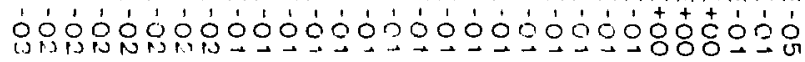

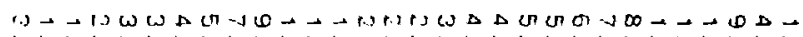

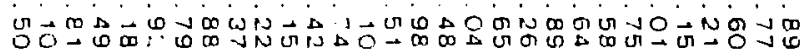

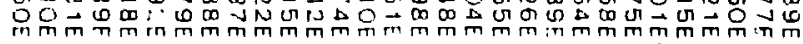

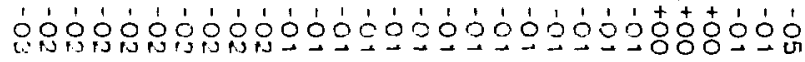

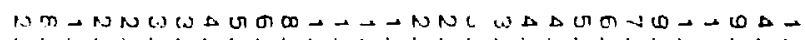
ம

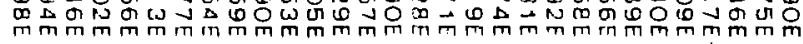

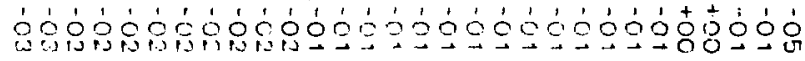

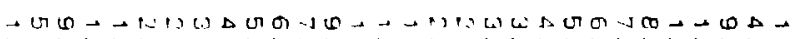

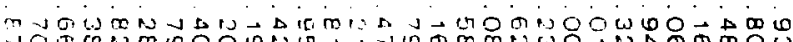

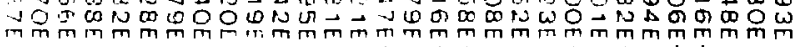
bo

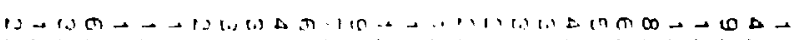

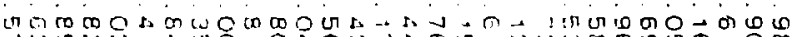
ㄴ.

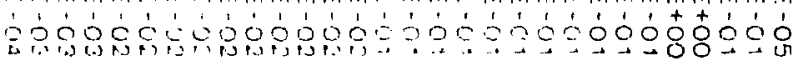
j.

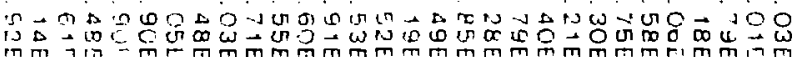

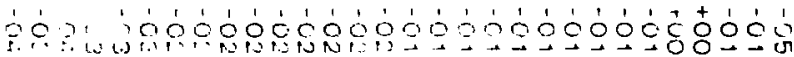

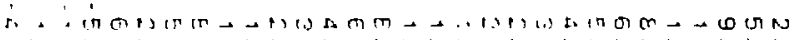

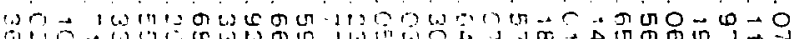

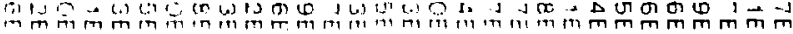

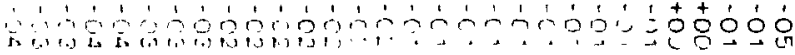
$1,0,1+0$

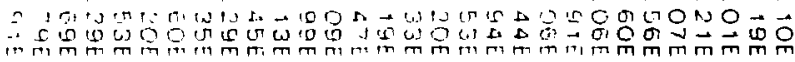

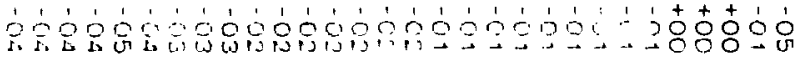

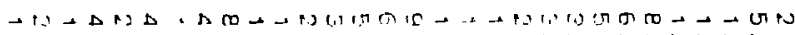

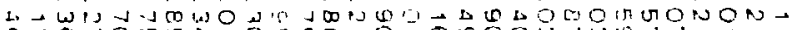

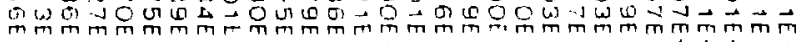

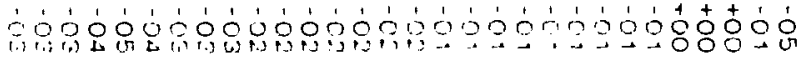

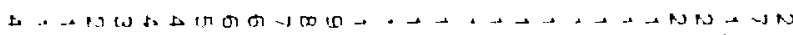
Б:

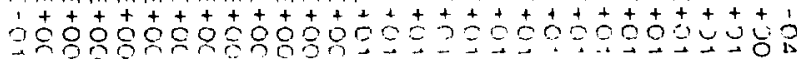


IPARTICLE SPECTRE $z=2$, iv- 2

FIRST EMISSION AT $P=4$

TARGET $Z=26 . N=28$ PROL $Z=1, N=$ =

$P O=2 . H O=1, G=4.221, E=66.000$

paIR FXCIT aCCDRDING TD 2 COMF. ST. DENS:TIES

REACTION CROSS SECTION = BAOR

SCALE FACTOR $=1.350$. FRAC PREEQ $=0.916$

MSD POSSIELE $=1.000$, USED $=0.8 \% 2$

CLOSED FORM SUM STARTS AT

EDS - .

NUTRA

KNOCK

MSO

MSC

- EQUIL -

$m 5$

TOTA! IME ' MEU U

$\begin{array}{lll}6.00 & 3.860 E-03 & 2.125 E-04\end{array}$

9. $239 \mathrm{E}-03$

$.035 E-02$

8. $395 E-02$

1. $331 \mathrm{E}-02$

$M S C$

MSD+MSO

10.00 8.499E-02 5.057E-O2 $9.170 \mathrm{E}-02$ - $.127 \mathrm{E}-02$

$12.001 .453 E-01 \quad 9.007 E-03 \quad 1.154 E-01 \quad 9.639 E-02$

$16.002 .284 \mathrm{E}-0 \mathrm{~T} 1.546 \mathrm{E}-02 \quad \mathrm{3} .7 \mathrm{AE}-02 \quad 5.525 \mathrm{E}-02$

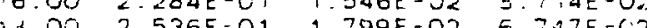

3.0O 2.536E-O $1.799 E-02$ G. TATE-C2

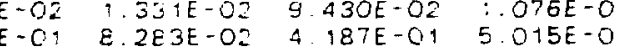

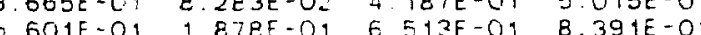

5 .

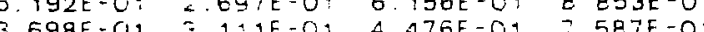

3.

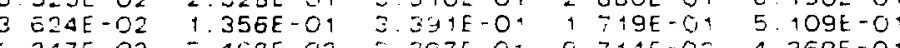

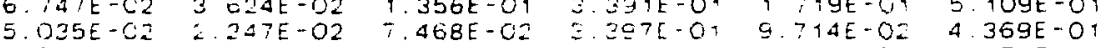
3.67OE-O2 $1.335 \mathrm{E}-02$ 3.926E-O2 $3.351 \mathrm{E}-0,015.261 \mathrm{E}-02$ 3.877E-O $\begin{array}{lllllll}2.634 E-02 & 7 & 672 E-03 & 1.982 E-O 2 & 3.26 O E-O 1 & 2.749 E-02 & 3.534 E-O\end{array}$ $1.873 E-02$

$299 \mathrm{E}-03 \quad 9.634 \mathrm{E}-03$

3. .29E-DI 1.393E-O2 3.2E8E-O1

$24.0022 .767 E-012.171 E-($

24.00 2.767E-O1 $2.293 E-02$

$2 \mathrm{C} .00$ 2.588E-01 2.49E-O2 1.323E-O2

$30.002 .42 B E-012.416 E-02 \quad 9.3 C 9 E-03$

$32.00 \quad 2.233 E-012.380 E-02$ ¿. $230 E-03$

$34.00 \quad 2.012 \mathrm{E}-\mathrm{O}$

2. $363 \mathrm{E}-03$

$4.515 E-03$

1. $279 E-03$

2. $04 \mathrm{OE}-\mathrm{O3}$

4. $566 E-03$

3. $5912 E-04$

3. $709 \mathrm{E}-0.4$

2.309E-O2 $4.566 E-03$

1. $858 E-04$

$6.877 E-03$

3. $030 E-0$

2. $205 E-03$

9. $39 \mathrm{TE}-\mathrm{OS}$

.

-. $31 \mathrm{BE}-03$

2. $736 E-01$

$38.00 \quad 1.524 E-01$

$891 E-02$

. $515 E-03$

$5.679 E-05$

. $388 \mathrm{E}-0$

$301 E-04$

$\therefore$ TSE-O

$\begin{array}{ll}40.00 & 1.273 E-0 \\ 42.00 & 1.028 \mathrm{E}-0\end{array}$

.68BE J2 1 O23E-03

$157 \mathrm{t}-05$

2. O6OE-US

. O25E-O

3. $344 E-04$

6. $830 E-04$

195E-O2 A. 39OE-O4

$4 \mathrm{E} .0 \mathrm{O}$ 5.807E-O2

ล. $.90 E-O 6$

$2.250 E-O 5$

$295 \mathrm{E}-0$

.

1. $207 \mathrm{E}-\mathrm{O}$

50 . 30 . $31 E$ E -52 5 ONOE-O3

52 OC $1123 \mathrm{E}-02$

$54.00 \quad 3.302 E-03$

56.000

$4-5 E-04$
$745 E-05$

$331 E-0$

4. $125 \mathrm{E}-\mathrm{OQ}$

$3 E-02$

(1) $5 E-02$

1. EASE-C2

2. $362 E=0.3$

1 $207 \mathrm{E}-0$.

I $4 E-C S \quad 9 \quad 47 \mathrm{JE}-02$

C. $452 E-067$ OASE-O?

e. $1295-09 ; .30 E-02$ E $425 \mathrm{E}-O E \div 130 \mathrm{E}-02$ .

o.

$.243 E-09$
$232 E-10$

$\begin{array}{lll}3.321 E-J 3 & 1.243 E-09 & 3.321 E-03 \\ 0 & 1.23 E-10 & 1.232 E-10\end{array}$

OSI):13

7. $\triangle 66 E+O O$

T. $31+E-01$

$1418 E+00$

Q. $445 E-01$ 


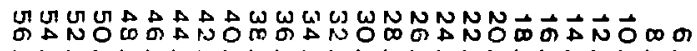
88888888888888888898888888

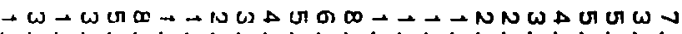

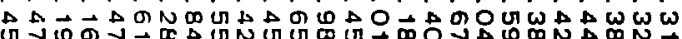

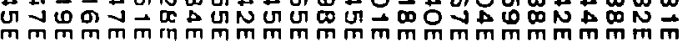

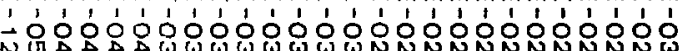

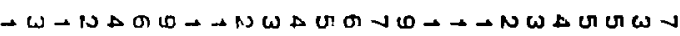

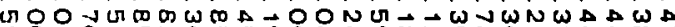

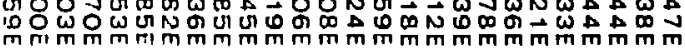

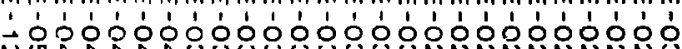
MNON UN U - - N N

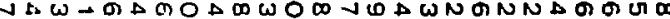

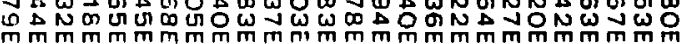

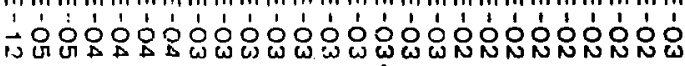

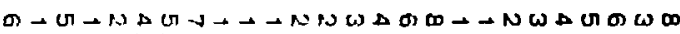

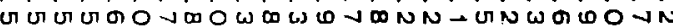

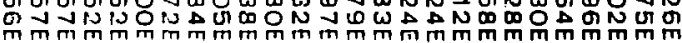

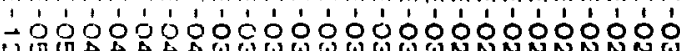

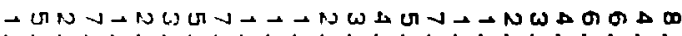

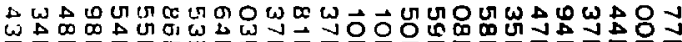

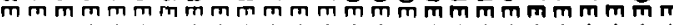

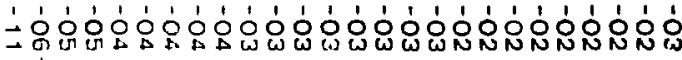

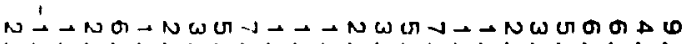
U $\omega \omega$ a

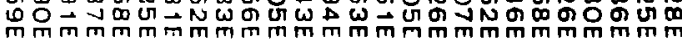

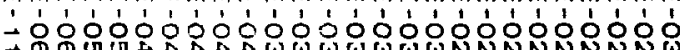
- Gดต Wi

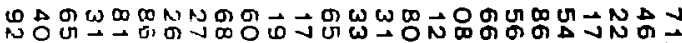
m mm m mm m m m m m m m m m m m m m m

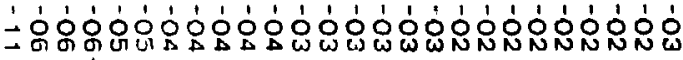

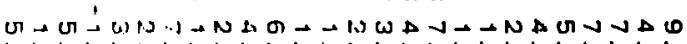

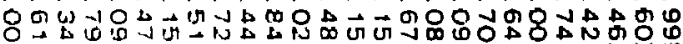

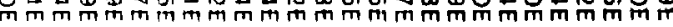
'

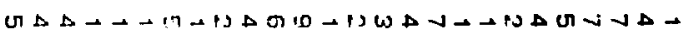

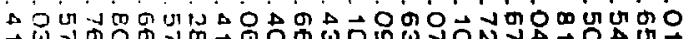
m

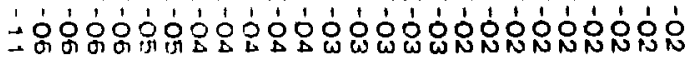

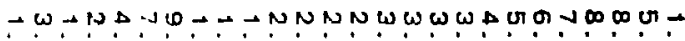

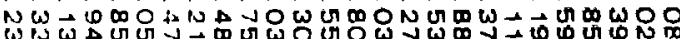

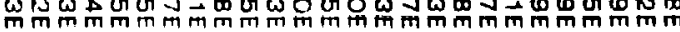

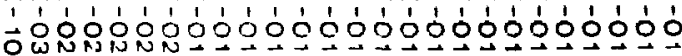

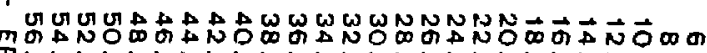

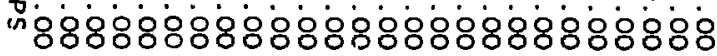

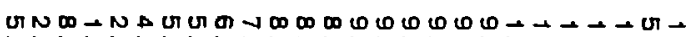

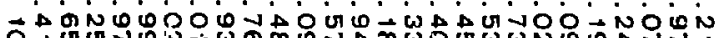

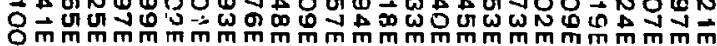

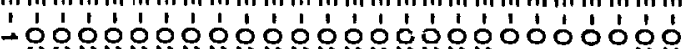

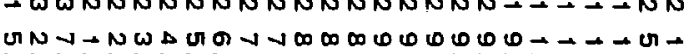

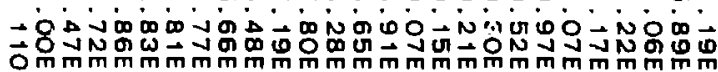

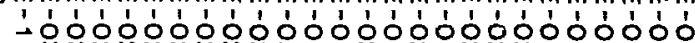
W-G-NWA $\vec{N}$

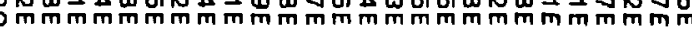

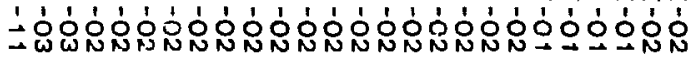

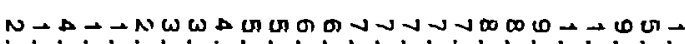

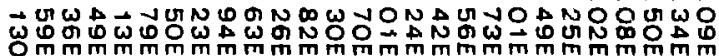

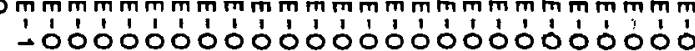
- OOWONO

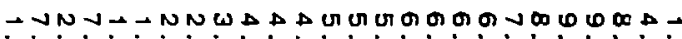

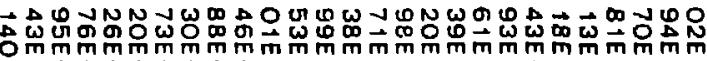

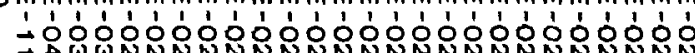

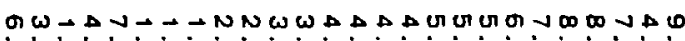

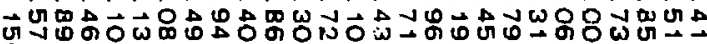

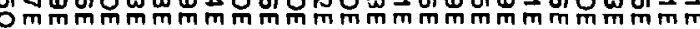

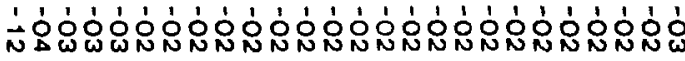

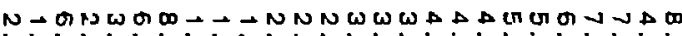

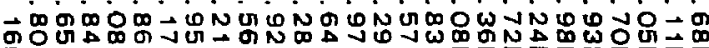

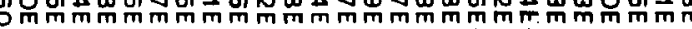

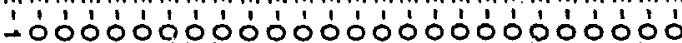

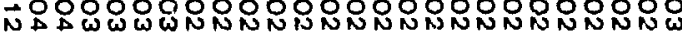

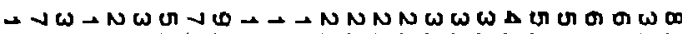

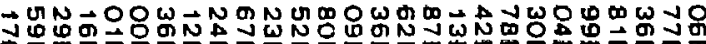
O

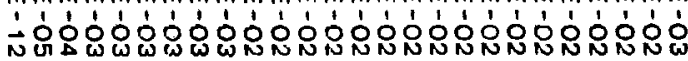

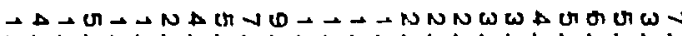

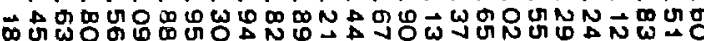

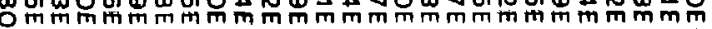

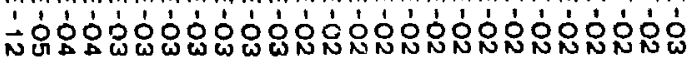
$\rightarrow \omega \rightarrow \omega N \rightarrow-N \omega$ G Gir w w

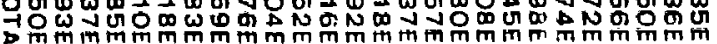

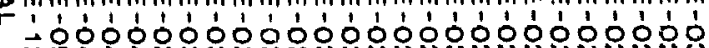

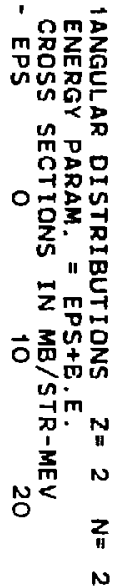

$\mathbf{\omega}$

\&

ษั

8

ò

\section{$\stackrel{\infty}{\mathbf{0}}$} NULD 
离

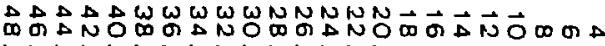
88888888888888888888880

N

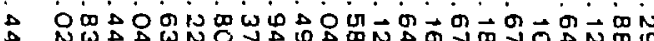

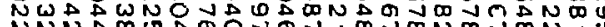
minmmmmm ơóóóóóóóóóóóóóóóóód

- 00000000000000000000000

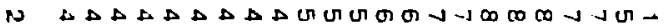
-

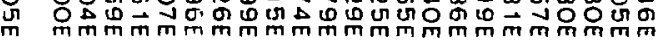
óóóóóóóóóóóóóóóó

0

N

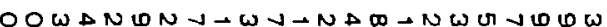

vN $A$ o 0 $\Delta 0$ O

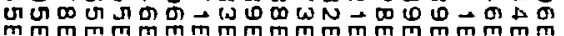

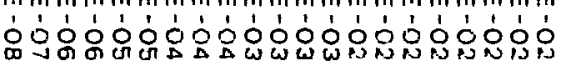

$N$

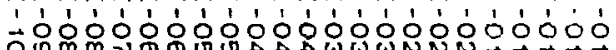

$J \omega \rightarrow \Delta-\Delta-\omega v \rightarrow \Delta \omega-\omega-\omega \omega \omega-$

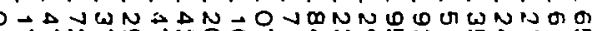

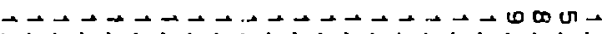
$\therefore$ w N

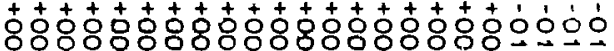

NNGUN-W

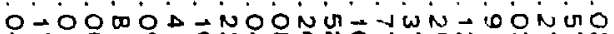
B $N$ G.

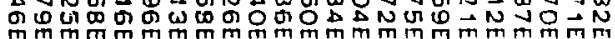

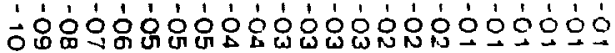

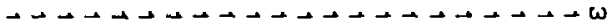

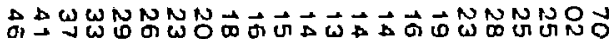

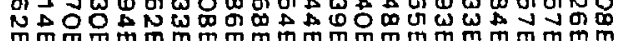

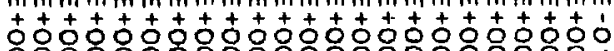

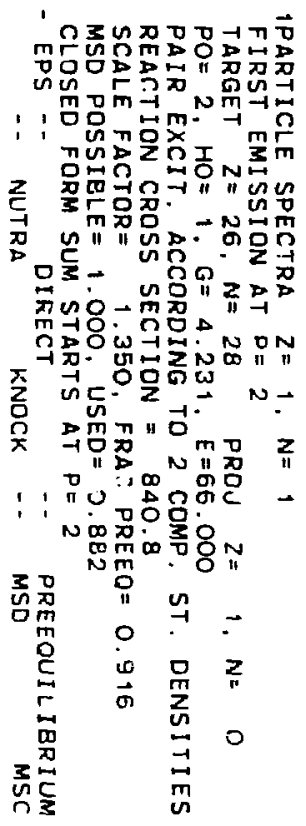

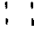

恶是

$\vdots:$

蛋

멈

帒主 


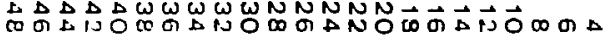
88888888888888888888888

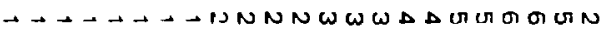
U

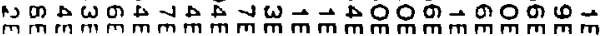

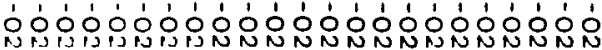
- + - + + + + - + NNNNWW

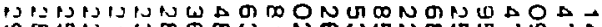
向

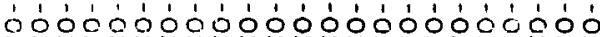

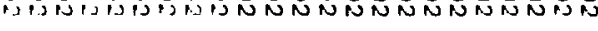

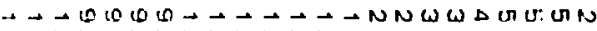
○ㅇำ

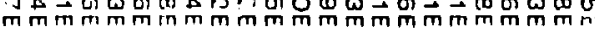

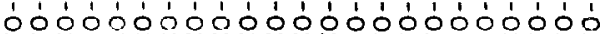
Th H त

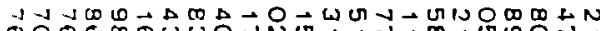
0)

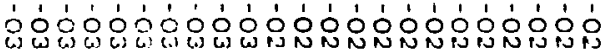

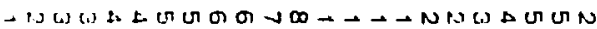
w. m m

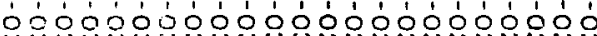
(1) i

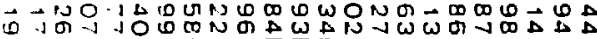
m m m m m m m m m m m m m m m m m m

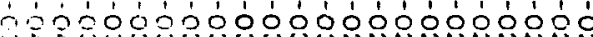

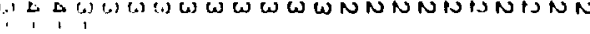
-

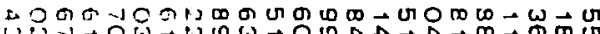
W

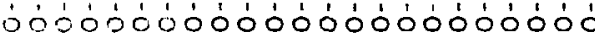
G. ดी 1.

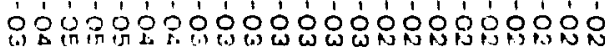

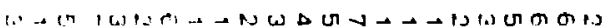

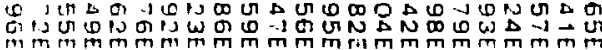

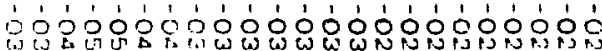

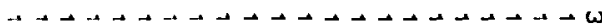

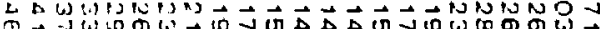

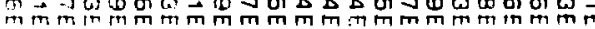

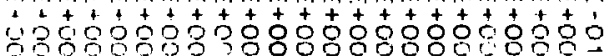

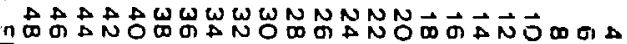

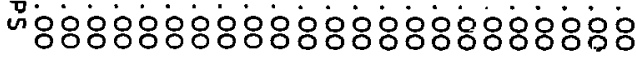

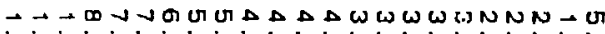

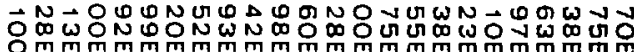

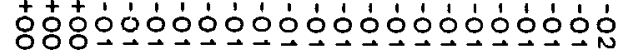
-

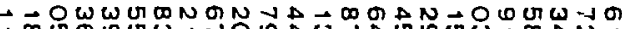

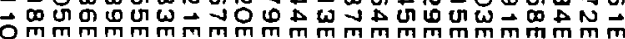

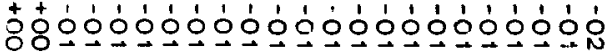
ம $\rightarrow \omega$ o $0 \omega$ os ONm

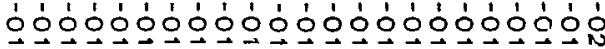

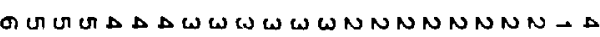

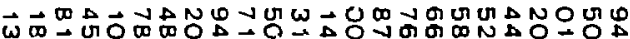

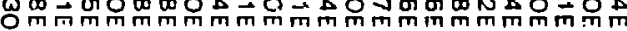

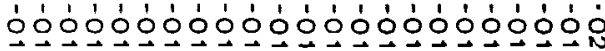
$\omega \omega \omega \omega \omega \omega M N N N N N N N$ N

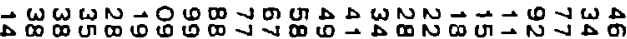

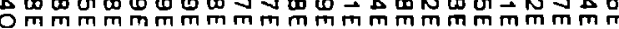

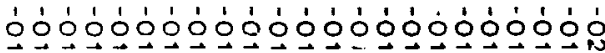

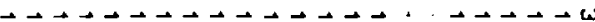

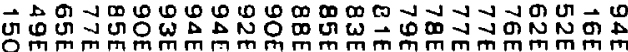

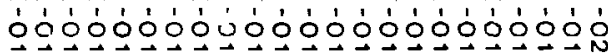

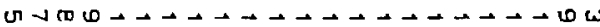

U U U

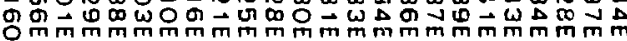

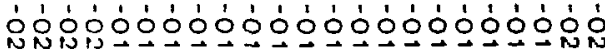
NW

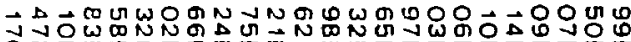
O m m m m m m m m m m m m m m m m m m

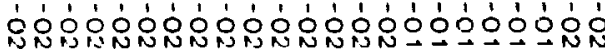
$\therefore$ MNWW

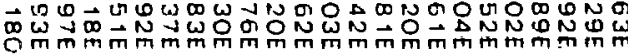

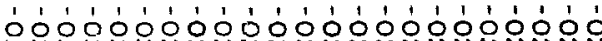
$\rightarrow+.+N$. + N O-

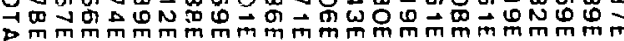

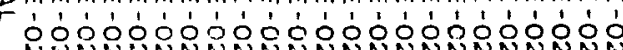

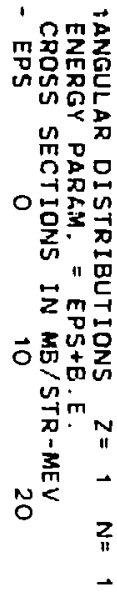

ڤ

o

등

I

oี

\&

8 
员

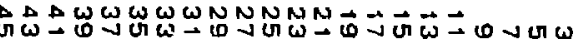
8888888888888898888888

()

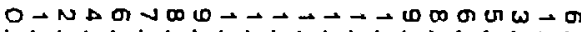
$\rightarrow \infty$ o $\rightarrow$ un

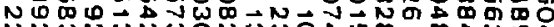

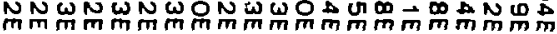

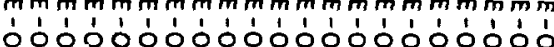

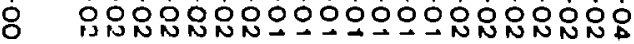

- 0000000000000000000000

8

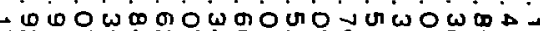

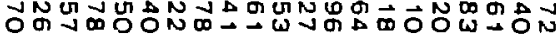
읶 $\mathrm{mm}$ mm mmmmm

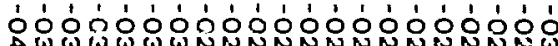

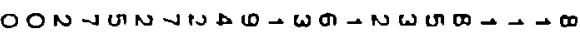

U 0 \% $A$ O

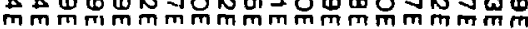

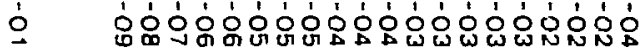

$\rightarrow$

皿

w

o

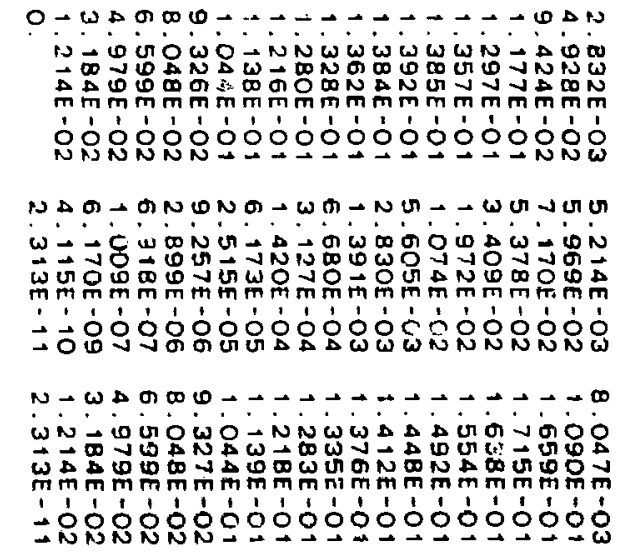

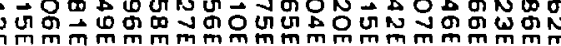

'́óóóóóóóóóóóóóóóóó

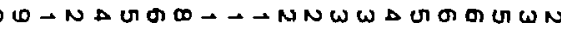

更

ins $n=0+\pi \overrightarrow{0}$

mำง

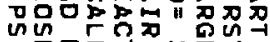

mogn

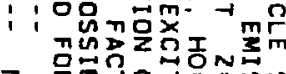

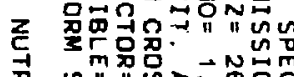

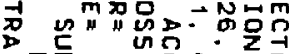

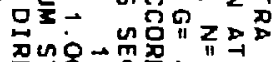

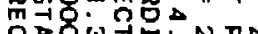

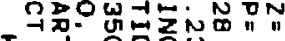

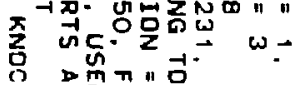

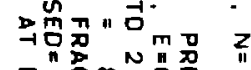

จ"

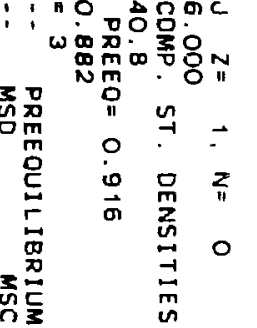

$\vdots:$

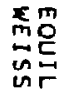

$\therefore$

疋

몸

竞

$\underset{\substack{\mathbf{m} \\ \mathbf{m}}}{\mathbf{m}}$

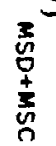




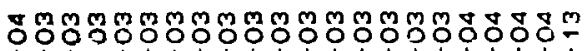

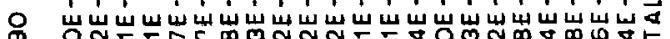

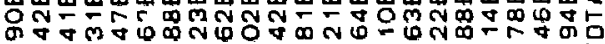

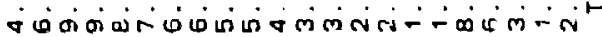

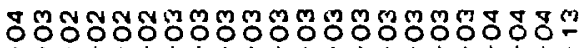

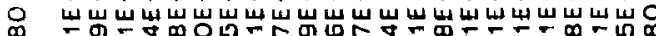

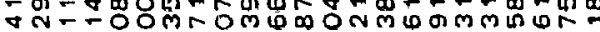

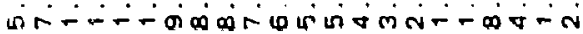

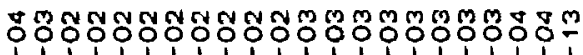

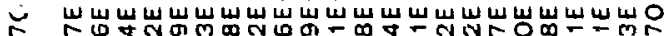

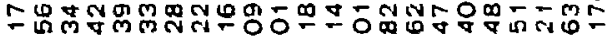

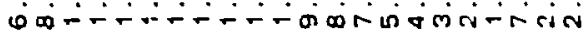

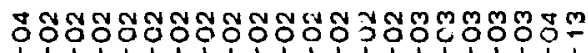

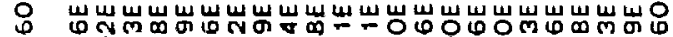

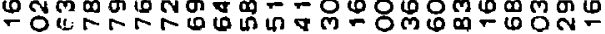

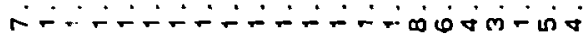

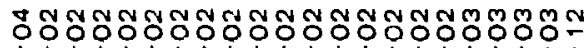

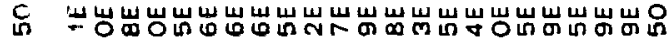

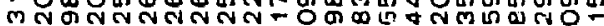

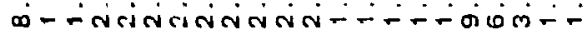

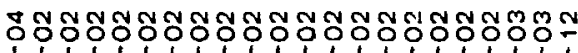

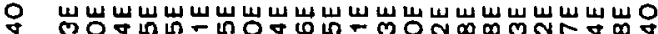

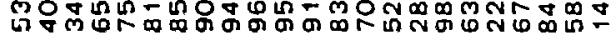

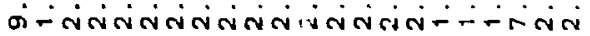

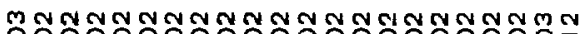

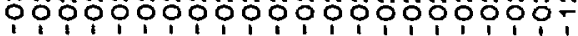

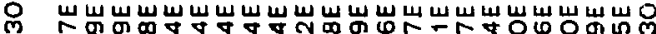

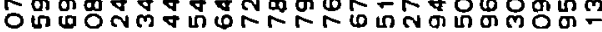

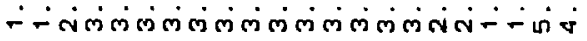

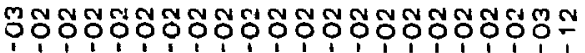

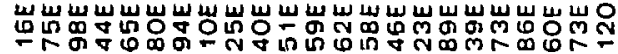

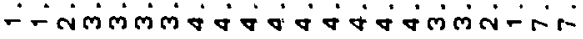

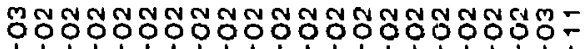

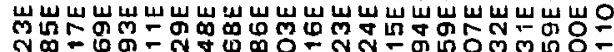
$\therefore$ लिलिव वें

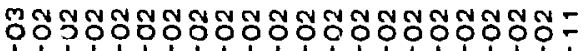

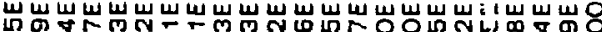

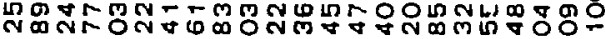

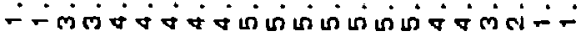

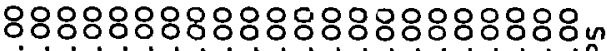

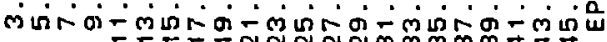

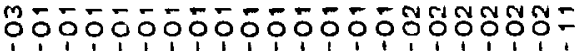

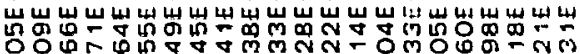

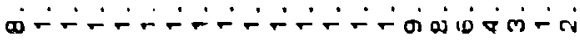

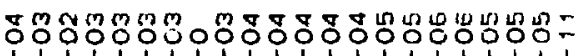

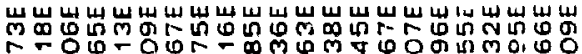

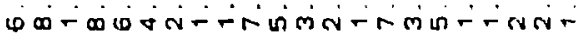

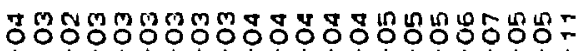

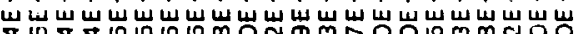

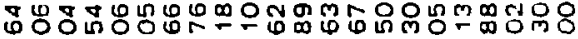

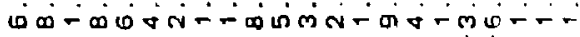

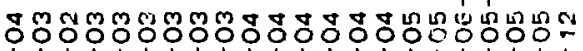

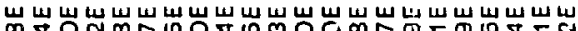

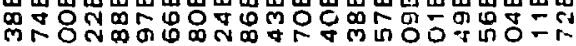

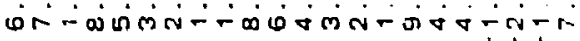

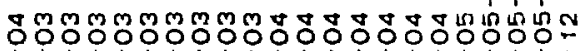

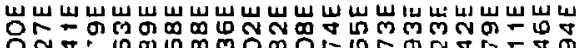

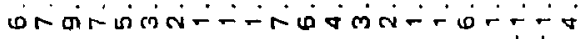

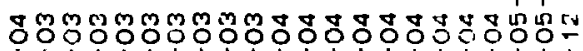
س

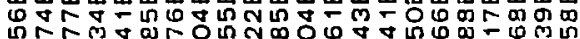

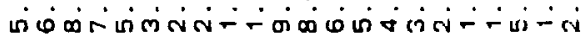

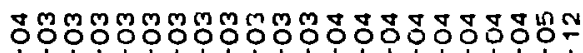

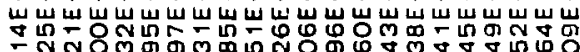
ம்

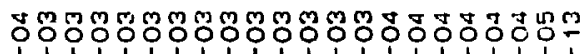

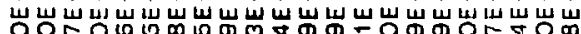

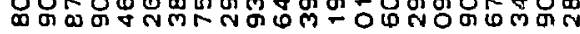

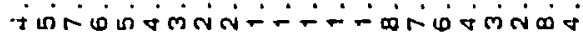

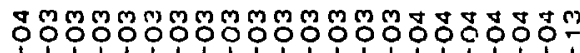

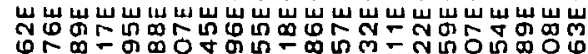

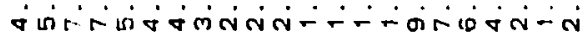

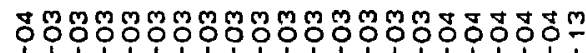

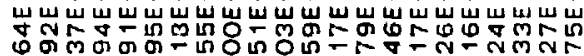
पंம் 8888888888888888988880

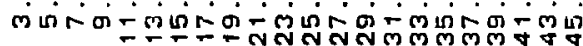


总

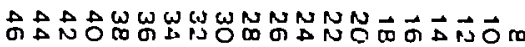
88888888888888888988

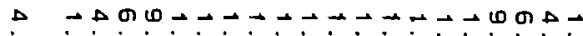

$\omega$ n v

O $\omega \omega \infty$ NONOOA N N

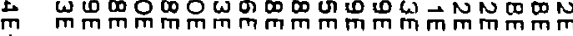

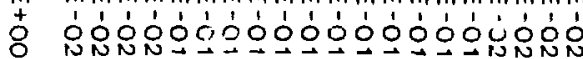

- 00000000000000000000

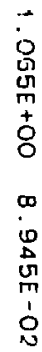

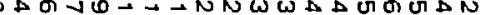

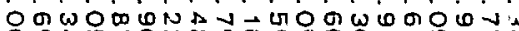

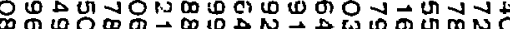

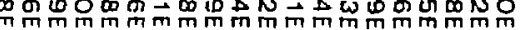
óóóóóóóóóóóóóo

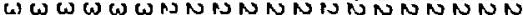

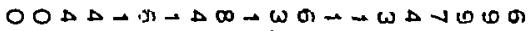
Oo

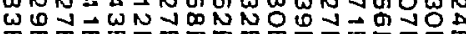
1 1 1 1 1 1 1 1 1 1

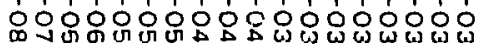

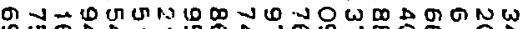

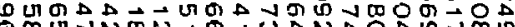

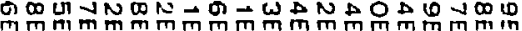
'́óóóóóóóóóóóóó $0 \infty 01+\omega$ $\omega O N$ J 至

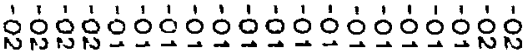

$\rightarrow-U A N-N G+N \pi \rightarrow N \Delta-N-N G \Delta N$ g

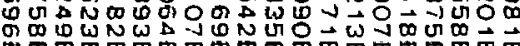

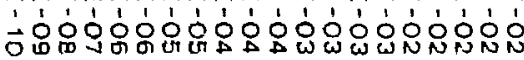

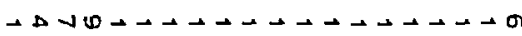

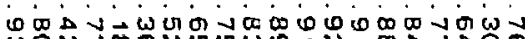

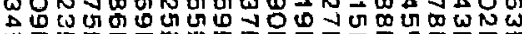

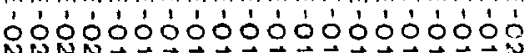

$\ln \sin 0+\pi \overrightarrow{0}$

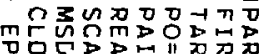
in

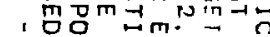

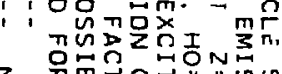

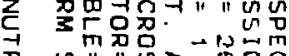

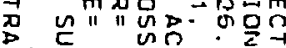
口芆음

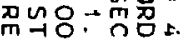

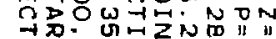

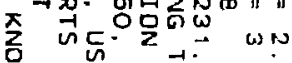

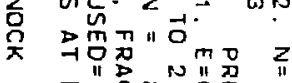
궁

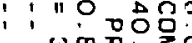
$\omega \operatorname{mog}_{\text {min }}$ in N 


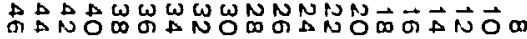
88888888888888888888

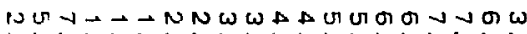

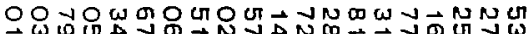

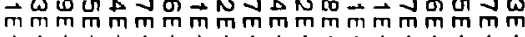
óóóóóóóóóóóóóó $\rightarrow 2 N N \omega$ o JU一

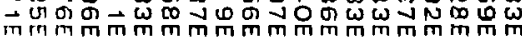
óoóóóóóóóóóóóóó

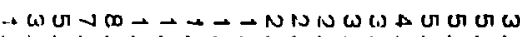

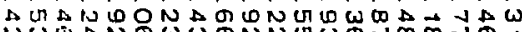

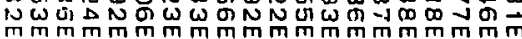

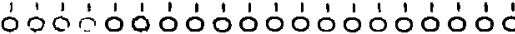

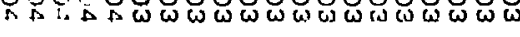
on $N$ r

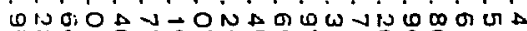
mm

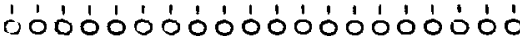

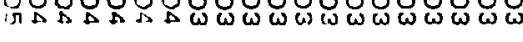
$\rightarrow 1 \rightarrow N \omega \triangleright \pi-1 \omega \rightarrow \rightarrow-1 N N \omega \triangle \operatorname{Mr} \omega$

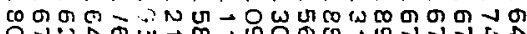

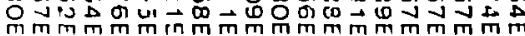
́́óóóóóóóóóóóóóó !

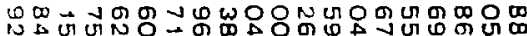
公药 óóóóóóóóćóó丶万ó

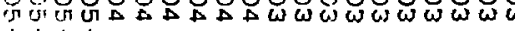
$\therefore$ is un $\Delta+N \omega \Delta \sigma-1+-N \omega \Delta \sigma a \Delta$

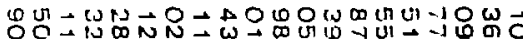

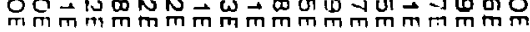

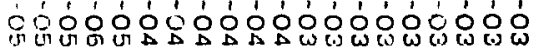

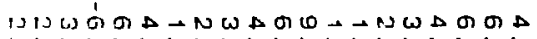
-1

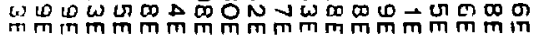

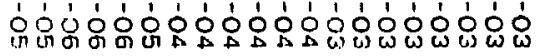

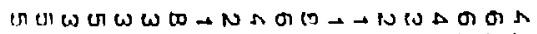

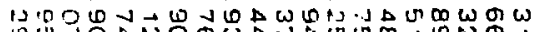

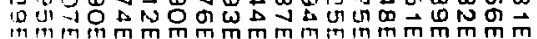

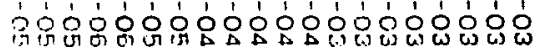

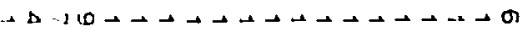

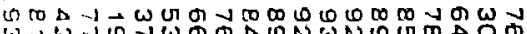

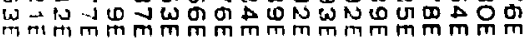

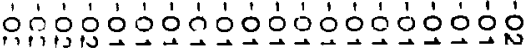

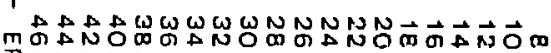

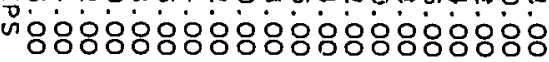

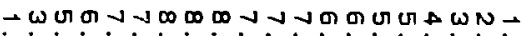

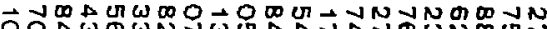

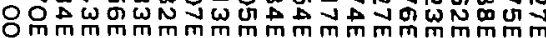

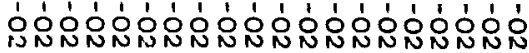

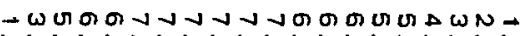

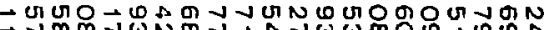

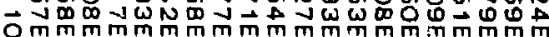

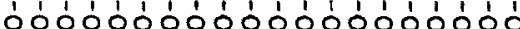

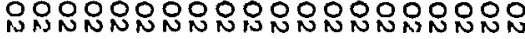

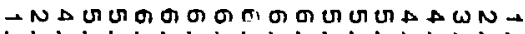

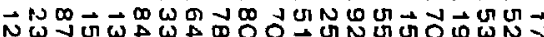

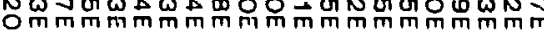

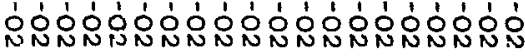

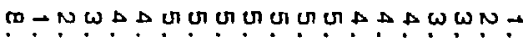
ம

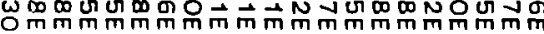

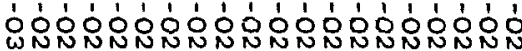
$\triangle \rightarrow-N N \omega \omega W A A \Delta A \Delta \omega \omega W \omega N \rightarrow$

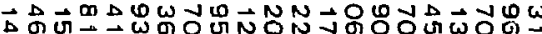

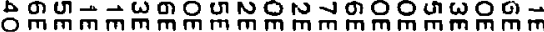

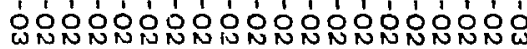
- Uח- - NNNNNWWEWNNNN - V $\rightarrow$ 은

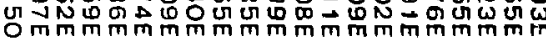

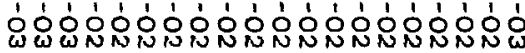

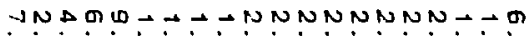
$\rightarrow \omega \omega A D A N D O D O A N N N N \rightarrow O N W \sigma$

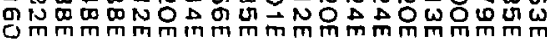
óóóóóóóóóóóóóóóó B LELENNNNNNNNNANNNNL

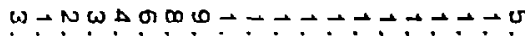

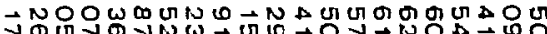

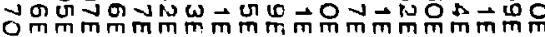

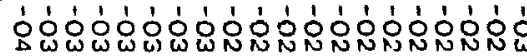

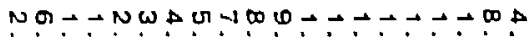

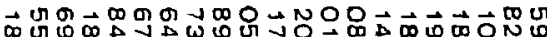

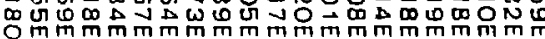

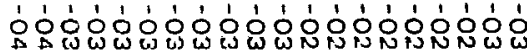

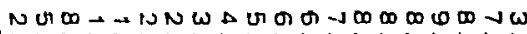
乙 w 元 $>\mathrm{m} m \mathrm{~mm} \mathrm{~mm} \mathrm{mmmm} \mathrm{mm} m \mathrm{~mm}$

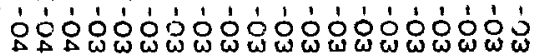

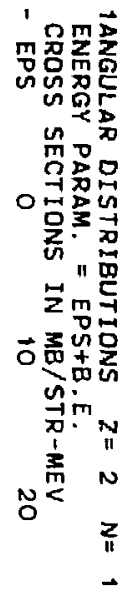

ڤ

ô

ㄴำ

용 
苍 1 -S $=5.69$ MEV

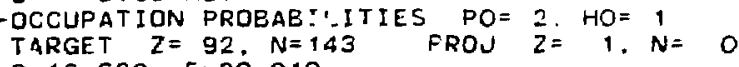
$G=16,620, E=20.040$

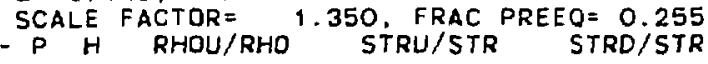

$\begin{array}{rrrll}1 & 0 & 1.000 E+O O & 0 . & \\ 2 & 1 & 8.741 E-O 1 & 8.741 E-01 & 0.741 E-01 \\ 3 & 2 & 7.182 E-01 & 6.998 E-01 & 6.662 E-01 \\ 4 & 3 & 5.258 E-01 & 5.045 E-01 & 4.229 E-01 \\ 5 & 4 & 3.387 E-01 & 3.060 E-01 & 2.183 E-01 \\ 6 & 5 & 1.997 E-01 & 1.611 E-01 & 9.085 E-02 \\ 7 & E & 1.091 E-01 & 7.821 E-02 & 3.004 E-02 \\ B & 7 & 5.519 E-02 & 3.832 E-02 & 7.700 E-03 \\ 9 & 8 & 2.570 E-02 & 2.048 E-02 & 1.477 E-03 \\ 10 & 9 & 1.087 E-02 & 1.202 E-02 & 0.02\end{array}$




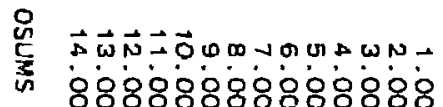

- 00000000000000

- 00000000000000

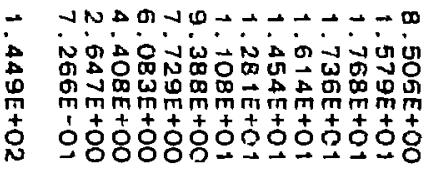

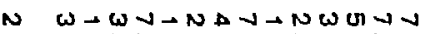

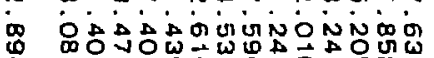
至 † ó

$\rightarrow \infty-\infty N N \rightarrow \infty \omega \rightarrow$ ONN

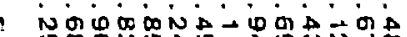

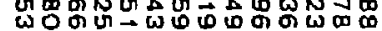

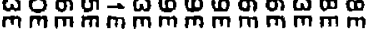

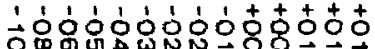

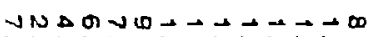

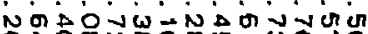

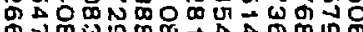
mm

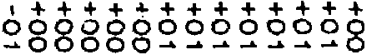

$\omega-\omega-N>\infty-\omega$ ON On os a

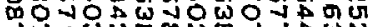

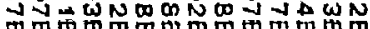
ó

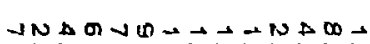
Non $\rightarrow 00$ is

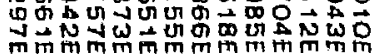

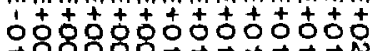

' $2 \leq n \pi 007$

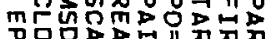

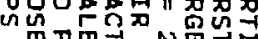

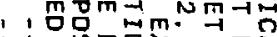

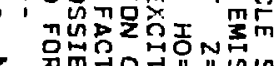
2 쪼엉에 " 敒" 口产。

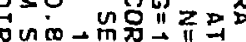

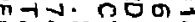

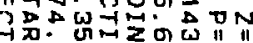
$\pi^{-1}-1000$

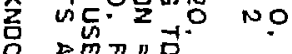
十0 $m$ o " $"$ 员 N"

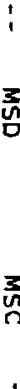
Nis

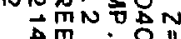

3

ป⿱一兀⿵冂⿱一口㇈丶 监 01 $0 \stackrel{-1}{0}=$

留 品 出 窝 "

$\frac{z}{y}$ 


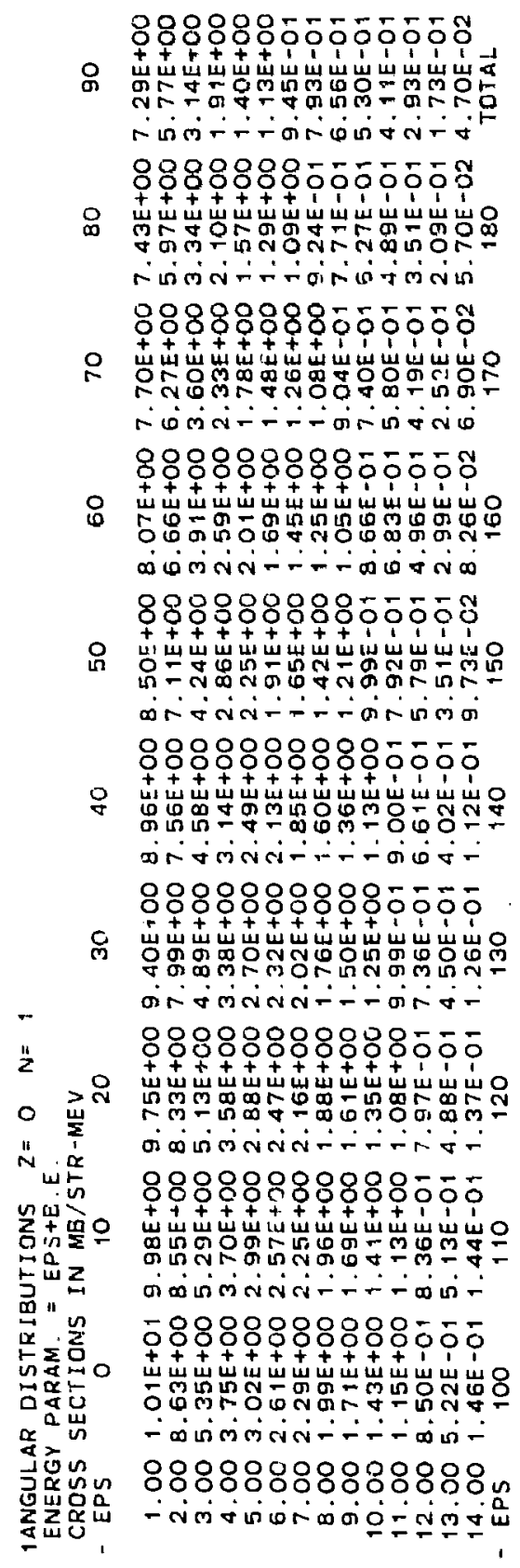

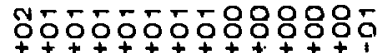

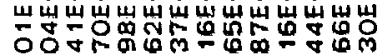
वरrन-raindir

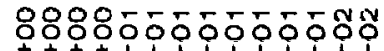

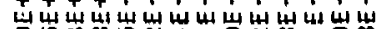

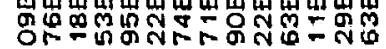

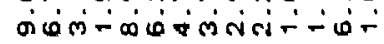
$8888000 \overline{1} 0 \overline{0} 0000$ $+++t 119$

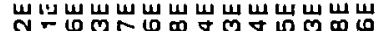

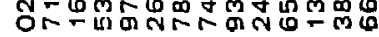

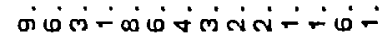

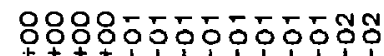

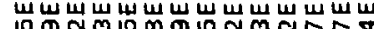
แू⿱

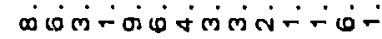

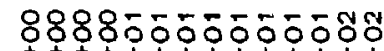
$++\ldots+1,1$,

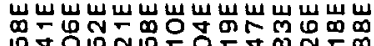
ம்

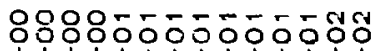

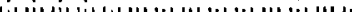

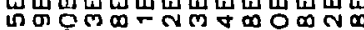
กิ कம

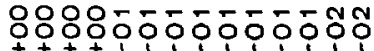

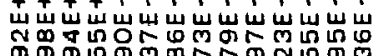

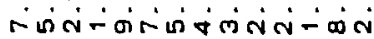

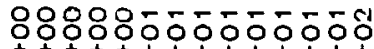

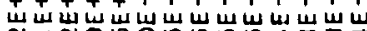

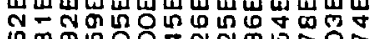

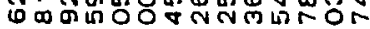
$\therefore$ ம்- -

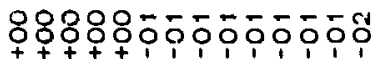

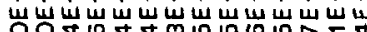

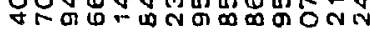

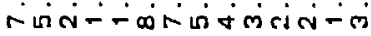

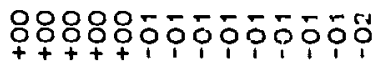

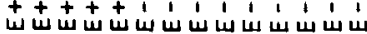

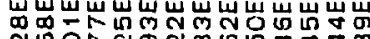

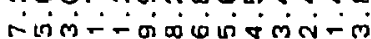

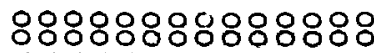

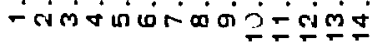


IPART ICLE SPECTRA $Z=1, N=0$

FIRST EMISSION AT $P=2$

TARGET $Z=92, N=143$ PROU $Z=1 . N=0$

$P O=2, H O=1, G=16,620, E=20.040$

PAIR EXCIT ACCORDING TO 2 CDMP. ST. DENSITIES

REACTION CROSS SECTION $=743.2$

SCALE FACTDR = 1.350 , FRAC PREEO $=0.255$

MSD POSSIBLE $=0.874$, USED $=0.214$

CLOSED FORM SUM STARTS AT $P=2$

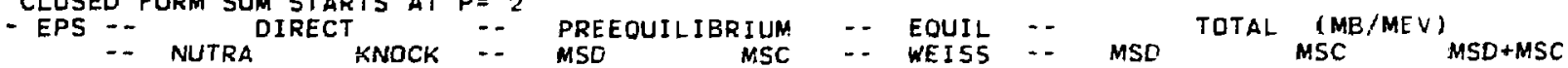

\begin{tabular}{|c|c|c|c|c|c|c|c|c|}
\hline $\begin{array}{r}9.00 \\
10.00 \\
11.00 \\
12.00 \\
13.00 \\
14.00 \\
15.00\end{array}$ & $\begin{array}{l}0 . \\
0 . \\
0 . \\
0 . \\
0 . \\
0 . \\
0 .\end{array}$ & $\begin{array}{l}1.570 E-O 3 \\
7.964 E-O 3 \\
1.586 E-O 2 \\
2.300 E-O 2 \\
2.632 E-C 2 \\
2.200 E-O 2 \\
0 .\end{array}$ & $\begin{array}{l}1.182 E-01 \\
5.501 E-01 \\
1.024 E+00 \\
1.410 E+00 \\
1.562 E+00 \\
1.295 E+00 \\
3.528 E-01\end{array}$ & $\begin{array}{l}3.811 E-03 \\
1.206 E-02 \\
1.513 E-02 \\
1.399 E-02 \\
1.055 E-02 \\
6.371 E-03 \\
1.529 E-03\end{array}$ & $\begin{array}{l}9.428 E-05 \\
7.402 E-05 \\
1.967 E-05 \\
3.136 E-06 \\
3.026 E-07 \\
1.443 E-08 \\
1.443 E-10\end{array}$ & $\begin{array}{l}1.197 E-01 \\
5.581 E-01 \\
1.039 E+00 \\
1.433 E+00 \\
1.58 B E+00 \\
1.317 E+00 \\
3.62 B E-01\end{array}$ & $\begin{array}{l}3.905 E-03 \\
1.214 E-02 \\
1.515 E-02 \\
1.399 E-02 \\
1.055 E-02 \\
6.371 E-03 \\
1.529 E-0 E\end{array}$ & $\begin{array}{l}1.237 E-01 \\
5.702 E-01 \\
1.055 E+C O \\
1.447 E+00 \\
1.599 E+00 \\
1.323 E+00 \\
3.643 E-01\end{array}$ \\
\hline UUMS & 0. & $9.071 \mathrm{E}-02$ & 6. $322 E+\infty 0$ & $6.345 E-02$ & $1.914 \mathrm{E}-04$ & & & \\
\hline
\end{tabular}




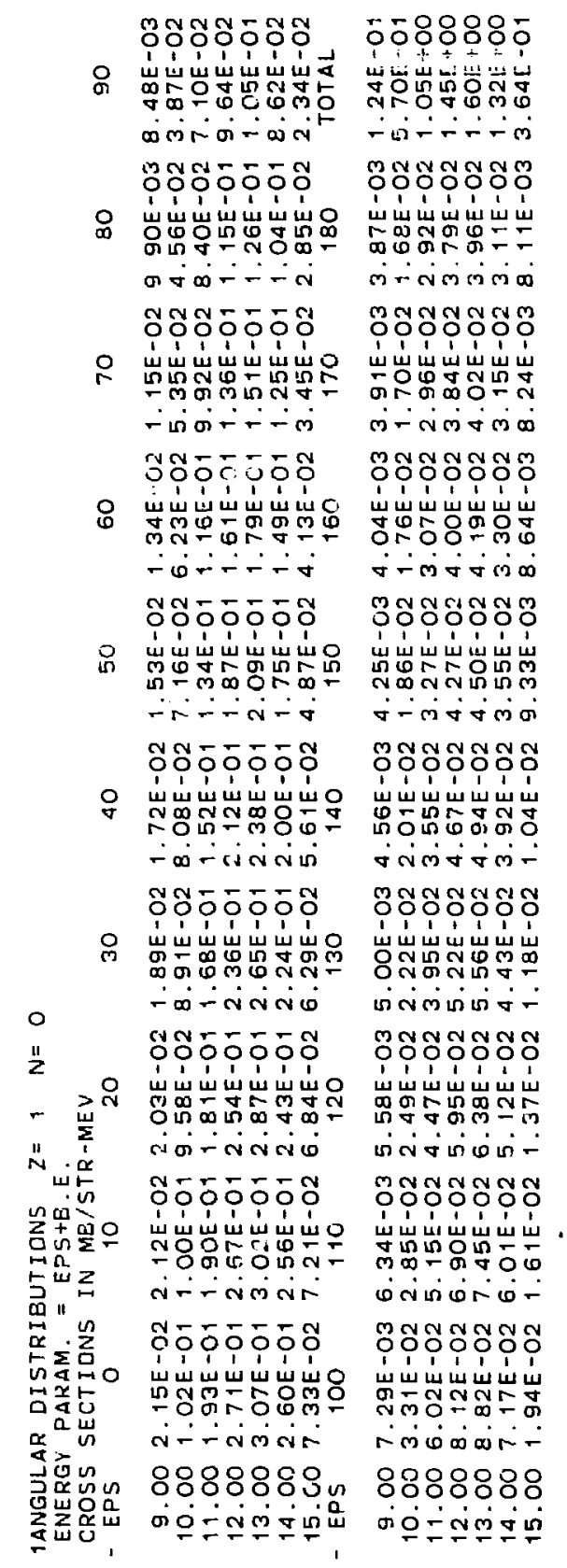


IPART ICLE SPECTRA $Z=2, N=2$

FIRST EMISSION AT $P=4^{\circ}, N=2$

TARGET $Z=92, N=143$ PROJ $Z=1, N=0$

$P O=2, H=1, G=16,620, E=20,040$

PAIR EXCIT ACCORDING TO 2 COMP ST DENSITIES

REACTION CROSS SECTION $=743.2$. ST. DENSITIES

SCALE FACTOR $=1.350$, FRAC PREEQ $=0.255$

HSD POSSIBLE $=0.874$ USED $=0.214$

CLOSED FORM SUM STARTS AT PI

- NUTRA

KNOCK -- $\quad$ MSD

MSD QUILIBRIUM

MSC $\quad-\quad$ EQUIL

MSD

TOTAL (MB/MEV)

$M S D+M S C$

$\begin{array}{llllllllll}21.00 & 5.665 E-O 2 & 8.147 E-O 2 & 1.319 E-O 4 & 6.297 E-05 & 2.667 E-O 5 & 1.383 E-01 & 8.964 E-05 & 1.383 E-01 \\ 22.00 & 8.886 E-02 & 1.555 E-01 & 1.570 E-O 4 & 5.829 E-05 & 6.133 E-06 & 2.445 E-01 & 6.442 E-05 & 2.446 E-01 \\ 23.00 & 7.333 E-02 & 1.553 E-01 & 1.055 E-04 & 3.221 E-05 & 6.527 E-07 & 2.287 E-01 & 3.286 E-05 & 2.2 B 8 E-01 \\ 24.00 & 2.867 E-02 & 0 . & 3.577 E-05 & 9.743 E-06 & 3.202 E-08 & 2.870 E-02 & 9.775 E-06 & 2.871 E-02\end{array}$

OSUMS

3. $577 \mathrm{E}-05$

9. $743 \mathrm{E}-06$

0

4. 302E-O4

1. $632 \mathrm{E}-\mathrm{O} 4$

3. $543 \mathrm{E}+\mathrm{O} 2$ 


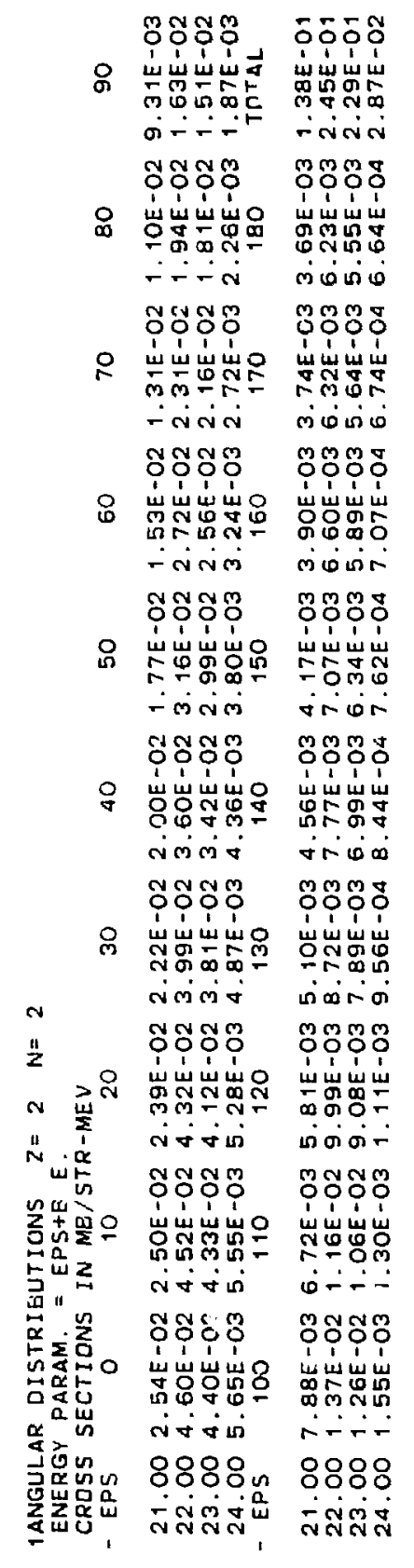




\section{APPENDIX D}

NEW FEATURES IN PRECO-D2

1. Pairing energies are read in separately for the preequilibrium and equilibrium phases of the reaction. (No pairing energies were read in in PRECO-D.)

2. Different effective well depths for states with $h=1$ and $h=2$ may be read in. (The well depth was always 38 $\mathrm{MeV}$ in PRECO-D, )

3. Default values are supplied for $p_{0}, h_{0}, g_{0}$ and $k$.

4. The composite nucleus-formation cross section and the inverse-reaction cross sections may be generated internally using a parametric approximation to the opticalmodel cross sectior.s. See Section VI.7 for the optical potentials which are approximated.

5. For nucleon transfer reactions, the residual configurations in which transfer produces passive particles and/or holes are not explicitly considered, because they are already counted in the general-state density formula.

6. For knockout and inelastic-scattering reactions involving cluster degrees of freedom, the single-clusterstate densities have been reduced. In PRECO-D they were derived by considering the number of ways in which the constituent nucleons could be put into correlated singlenucleon states. The desired quantity is, instead, given as [\# cluster states]/[MeV carried by cluster], which adds 
a factor of $1 / A_{\text {cluster }}$. In addition, the quantity of interest is really the number of clusters that can be. accommodated per MeV of cluster energy, not the number of ways of putting the constituent nucleons in a given cluster into the correlated single-nucleon states. This adds a factor of $1 / 2$ for deuterons, tritons and ${ }^{3}$ He ions. See section II. 2 for the current single-cluster-state densities.

7. The closed-form reaction equations have been corrected to guarantee conservation of strength.

8. Either $\varepsilon$ or $\varepsilon+B_{b}$ may be used as the energy parameter for calculating angular distributions. (In PRECO-D the energy parameter was always taken to be $\varepsilon$. )

9. Either $Q_{b}(p)$ or $Q_{b}^{(G)}(p)$ may be used for handling proton/neutron distinguishability in calculating particle emission rates. (In PRECO-D only $Q_{b}(p)$ was available.)

10. For each particle spectrum, output for a given $\varepsilon$ is suppressed if all of the component cross sections are zero. 